\title{
On the Motion of a Compressible Gravity Water Wave with Vorticity
}

\author{
Chenyun Luo
}

\begin{abstract}
We prove a priori estimates for the compressible Euler equations modeling the motion of a liquid with moving physical vacuum boundary with unbounded initial domain. The liquid is under influence of gravity but without surface tension. Our fluid is not assumed to be irrotational. But the physical sign condition needs to be assumed on the free boundary. We generalize the method used in [20] to prove the energy estimates in an unbounded domain up to arbitrary order. In addition to that, the a priori energy estimates are in fact uniform in the sound speed $\kappa$. As a consequence, we obtain the convergence of solutions of compressible Euler equations with a free boundary to solutions of the incompressible equations, generalizing the result of $[20$ to when you have an unbounded domain. On the other hand, we prove that there are initial data satisfying the compatibility condition in some weighted Sobolev spaces, and this will propagate within a short time interval, which is essential for proving long time existence for slightly compressible irrotational water waves.
\end{abstract}

\section{Contents}

1 Introduction

2 The Lagrangian coordinates

3 Basic estimates on the domain with free boundary

4 The wave equation

5 Energy estimates for Euler equations with free boundary

6 Incompressible limit

7 The initial data and the physical condition

8 The weighted a priori estimates for the Euler equations

A Appendix

\section{Introduction}

We consider the compressible water wave problem in $\mathbb{R}^{n}, n=2,3$. We use the notation $\mathcal{D}_{t}:=\left\{\left(x^{\prime}, x_{n}\right) \in\right.$ $\left.\mathbb{R}^{n}: x_{n} \leq \Sigma\left(t, x^{\prime}\right)\right\}$ to represent the domain occupied by the fluid at each fixed time $t$, whose boundary $\partial \mathcal{D}_{t}=\left\{\left(x^{\prime}, x_{n}\right): x_{n}=\Sigma\left(t, x^{\prime}\right)\right\}$ moves with the velocity of the fluid. Under this setting, the motion of the fluid is described by the Euler equations

$$
\left\{\begin{array}{l}
D_{t} v:=\partial_{t} v+\nabla_{v} v=-\frac{1}{\rho} \partial p-g \mathfrak{e}_{n}, \quad x \in \mathcal{D} \\
D_{t} \rho+\rho \operatorname{div} v=0 \quad x \in \mathcal{D}
\end{array}\right.
$$


with the initial and boundary condition

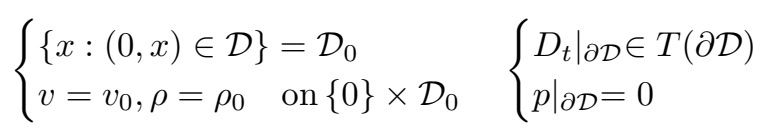

where $\mathcal{D}:=\cup_{0 \leq t \leq T}\{t\} \times \mathcal{D}_{t}, g>0$ is the gravity constant and $\rho$ denotes the density of the fluid and the equation of the state is given by

$$
p=p(\rho), \quad p^{\prime}(\rho)>0, \quad \text { for } \quad \rho \geq \bar{\rho}_{0},
$$

where $\bar{\rho}_{0}:=\left.\rho\right|_{\partial \mathcal{D}}>0$ is a constant (for simplicity, we set $\bar{\rho}_{0}=1$ ), which is in the case of a liquid.

We prove the energy estimates for the local (in time) solutions of system (1.1)-(1.2), taking prescribed initial data, such that for every fixed time $t \in[0, T],|v(t, x)| \rightarrow 0,\left|v_{t}(t, x)\right| \rightarrow 0$, and $\Sigma\left(t, x^{\prime}\right) \rightarrow\left\{\left(x^{\prime}, 0\right)\right.$ : $\left.x^{\prime} \in \mathbb{R}^{n-1}\right\}$ as $|x| \rightarrow \infty$. In fact, we are able to show that there exist initial data satisfying the compatibility condition (1.6) in some weighted Sobolev spaces with weight $w(x)=\left(1+|x|^{2}\right)^{\mu}, \mu \geq 2$. This implies that our data is at least of $O\left(|x|^{-2}\right)$ as $|x| \rightarrow \infty$.

We introduce the enthalpy $h$ to be a function of the density, i.e., $h(\rho)=\int_{1}^{\rho} p^{\prime}(\lambda) \lambda^{-1} d \lambda$. Since $\rho \geq \bar{\rho}_{0}=1$ can then be thought as a function of $h$, we define $e(h)=\log \rho(h)$. Under these new variables, (1.1)-(1.2) can be re-expressed as

$$
\left\{\begin{array}{l}
D_{t} v=-\partial h-g \mathfrak{e}_{n}, \quad \text { in } \mathcal{D} \\
\operatorname{div} v=-D_{t} e(h)=-e^{\prime}(h) D_{t} h . \quad \text { in } \mathcal{D}
\end{array}\right.
$$

Together with initial and boundary conditions

$$
\left\{\begin{array} { l } 
{ \{ x : ( 0 , x ) \in \mathcal { D } \} = \mathcal { D } _ { 0 } , } \\
{ v = v _ { 0 } , h = h _ { 0 } \quad \text { on } \{ 0 \} \times \mathcal { D } _ { 0 } . }
\end{array} \quad \left\{\begin{array}{l}
\left.D_{t}\right|_{\partial \mathcal{D}} \in T(\partial \mathcal{D}), \\
h=0 \text { on } \partial \mathcal{D} .
\end{array}\right.\right.
$$

(1.3) looks exactly like the incompressible Euler equations, where $h$ takes the position of $p$ and $\operatorname{div} v$ is no longer 0 but determined by $h$. In addition, we take the gravity constant $g=1$. On the other hand, we would like to impose the following natural conditions on $e(h)$ : For each fixed $r \geq 1$, there exists a constant $c_{0}$ such that

$$
\left|e^{(k)}(h)\right| \leq c_{0}, \quad \text { and }\left|e^{(k)}(h)\right| \leq c_{0}\left|e^{\prime}(h)\right|^{k} \leq c_{0}\left|e^{\prime}(h)\right|, \quad \text { if } k \leq r+1 .
$$

In order for the initial boundary problem (1.3)-(1.4) to be solvable the initial data has to satisfy certain compatibility conditions at the boundary. By the second equation in (1.1),(1.2) implies that $\left.\operatorname{div} v\right|_{\partial \mathcal{D}}=0$. We must therefore have $\left.h_{0}\right|_{\partial \mathcal{D}_{0}}=0$ and $\left.\operatorname{div} v_{0}\right|_{\partial \mathcal{D}_{0}}=0$, which is the zero-th compatibility condition. Furthermore, $m$-th order compatibility condition can be expressed as

$$
\left.\left(\partial_{t}+v^{k} \partial_{k}\right)^{j} h\right|_{\{0\} \times \partial \mathcal{D}_{0}}=0 \quad j=0, \cdots, m .
$$

In [20], we have proved that for each fixed $m$, there exists initial data satisfying $m$-th order compatibility condition if the sound speed $c(t, x)$ is sufficiently large. In addition, the energies $E_{r}$, defined as (1.12), are bounded uniformly at time 0 , regardless of the sound speed.

Let $N$ be the exterior unit normal to the free surface $\partial \mathcal{D}_{t}$. We will prove a priori bounds for (1.3)-(1.4) in Sobolev spaces under the assumption

$$
\nabla_{N} h \leq-\epsilon<0 \quad \text { on } \partial \mathcal{D}_{t}
$$

where $\nabla_{N}=N^{i} \partial_{i}$ and $\epsilon>0$ is a constant. (1.7) is a natural physical condition. It says that the pressure and hence the density is larger in the interior than at the boundary. The system (1.1)-(1.2) is ill-posed in absence of (1.7), an easy counter-example can be found in [5] and [10. Furthermore, if the fluid is assumed to be incompressible $(\operatorname{div} v=0)$ and irrotatonal ( $\operatorname{curl} v=0)$, (1.7) can in fact be proved via strong maximum principle. In addition, under the presence of the gravity, it can be shown that $-\nabla_{N} p$ can actually be bounded uniformly by some positive constants from below (see $\mathrm{Wu}[26,27]$ ). In fact, we show that the assumption 
is plausible by proving (1.7) in the case when the liquid is slightly compressible and irrotational (Section 7). Heuristically, in the Lagrangian coordinates (where $x:=x(t, y), \frac{d x(t, y)}{d t}=v(t, x(t, y)$ ), see Section 2), we have $x_{t t}=v_{t}$ and so

$$
-\nabla_{N} h=x_{t t} \cdot N+N \cdot e_{n}
$$

and because $v_{t}=x_{t t}$ decays to 0 at infinity, we conclude $-\nabla_{N} h \geq \epsilon>0$ for some $\epsilon>0$ pointwisely. We shall discuss more about this in Section 7 (see the remark after Theorem 17.4). But (1.7) needs to be assumed if the fluid is rotational and without surface tension.

Euler equations involving free-boundary has been studied intensively by many authors. The first break through in solving the well-posedness for the incompressible and irrotational water wave problem for general data came in the work of $\mathrm{Wu}$ [26, 27, who solved the problem in both two and three dimensions. For the general incompressible problem with nonvanishing curl Christodoulou and Lindblad [5] were the first to obtain the energy estimates assuming the physical sign condition. In addition, Zhang and Zhang [30] generalized Wu's work to incompressible water wave with nonvanishing curl. For the compressible problem, Lindblad [19] later proved local well-posedness for the general problem modeling the motion of a liquid in a bounded domain by Nash-Moser iteration, and this result was generalized to the case of an unbounded domain by Trakhinin [24. But these results do not contain a priori estimates for the solution due to the loss of regularity on the moving boundary.

Very recently, together with Lindblad, we obtained a new type of a priori energy estimates for the compressible Euler equations with free boundary in a bounded domain, which are uniform in the sound speed [20. This, in fact, leads to the convergence of solutions of compressible Euler equations with a free boundary to solutions of the incompressible equations in a bounded domain. In other words, we proved the so-called incompressibe limit problem for compressible free boundary Euler equations. It is worth mentioning here that the incompressible limit in $\mathbb{R}^{n}$ or $\mathbb{T}^{n}$ was established in [8, 17, 18, 21, 22, and there are further works (e.g., 1, 3, 4, 7, 9, 23, ) that treat the incompressible limit in domains with fixed boundary.

The goal of this paper is to generalize the above results to compressible water waves, i.e., the fluid domain becomes unbounded and diffeomorphic to the half space. To our knowledge, these results appear to be the first that concern a priori energy bounds for a compressible water wave. Furthermore, the incompressible limit allows one to approximate a slightly compressible water wave by an incompressible water wave, for which the long time existence is well-known, e.g., 12, 14, 15, 16, 28, 29. In addition, we show that the a priori energy estimates can also be generalized to weighted $L^{2}$-Sobolev spaces, which is an essential first step for proving long time existence also for compressible water waves.

\subsection{Energy conservation and higher order energies}

The boundary conditions $\left.p\right|_{\partial \mathcal{D}_{t}}=0$ and $\left.\rho\right|_{\partial \mathcal{D}_{t}}=1$ leads to that the zero-th order energy is conserved, i.e., let

$$
E_{0}(t)=\frac{1}{2} \int_{\mathcal{D}_{t}} \rho|v|^{2} d x+\int_{\mathcal{D}_{t}} \rho Q(\rho) d x+\int_{\mathcal{D}_{t} \cap\left\{x_{n}>0\right\}} x_{n} d x-\int_{\mathcal{D}_{t}^{c} \cap\left\{x_{n}<0\right\}} x_{n} d x+\int_{\mathcal{D}_{t}}(\rho-1) x_{n} d x
$$

where $Q(\rho)=\int_{1}^{\rho} p(\lambda) \lambda^{-2} d \lambda$. These integrals are bounded here because of the decay properties of our functions involved.

A direct computation yields

$$
\begin{array}{r}
\frac{d}{d t} E_{0}(t)=-\int_{\mathcal{D}_{t}}\left(\partial_{i} p\right) v^{i} d x-\int_{\mathcal{D}_{t}} \rho\left(\partial_{i} x_{n}\right) v^{i} d x+\int_{\mathcal{D}_{t}} p(\rho) D_{t} \rho \rho^{-1} d x+\int_{\partial \mathcal{D}_{t}} s \cdot v_{N} d S+\int_{\mathcal{D}_{t}}\left(\partial_{t} \rho\right) x_{n} d x \\
=\left(\int_{\mathcal{D}_{t}} p(\operatorname{div} v) d x+\int_{\mathcal{D}_{t}} p D_{t} \rho \rho^{-1} d x\right)+\left(\int_{\mathcal{D}_{t}}(v \cdot \partial \rho+\rho \operatorname{div} v) x_{n} d x+\int_{\mathcal{D}_{t}}\left(\partial_{t} \rho\right) x_{n} d x\right)=0 .
\end{array}
$$

The higher order energies $E_{r}(t)$ are defined in a similar fashion, but instead of using the regular inner product, we introduce a positive definite quadratic form $Q$ which, when restricted to the boundary, is the inner product of the tangential components, i.e., $Q(\alpha, \beta)=\Pi \alpha \cdot \Pi \beta$, where $\alpha$ and $\beta$ are $(0, r)$ tensors. To be more specific, we define

$$
Q(\alpha, \beta)=q^{i_{1} j_{1}} \cdots q^{i_{r} j_{r}} \alpha_{i_{1} \cdots i_{r}} \beta_{j_{1} \cdots j_{r}},
$$


where

$$
\begin{array}{r}
q^{i j}=\delta^{i j}-\eta(d)^{2} \mathcal{N}^{i} \mathcal{N}^{j} \\
d(x)=\operatorname{dist}\left(x, \partial \mathcal{D}_{t}\right), \\
\mathcal{N}^{i}=-\delta^{i j} \partial_{j} d
\end{array}
$$

Here $\eta$ is a smooth cut-off function satisfying $0 \leq \eta(d) \leq 1, \eta(d)=1$ when $d \leq \frac{d_{0}}{4}$ and $\eta(d)=0$ when $d>\frac{d_{0}}{2}$. $d_{0}$ is a fixed number that is smaller than the injective radius $l_{0}$, which is defined to be the largest number $l_{0}$ such that the map

$$
\partial \mathcal{D}_{t} \times\left(-l_{0}, l_{0}\right) \rightarrow\left\{x: \operatorname{dist}\left(x, \partial \mathcal{D}_{t}\right)<l_{0}\right\},
$$

given by

$$
(\bar{x}, l) \rightarrow x=\bar{x}+l \mathcal{N}(\bar{x}),
$$

is an injection.

The higher order energies we propose are

$$
E_{r}=\sum_{s+k=r} E_{s, k}+K_{r}+\sum_{j \leq r+1} W_{j}^{2}, \quad r \geq 2, \quad E_{r}^{*}=\sum_{r^{\prime} \leq r} E_{r^{\prime}},
$$

where

$$
\begin{aligned}
E_{s, k}(t)= & \frac{1}{2} \int_{\mathcal{D}_{t}} \rho \delta^{i j} Q\left(\partial^{s} D_{t}^{k} v_{i}, \partial^{s} D_{t}^{k} v_{j}\right) d x+\frac{1}{2} \int_{\mathcal{D}_{t}} \rho e^{\prime}(h) Q\left(\partial^{s} D_{t}^{k} h, \partial^{s} D_{t}^{k} h\right) d x \\
& +\frac{1}{2} \int_{\partial \mathcal{D}_{t}} \rho Q\left(\partial^{s} D_{t}^{k} h, \partial^{s} D_{t}^{k} h\right) \nu d S,
\end{aligned}
$$

where $\nu=\left(-\nabla_{N} h\right)^{-1}$ and

$$
\begin{aligned}
K_{r}(t) & =\int_{\mathcal{D}_{t}} \rho\left|\partial^{r-1} \operatorname{curl} v\right|^{2} d x, \\
W_{r}(t) & =\frac{1}{2}\left\|\sqrt{e^{\prime}(h)} D_{t}^{r} h\right\|_{L^{2}\left(\mathcal{D}_{t}\right)}+\frac{1}{2}\left\|\nabla D_{t}^{r-1} h\right\|_{L^{2}\left(\mathcal{D}_{t}\right)} .
\end{aligned}
$$

Here $W_{r}$ is the (higher order) energy for the wave equation

$$
D_{t}^{2} e(h)-\Delta h=\left(\partial_{i} v^{j}\right)\left(\partial_{j} v^{i}\right),
$$

which is obtained by commuting divergence through the first equation of (1.1) using

$$
\left[D_{t}, \partial_{i}\right]=-\left(\partial_{i} v^{j}\right) \partial_{j}
$$

The energies $E_{r}$ defined above in fact control all components of

$$
\begin{array}{r}
\|v\|_{r, 0}:=\sum_{k+s=r, k<r}\left\|\partial^{s} D_{t}^{k} v\right\|_{L^{2}\left(\mathcal{D}_{t}\right)}, \\
\|h\|_{r}:=\sum_{k+s=r, k<r}\left\|\partial^{s} D_{t}^{k} h\right\|_{L^{2}\left(\mathcal{D}_{t}\right)}+\left\|\sqrt{e^{\prime}(h)} D_{t}^{r} h\right\|_{L^{2}\left(\mathcal{D}_{t}\right)}, \\
\left\|D_{t} h\right\|_{r, 1}=\sum_{k+s=r, k<r-1}\left\|\partial^{s} D_{t}^{k+1} h\right\|_{L^{2}\left(\mathcal{D}_{t}\right)}+\left\|\sqrt{e^{\prime}(h)} \nabla D_{t}^{r} h\right\|_{L^{2}\left(\mathcal{D}_{t}\right)}+\left\|e^{\prime}(h) D_{t}^{r+1} h\right\|_{L^{2}\left(\mathcal{D}_{t}\right)}, \\
\langle\langle h\rangle\rangle_{r}:=\sum_{k+s=r}\left\|\partial^{s} D_{t}^{k} h\right\|_{L^{2}\left(\partial \mathcal{D}_{t}\right)},
\end{array}
$$


in the interior and on the boundary (section 5). Although $E_{r}$ only controls the tangential components, the fact that we also control the divergence $\sum_{j \leq r+1} W_{j}^{2}\left(\right.$ through $\left.\operatorname{div} v=-D_{t} e(h)\right)$ and the curl $K_{r}$ allows us to control all components. In fact, by a Hodge type decomposition

$$
|\partial v| \lesssim|\bar{\partial} v|+|\operatorname{div} v|+|\operatorname{curl} v|
$$

where the tangential derivatives are given by $\bar{\partial} h=\Pi \partial h$. In addition, if $\left|\nabla_{N} h\right| \geq \epsilon>0$ then the boundary term gives an estimate for the regularity of the boundary. In fact, one can show that if $q$ vanishes on the boundary then

$$
\Pi \partial^{r} q=\left(\bar{\partial}^{r-2} \theta\right) \nabla_{\mathcal{N}} q+O\left(\partial^{r-1} q\right)+O\left(\bar{\partial}^{r-3} \theta\right)
$$

where $\theta$ is the second fundamental form of the boundary and $\bar{\partial}$ stand for tangential derivatives, so

$$
\left\|\bar{\partial}^{r-2} \theta\right\|_{L^{2}\left(\partial \mathcal{D}_{t}\right)}^{2} \leq \frac{C}{\epsilon} E_{r}^{*}+C \sum_{r^{\prime} \leq r-1}\left\|\partial^{r^{\prime}} h\right\|_{L^{2}\left(\partial \mathcal{D}_{t}\right)}^{2} .
$$

Now, because of the estimates (1.18)-(1.20), using elliptic estimates (section 3) one can show that

$$
\begin{array}{r}
\|v\|_{r, 0}^{2}+\|h\|_{r}^{2} \leq C_{r}\left(K, M, c_{0}, E_{r-1}^{*}\right) E_{r}^{*}, \\
\left\|D_{t} h\right\|_{r, 1}^{2}+\langle\langle h\rangle\rangle_{r}^{2} \leq C_{r}\left(K, M, c_{0}, \frac{1}{\epsilon}, E_{r-1}^{*}\right) E_{r}^{*},
\end{array}
$$

for some continuous function $C_{r}$. In fact, we use many of such functions throughout this paper, but we shall not distinguish them unless otherwise specified, i.e., $C_{r}$ would always denote continuous functions depend on constants $K, M, c_{0}, \frac{1}{\epsilon}$ and the energies $E_{r-1}^{*}$.

\subsection{The main results}

Sections 4 45 are devoted to prove a priori energy estimates implying that the energies $E_{r}$ remain bounded as long as certain a priori assumptions are true. To be specific, we show

Theorem 1.1. Let $v, h$ be the solutions for (1.3)-(1.4) and $E_{r}$ be the energy defined as (1.12), then for each fixed integer $r \geq 1$

$$
\left|\frac{d E_{r}(t)}{d t}\right| \leq C_{r}\left(K, \frac{1}{\epsilon}, M, c_{0}, E_{r-1}^{*}\right) E_{r}^{*}(t)
$$

holds, where $C_{r}$ are continuous functions and $E_{r}^{*}=\sum_{s=0}^{r} E_{s}$, provided (1.5) a priori assumptions

$$
\begin{array}{rr}
|\theta|+\frac{1}{l_{0}} \leq K, & \text { on } \partial \mathcal{D}_{t} \\
-\nabla_{N} h \geq \epsilon>0, & \text { on } \partial \mathcal{D}_{t} \\
1 \leq|\rho| \leq M, & \text { in } \mathcal{D}_{t} \\
\left|\partial^{j} \operatorname{curl}_{i j} v\right| \leq M, & \text { in } \mathcal{D}_{t} \\
|\partial v|+|\partial h|+\left|\partial^{2} h\right|+\left|\partial D_{t} h\right| \leq M, & \text { in } \mathcal{D}_{t} \\
\left|e^{\prime}(h) D_{t} h\right|+\left|e^{\prime}(h) D_{t}^{2} h\right| \leq M . & \text { in } \mathcal{D}_{t}
\end{array}
$$

The bounds (1.24) gives us control of geometry of the free surface $\partial \mathcal{D}_{t}$. A bound for the second fundamental form $\theta$ gives a bound for the curvature of $\partial \mathcal{D}_{t}$, and a lower bound for the injectivity radius of the exponential map $l_{0}$ measures how far off the surface is from self-intersecting. In the case when $\mathcal{D}_{t}$ is unbounded, the uniform a priori bounds for $\left|D_{t} h\right|$ and $\left|D_{t}^{2} h\right|$ are weakened to the bounds (1.29), and we need them to hold uniformly to pass to the incompressible limit.

Remark. $r$ will be used to denote an integer throughout this manuscript. In particular, we will not use fractional Sobolev norms. 
Remark. The assumption (1.29) can, in fact, be relaxed to $\left|\sqrt{e^{\prime}(h)} D_{t} h\right|+\left|\sqrt{e^{\prime}(h)} D_{t}^{2} h\right| \leq M$. However, we will no longer be able to pass (1.23) to the incompressible limit.

In Section 6, We show that the energy bounds (1.23) remain valid uniformly as the sound speed goes to infinity. For physical reasons, the sound speed is defined by $c(t, x)=\sqrt{p^{\prime}(\rho)}$. In this paper, the sound speed $\kappa$ is defined by viewing $\left\{p_{\kappa}(\rho)\right\}$ as a family parametrized by $\kappa \in \mathbb{R}^{+}$, such that for each $\kappa$ we have $\kappa:=\left.p_{\kappa}^{\prime}(\rho)\right|_{\rho=1}$. We consider the compressible Euler equations depend on $\kappa$ :

$$
\left\{\begin{array}{l}
D_{t} v_{\kappa}=-\partial h_{\kappa}-\mathfrak{e}_{n}, \\
\operatorname{div} v_{\kappa}=-D_{t} e_{\kappa}(h) .
\end{array}\right.
$$

Here, we further assume that $e_{\kappa}(h)$ satisfies:

$$
e_{\kappa}(h) \rightarrow 0, \quad \text { as } \kappa \rightarrow \infty,
$$

and for each fixed $r \geq 1$, there exists a constant $c_{0}$ such that

$$
\left|e_{\kappa}^{(k)}(h)\right| \leq c_{0}, \quad \text { and }\left|e_{\kappa}^{(k)}(h)\right| \leq c_{0}\left|e_{\kappa}^{\prime}(h)\right|^{k} \leq c_{0}\left|e_{\kappa}^{\prime}(h)\right|, \quad \text { if } k \leq r+1 .
$$

Under this setting, we show

Theorem 1.2. Let $\left(v_{\kappa}, h_{\kappa}\right)$ solves (1.30). Let $\widetilde{E}_{r}$ be defined as $\widetilde{E}_{r}=\sum_{s+k=r} E_{s, k}+K_{r}+\sum_{j \leq r+1} \widetilde{W}_{j}$, where

$$
\widetilde{W}_{j}=\frac{1}{2}\left\|e_{\kappa}^{\prime}(h) D_{t}^{j} h_{\kappa}\right\|_{L^{2}(\Omega)}+\frac{1}{2}\left\|\sqrt{e_{\kappa}^{\prime}(h)} \nabla D_{t}^{j-1} h_{\kappa}\right\|_{L^{2}(\Omega)} .
$$

If, in addition, the physical sign condition holds, i.e.,

$$
-\nabla_{N} h_{\kappa} \geq \epsilon>0
$$

then there exists $T>0$, independent of $\kappa$, such that for any smooth solutions of (1.30) for $0 \leq t \leq T$ satisfies

$$
\widetilde{E}_{r, \kappa}^{*}(t) \leq 2 \widetilde{E}_{r, \kappa}^{*}(0), \quad \text { whenever } r>n / 2+3 / 2
$$

and this estimate can be carried over to the case when $\kappa=\infty$, i.e., the energy estimates for the incompressible Euler equations.

Theorem 1.2 is a direct consequence of the a priori energy bounds (1.23) are uniform in $\kappa$ via Gronwall's lemma. Moreover, (1.23) remains valid since that our estimates do not depend on the lower bound of $e_{\kappa}^{(k)}(h)$, which goes to 0 as $\kappa \rightarrow \infty$, and the elliptic estimates (1.21)-(1.22) can be carried to the incompressible case apart from the term $\left\|\partial D_{t}^{k} h\right\|_{L^{2}\left(\mathcal{D}_{t}\right)}, 0 \leq k \leq r-1$. But this can be bounded via $\left\|\Delta D_{t}^{k} h\right\|_{L^{2}(\Omega)}$, given that $D_{t}^{k} h$ decays sufficiently fast at infinity (see Section 61 ).

In addition, apart from the coefficient in front of the highest order time derivative our energy does not depend in crucial way on $\kappa$ but uniformly (as $\kappa \rightarrow \infty$ ) control the corresponding norms of all but the highest order time derivative. This leads to that the a priori $L^{\infty}$ bounds also hold uniformly and the norms are bounded uniformly up to a fixed time. The convergence of solutions for the compressible Euler equations to the solution for the incompressible equations then follows from Arzela-Ascoli theorem.

Theorem 1.3. Let $u_{0}$ be a divergence free vector field such that its corresponding pressure $p_{0}$, defined by $\Delta p_{0}=-\left(\partial_{i} u_{0}^{k}\right)\left(\partial_{k} u_{0}^{i}\right)$ and $\left.p_{0}\right|_{\partial \mathcal{D}_{0}}=0$, satisfies the physical condition $-\left.\nabla_{N} p_{0}\right|_{\partial \mathcal{D}_{0}} \geq \epsilon>0$. Let $(u, p)$ be the solution of the incompressible free boundary Euler equations with data $u_{0}$, i.e.

$$
\rho_{0} D_{t} u=-\partial p, \quad \operatorname{div} u=0,\left.\quad p\right|_{\partial \mathcal{D}_{0}}=0,\left.\quad u\right|_{t=0}=u_{0}
$$

with the constant density $\rho_{0}=1$. Furthermore, let $\left(v_{\kappa}, h_{\kappa}\right)$ be the solution for the compressible Euler equations (1.30), with the density function $\rho_{\kappa}: h \rightarrow \rho_{\kappa}(h)$, and the initial data $v_{0 \kappa}$ and $\left.h_{\kappa}\right|_{t=0}=h_{0 \kappa}$, satisfying the compatibility condition (1.6) up to order $r+1$, as well as the physical sign condition (1.7). Suppose that $\rho_{\kappa} \rightarrow \rho_{0}=1, v_{0 \kappa} \rightarrow u_{0}$ and $h_{0 \kappa} \rightarrow p_{0}$ as $\kappa \rightarrow \infty$, such that $E_{r, \kappa}^{*}(0)$ is bounded uniformly independent of $\kappa$, then

$$
\left(v_{\kappa}, h_{\kappa}\right) \rightarrow(u, p)
$$


Remark. It is essential to make sure that the data satisfies (1.6) in Theorem 1.3 A good example is that if one starts with $v_{0}=u_{0}$, where $\operatorname{div} u_{0}=0$ and $h_{0}=0$ (e.g., $\left.\rho_{0}=1\right)$, then it is easy to see, after taking divergence on both sides of first equation of (1.30), $\left.D_{t}^{2} h\right|_{t=0}=\left(\partial u_{0}\right) \cdot\left(\partial u_{0}\right)$, and this would in general contradicts that $D_{t}^{2} h=0$ on $\partial \mathcal{D}_{t}$. To prevent this from happening, we give data in terms of enthalpy $h$, and hence $\rho \sim 1+h / \kappa$. We are then able to construct initial data via solving a system of elliptic equations.

In Section 7 we prove that there exist initial data satisfying the conditions in Theorem 1.3 in weighted Sobolev spaces $H_{w}^{r+1}(\Omega)$ with $w(x)=\left(1+|x|^{2}\right)^{\mu}, \mu \geq 2$ when $\kappa$ is sufficiently large. In particular, we prove:

Theorem 1.4. Let $u_{0}$ and $p_{0}$ are the initial data for the incompressible Euler equations defined in Theorem 1.3. and we further assume $u_{0} \in H_{w}^{s}\left(\mathcal{D}_{0}\right)$ for $s \geq r+1$, where $r>\frac{n}{2}+\frac{3}{2}$, if $\mathcal{D}_{0}$ is unbounded, diffeomprphic to the half space. Let $\rho_{\kappa}(h) \sim \rho_{0}+h / \kappa$, then there exists initial data $v_{0 \kappa}$ and $h_{0 \kappa}$ satisfying the compatibility condition (1.6) up to order $r+1$, such that $v_{0 \kappa} \rightarrow u_{0}, h_{0 \kappa} \rightarrow p_{0}$ as $\kappa \rightarrow \infty$, and $E_{r, \kappa}^{*}(0)$ (and hence $\widetilde{E}_{r, \kappa}^{*}(0)$ ) is uniformly bounded for all $\kappa$.

Remark. Theorem 1.4 implies that we need the initial data to be in $H_{w}^{5}$ when $n=3$ and in $H_{w}^{4}$ when $n=2$.

In addition to this, we show that the physical sign condition (1.7) can be verified via the maximum principle when the liquid is assumed to be irrotational. Finally, Section 8 is devoted to prove the weighted energy estimates for the compressible Euler equations, as an analogue to Theorem 1.1.

Theorem 1.5. Let $E_{w, r}$ be defined as (1.12) in $L_{w}^{2}\left(\mathcal{D}_{t}\right)$ with weight $w(x)=\left(1+|x|^{2}\right)^{\mu}, \mu \geq 2$. Then for each fixed $r \geq 1$, we have

$$
\left|\frac{d E_{w, r}(t)}{d t}\right| \leq C_{r}\left(K, \frac{1}{\epsilon}, M, c_{0}, E_{w, r-1}^{*}\right) E_{w, r}^{*}(t),
$$

provided the (1.5) and a priori assumptions (1.24)-(1.29).

Theorem 1.5 suggests that one should also be able to prove the (local) existence for localized solutions, given localized initial data constructed in Theorem 1.4 which serves as a good preparation for proving dispersive estimates and hence long time existence for a compressible water wave.

\section{The Lagrangian coordinates}

Let us first introduce Lagrangian coordinate, under which the boundary becomes fixed. Let $\Omega=\left\{x \in \mathbb{R}^{n}\right.$ : $\left.x_{n} \leq 0\right\}$ be the half space in $\mathbb{R}^{n}$, and let $f_{0}: \Omega \rightarrow \mathcal{D}_{0}$ to be a diffeomorphism. The Lagrangian coordinate $(t, y)$ where $x=x(t, y)=f_{t}(y)$ are given by solving

$$
\frac{d x}{d t}=v(t, x(t, y)), \quad x(0, y)=f_{0}(y), \quad y \in \Omega
$$

The boundary becomes fixed in the new coordinate, and we introduce the notation

$$
D_{t}=\left.\frac{\partial}{\partial t}\right|_{y=\text { constant }}=\left.\frac{\partial}{\partial t}\right|_{x=\text { constant }}+v^{k} \frac{\partial}{\partial x^{k}},
$$

to be the material derivative and

$$
\partial_{i}=\frac{\partial}{\partial x^{i}}=\frac{\partial y^{a}}{\partial x^{i}} \frac{\partial}{\partial y^{a}}
$$

Due to (2.2), we shall also call $D_{t}$ as the time derivative as well by slightly abuse of terminology.

Sometimes it is convenient to work in the Eulerian coordinate $(t, x)$, and sometimes it is easier to work in the Lagrangian coordinate $(t, y)$. In the Lagrangian coordinate the partial derivative $\partial_{t}=D_{t}$ has more direct significance than it in the Eulerian frame. However, this is not true for spatial derivatives $\partial_{i}$. The notion of space derivative that plays a more significant role in the Lagrangian coordinate is that the covariant differentiation with respect to the metric $g_{a b}(t, y)$. We shall not involve covariant derivatives in our energy; instead, we use the regular Eulerian spatial derivatives. We will work mostly in the Lagrangian coordinate 
in this paper. However, our statements are coordinate independent.

The Euclidean metric $\delta_{i j}$ in $\mathcal{D}_{t}$ induces a metric

$$
g_{a b}(t, y)=\delta_{i j} \frac{\partial x^{i}}{\partial y^{a}} \frac{\partial x^{j}}{\partial y^{b}}
$$

in $\Omega$ for each fixed $t$. We will denote covariant differentiation in the $y_{a}$-coordinate by $\nabla_{a}, a=1, \cdots, n$, and the differentiation in the $x_{i}$-coordinate by $\partial_{i}, i=1, \cdots, n$. Here, we use the convention that differentiation with respect to Eulerian coordinates is denoted by letters $i, j, k, l$ and with respect to Lagrangian coordinate is denoted by $a, b, c, d$.

The regularity of the boundary is measured by the regularity of the normal, let $N^{a}$ to be the unit normal to $\partial \Omega$,

$$
g_{a b} N^{a} N^{b}=1,
$$

and let $N_{a}=g_{a b} N^{b}$ denote the unit co-normal, $g^{a b} N_{a} N_{b}=1$. The induced metric $\gamma$ on the tangent space to the boundary $T(\partial \Omega)$ extended to be 0 on the orthogonal complement in $T(\Omega)$ is given by

$$
\gamma_{a b}=g_{a b}-N_{a} N_{b}, \quad \gamma^{a b}=g^{a c} g^{b d} \gamma_{c d}=g^{a b}-N^{a} N^{b} .
$$

The orthogonal projection of an $(0, r)$ tensor $S$ to the boundary is given by

$$
(\Pi S)_{a_{1}, \cdots, a_{r}}=\gamma_{a_{1}}^{b_{1}} \cdots \gamma_{a_{r}}^{b_{r}} S_{b_{1}, \cdots, b_{r}},
$$

where $\gamma_{a}^{b}=g^{b c} \gamma_{a c}=\delta_{a}^{b}-N_{a} N^{b}$. In particular, the covariant differentiation on the boundary $\bar{\nabla}$ is given by

$$
\bar{\nabla} S=\Pi \nabla S
$$

Note that $\bar{\nabla}$ is invariantly defined since the projection and $\nabla$ are. The second fundamental form of the boundary $\theta$ is given by $\theta_{a b}=(\bar{\nabla} N)_{a b}$, and the mean curvature of the boundary $\sigma=\operatorname{tr} \theta=g^{a b} \theta_{a b}$.

It is now important to compute time derivative of the metric $D_{t} g$, as well as the normal $D_{t} N$, as well as the time derivative of corresponding measures.

Theorem 2.1. Let $x=f_{t}(y)=x(t, y)$ be the change of variable given by (2.1) and $g$ be the metric given by (2.3), and $\gamma_{a b}=g_{a b}-N_{a} N_{b}$, where $N_{a}=g_{a b} N^{b}$ is the co-normal to $\partial \Omega$, set

$$
\begin{array}{r}
v_{a}(t, y)=v_{i}(t, x) \frac{\partial x^{i}}{\partial y^{a}}, \\
u^{a}=g^{a b} u_{b},
\end{array}
$$

$d \mu_{g}, \quad$ volume element with respect to the metric $g$,

$d \mu_{\gamma}, \quad$ surface element with respect to the metric $\gamma$.

Then

$$
\begin{array}{r}
D_{t} g_{a b}=\nabla_{a} v_{b}+\nabla_{b} v_{a}, \\
D_{t} g^{a b}=-g^{a c} g^{b d} D_{t} g_{c d}, \\
D_{t} N_{a}=-\frac{1}{2} N_{a}\left(D_{t} g^{c d}\right) N_{c} N_{d}, \\
D_{t} d \mu_{g}=\operatorname{div} v d \mu_{g}, \\
D_{t} d \mu_{\gamma}=(\sigma v \cdot N) d \mu_{\gamma} .
\end{array}
$$

Proof. The detail proof can be found in [20]. 


\section{Basic estimates on the domain with free boundary}

Most of the results in this section will be stated in a coordinate-independent fashion. Throughout this section, $\nabla$ will refer to covariant derivative with respect to the metric $g_{i j}$ in $\Omega$, and $\bar{\nabla}$ will refer to covariant differentiation on $\partial \Omega$ with respect to the induced metric $\gamma_{i j}=g_{i j}-N_{i} N_{j}$. Hence, in this section, $\Omega$ will be used to denote a general domain with smooth boundary. In addition, we shall assume the normal $N$ to $\partial \Omega$ is extended to a vector field in the interior of $\Omega$ satisfying $g_{i j} N^{i} N^{j} \leq 1$ by the same way introduced in lemma 2.1 .

\subsection{Elliptic estimates}

Definition 3.1. Let $u: \Omega \subset \mathbb{R}^{n} \rightarrow \mathbb{R}^{n}$ be a smooth vector field, and $\beta_{k}=\beta_{I k}=\nabla_{I}^{r} u_{k}$ be the $(0, r)$-tensor defined based on $u_{k}$, where $\nabla_{I}^{r}=\nabla_{i_{1}} \cdots \nabla_{i_{r}}$ and $I=\left(i_{1}, \cdots, i_{r}\right)$ is the set of indices. Let $\operatorname{div} \beta_{k}=\nabla_{i} \beta^{i}=$ $\nabla^{r} \operatorname{div} u$ and $\operatorname{curl} \beta=\nabla_{i} \beta_{j}-\nabla_{j} \beta_{i}=\nabla^{r} \operatorname{curl} u_{i j}$.

Definition 3.2. (Norms) If $|I|=|J|=r$, let $g^{I J}=g^{i_{1} j_{1}} \cdots g^{i_{r} j_{r}}$ and $\gamma^{I J}=\gamma^{i_{1} j_{1}} \cdots \gamma^{i_{r} j_{r}}$. If $\alpha, \beta$ are $(0, r)$ tensors, let $\langle\alpha, \beta\rangle=g^{I J} \alpha_{I} \beta_{J}$ and $|\alpha|^{2}=\langle\alpha, \alpha\rangle$. If $(\Pi \beta)_{I}=\gamma_{I}^{J} \beta_{J}$ is the projection, then $\langle\Pi \alpha, \Pi \beta\rangle=$ $\gamma^{I J} \alpha_{I} \beta_{J}$.Let

$$
\begin{array}{r}
\|\beta\|_{L^{2}(\Omega)}=\left(\int_{\Omega}|\beta|^{2} d \mu_{g}\right)^{\frac{1}{2}}, \\
\|\beta\|_{L^{2}(\partial \Omega)}=\left(\int_{\partial \Omega}|\beta|^{2} d \mu_{\gamma}\right)^{\frac{1}{2}}, \\
\|\Pi \beta\|_{L^{2}(\partial \Omega)}=\left(\int_{\partial \Omega}|\Pi \beta|^{2} d \mu_{\gamma}\right)^{\frac{1}{2}} .
\end{array}
$$

We now state the following Hodge-type decomposition theorem, which serves as a main ingredient for proving the elliptic estimates.

Theorem 3.1. (Hodge-decomposition) Let $\beta$ be defined in Definition 3.1 If $|\theta|+\left|\frac{1}{l_{0}}\right| \leq K$, where $\theta$ is the second fundamental form and $l_{0}$ is the injective radius defined in (1.10), then

$$
\begin{array}{r}
|\nabla \beta|^{2} \lesssim g^{i j} \gamma^{k l} \gamma^{I J} \nabla_{k} \beta_{I i} \nabla_{l} \beta_{J j}+|\operatorname{div} \beta|^{2}+|\operatorname{curl} \beta|^{2}, \\
\int_{\Omega}|\nabla \beta|^{2} d \mu_{g} \lesssim \int_{\Omega}\left(N^{i} N^{j} g^{k l} \gamma^{I J} \nabla_{k} \beta_{I i} \nabla_{l} \beta_{J j}+|\operatorname{div} \beta|^{2}+|\operatorname{curl} \beta|^{2}+K^{2}|\beta|^{2}\right) d \mu_{g}
\end{array}
$$

Proof. See [5]; we also refer Section 8 for the weighted version.

Proposition 3.2. (Elliptic estimates) Let $q: \Omega \rightarrow \mathbb{R}$ be a smooth function. Suppose that $|\theta|+\left|\frac{1}{l_{0}}\right| \leq K$, then we have, for any $r \geq 2$ and $\delta>0$,

$$
\begin{array}{r}
\left\|\nabla^{r} q\right\|_{L^{2}(\partial \Omega)} \lesssim K \sum_{s \leq r}\left\|\Pi \nabla^{s} q\right\|_{L^{2}(\partial \Omega)}+\sum_{s \leq r-1}\left\|\nabla^{s} \Delta q\right\|_{L^{2}(\Omega)}+\|\nabla q\|_{L^{2}(\Omega)}, \\
\left\|\nabla^{r} q\right\|_{L^{2}(\Omega)} \lesssim K \delta \sum_{s \leq r}\left\|\Pi \nabla^{s} q\right\|_{L^{2}(\partial \Omega)}+\delta^{-1} \sum_{s \leq r-2}\left\|\nabla^{s} \Delta q\right\|_{L^{2}(\Omega)}+\delta^{-1}\|\nabla q\|_{L^{2}(\Omega)} .
\end{array}
$$

Proof. See [5; we also refer Section 8 for the weighted version.

Remark. We recall that if $\operatorname{vol} \Omega<\infty$, we have $\|\nabla q\|_{L^{2}(\Omega)} \leq C(\operatorname{vol} \Omega)\|\Delta q\|_{L^{2}(\Omega)}$.

\subsection{Estimate for the projection of a tensor to the tangent space of the boundary}

The use of the projection of the tensor $\Pi \nabla^{s} D_{t}^{k} h$ in the boundary part of energy (1.12) is essential to compensate the potential loss of regularity. A simple observation that will help us is that if $q=0$ on $\partial \Omega$, then $\Pi \nabla^{2} q$ contains only first-order derivative of $q$ and all components of the second fundamental form. To be more precise, we have

$$
\Pi \nabla^{2} q=\bar{\nabla}^{2} q+\theta \nabla_{N} q
$$


where the tangential component $\bar{\nabla}^{2} q=0$ on the boundary. Furthermore, in $L^{2}$ norms, (3.5) yields,

$$
\left\|\Pi \nabla^{2} q\right\|_{L^{2}(\partial \Omega)} \leq|\theta|_{L^{\infty}(\partial \Omega)}|| \nabla_{N} q \|_{L^{2}(\partial \Omega)} .
$$

To prove (3.5), we first recall the components of the projection operator $\gamma_{i}^{j}=\delta_{i}^{j}-N_{i} N^{j}$, hence

$$
\gamma_{j}^{k} \nabla_{i} \gamma_{k}^{l}=-\gamma_{j}^{k} \nabla_{i}\left(N_{k} N^{l}\right)=-\gamma_{j}^{k} \theta_{i k} N^{l}-\gamma_{j}^{k} N_{k} \theta_{i}^{l}=-\theta_{i j} N^{l},
$$

and so

$$
\begin{aligned}
\bar{\nabla}_{i} \bar{\nabla}_{j} q & =\gamma_{i}^{i^{\prime}} \gamma_{j}^{j^{\prime}} \nabla_{i^{\prime}} \gamma_{j^{\prime}}^{j^{\prime \prime}} \nabla_{j^{\prime \prime}} q \\
& =\gamma_{i}^{i^{\prime}} \gamma_{j}^{j^{\prime}} \gamma_{j^{\prime}}^{j^{\prime \prime}} \nabla_{i^{\prime}} \nabla_{j^{\prime \prime}} q+\gamma_{i}^{i^{\prime}} \gamma_{j}^{j^{\prime}}\left(\nabla_{i^{\prime}} \gamma_{j^{\prime}}^{j^{\prime \prime}}\right) \nabla_{j^{\prime \prime}} q \\
& =\gamma_{i}^{i^{\prime}} \gamma_{j}^{j^{\prime}} \nabla_{i^{\prime}} \nabla_{j^{\prime}} q-\theta_{i j} \nabla_{N} q .
\end{aligned}
$$

In general, the higher order projection formula is of the form

$$
\Pi \nabla^{r} q=\left(\bar{\nabla}^{r-2} \theta\right) \nabla_{N} q+O\left(\nabla^{r-1} q\right)+O\left(\bar{\nabla}^{r-3} \theta\right) .
$$

which suggests the following generalization of (3.6), its detailed proof can be found in [5].

Proposition 3.3. (Tensor estimate) Suppose that $|\theta|+\left|\frac{1}{l_{0}}\right| \leq K$, and for $q=0$ on $\partial \Omega$, then for $m=0,1$

$$
\begin{aligned}
& \left\|\Pi \nabla^{r} q\right\|_{L^{2}(\partial \Omega) \lesssim K}\left\|\left(\bar{\nabla}^{r-2} \theta\right) \nabla_{N} q\right\|_{L^{2}(\partial \Omega)}+\sum_{l=1}^{r-1}\left\|\nabla^{r-l} q\right\|_{L^{2}(\partial \Omega)} \\
& +\left(\|\theta\|_{L^{\infty}(\partial \Omega)}+\sum_{0 \leq l \leq r-2-m}\left\|\bar{\nabla}^{l} \theta\right\|_{L^{2}(\partial \Omega)}\right)\left(\sum_{0 \leq l \leq r-2+m}\left\|\nabla^{l} q\right\|_{L^{2}(\partial \Omega)}\right),
\end{aligned}
$$

where the second line drops for $0 \leq r \leq 4$.

\subsection{Estimate for the second fundamental form}

The estimate of the second fundamental form is a direct consequence of (3.7) with $q=h$ together with the physical sign condition,e.g., $\left|\nabla_{N} h\right| \geq \epsilon>0$.

Proposition 3.4. ( $\theta$ estimate) Suppose that $|\theta|+\left|\frac{1}{l_{0}}\right| \leq K$, and the physical sign condition $\left|\nabla_{N} h\right| \geq \epsilon>0$ holds, then

$$
\begin{aligned}
\left\|\bar{\nabla}^{r-2} \theta\right\|_{L^{2}(\partial \Omega) \lesssim} \lesssim_{K, \frac{1}{\epsilon}}\left\|\Pi \nabla^{r} h\right\|_{L^{2}(\partial \Omega)}+\sum_{s=1}^{r-1}\left\|\nabla^{r-s} h\right\|_{L^{2}(\partial \Omega)} & \\
& +\left(\|\theta\|_{L^{\infty}(\partial \Omega)}+\sum_{s \leq r-3}\left\|\bar{\nabla}^{s} \theta\right\|_{L^{2}(\partial \Omega)}\right) \sum_{s \leq r-1}\left\|\nabla^{s} h\right\|_{L^{2}(\partial \Omega)},
\end{aligned}
$$

where the second line drops for $0 \leq r \leq 4$.

\section{The wave equation}

In this section we study the wave equation satisfied by $h$, obtained by commutating $D_{t}$ with the first equation of (1.3)

$$
D_{t}^{2} e(h)-\Delta h=\left(\partial_{i} v^{j}\right)\left(\partial_{j} v^{i}\right), \quad \text { in } \quad[0, T] \times \Omega
$$

with initial and boundary conditions

$$
\left.h\right|_{t=0}=h_{0},\left.\quad D_{t} h\right|_{t=0}=h_{1},
$$


and

$$
\left.h\right|_{\partial \Omega}=0 .
$$

Here, $\Delta=\delta^{i j} \partial_{i} \partial_{j}=\frac{1}{\sqrt{|\operatorname{det} g|}} \partial_{a}\left(\sqrt{|\operatorname{det} g|} g^{a b} \partial_{b}\right)$. In order to express the higher order tensor products in a more appealing way, we adopt the following notation introduced in [5].

Definition 4.1. (Symmetric dot product) Let $\left[D_{t}, \partial\right]=-(\partial v) \sim \partial$, where the symmetric dot product $(\partial v) \sim \partial$ is define component-wisely by $\left((\partial v)^{\sim} \cdot \partial\right)_{i}=\partial_{i} v^{k} \partial_{k}$. In general, we have

$$
\left[D_{t}, \partial^{r}\right]=\sum_{s=0}^{r-1} \partial^{s}\left[D_{t}, \partial\right] \partial^{r-s-1}=\sum_{s=0}^{r-1}-\left(\begin{array}{c}
r \\
s+1
\end{array}\right)\left(\partial^{1+s} v\right)^{2} \cdot \partial^{r-s}
$$

where

$$
\left(\left(\partial^{1+s} v\right) \cdot \partial^{r-s}\right)_{i_{1}, \cdots, i_{r}}=\frac{1}{r !} \sum_{\sigma \in S_{r}}\left(\partial_{i_{\sigma_{1}} \cdots i_{\sigma_{1+s}}}^{1+s} v^{k}\right)\left(\partial_{k, i_{\sigma_{s+2} \cdots \sigma_{r}}}^{s}\right)
$$

where $S_{r}$ is the $r$-symmetric group.

In addition, the commutators between $D_{t}^{k}$ for $k \geq 2$ and spatial derivatives can be expressed as

$$
\left[\partial, D_{t}^{k}\right]=\sum_{l_{1}+l_{2}=k-1} c_{l_{1}, l_{2}}\left(\partial D_{t}^{l_{1}} v\right) \tilde{*}\left(\partial D_{t}^{l_{2}}\right)+\sum_{l_{1}+\cdots+l_{n}=k-n+1, n \geq 3} d_{l_{1}, \cdots, l_{n}}\left(\partial D_{t}^{l_{1}} v\right) \cdots\left(\partial D_{t}^{l_{n-1}} v\right)\left(\partial D_{t}^{l_{n}}\right),
$$

and

$$
\begin{aligned}
{\left[\Delta, D_{t}^{r-1}\right]=\sum_{l_{1}+l_{2}=r-2} } & c_{l_{1}, l_{2}}\left(\Delta D_{t}^{l_{1}} v\right) \cdot\left(\partial D_{t}^{l_{2}}\right)+\sum_{l_{1}+l_{2}=r-2} c_{l_{1}, l_{2}}\left(\partial D_{t}^{l_{1}} v\right) \cdot\left(\partial^{2} D_{t}^{l_{2}}\right) \\
& +\sum_{l_{1}+\cdots+l_{n}=r-n, n \geq 3} d_{l_{1}, \cdots, l_{n}}\left(\partial D_{t}^{l_{3}} v\right) \cdots\left(\partial D_{t}^{l_{n}} v\right) \cdot\left(\Delta D_{t}^{l_{1}} v\right) \cdot\left(\partial D_{t}^{l_{2}}\right) \\
& +\sum_{l_{1}+\cdots+l_{n}=r-n, n \geq 3} e_{l_{1}, \cdots, l_{n}}\left(\partial D_{t}^{l_{3}} v\right) \cdots\left(\partial D_{t}^{l_{n}} v\right) \cdot\left(\partial^{2} D_{t}^{l_{1}} v\right) \cdot\left(\partial D_{t}^{l_{2}}\right) \\
& \quad+\sum_{l_{1}+\cdots+l_{n}=r-n, n \geq 3} f_{l_{1}, \cdots, l_{n}}\left(\partial D_{t}^{l_{3}} v\right) \cdots\left(\partial D_{t}^{l_{n}} v\right) \cdot\left(\partial D_{t}^{l_{1}} v\right) \cdot\left(\partial^{2} D_{t}^{l_{2}}\right),
\end{aligned}
$$

where the regular dot product is defined to be the trace of the symmetric dot.

\subsection{The Energies $W_{r}(t)$}

By commutating $D_{t}^{r-1}$ on both sides of (4.1), we obtain the higher order wave equation

$$
e^{\prime}(h) D_{t}^{r+1} h-\Delta D_{t}^{r-1} h=f_{r}+g_{r},
$$

where

$$
f_{r}=D_{t}^{r-1}(\partial v \cdot \partial v)+\left[D_{t}^{r-1}, \Delta\right] h
$$

and $g_{r}$ is sum of terms of the form

$$
e^{(m)}(h)\left(D_{t}^{i_{1}} h\right) \cdots\left(D_{t}^{i_{m}} h\right), \quad 2 \leq m \leq r, \quad i_{1}+\cdots+i_{m}=r+1, \quad 1 \leq i_{1} \leq \cdots \leq i_{m} \leq r .
$$

Now, let us define the energy

$$
W_{r}(t)=\frac{1}{2}\left\|\sqrt{e^{\prime}(h)} D_{t}^{r} h\right\|_{L^{2}(\Omega)}+\frac{1}{2}\left\|\nabla D_{t}^{r-1} h\right\|_{L^{2}(\Omega)}
$$

and by the standard energy estimates for the wave equations together with (4.5), we have

Theorem 4.1. Let $W_{r}$ be defined as in (4.10), we have

$$
\frac{d W_{r}}{d t} \lesssim W_{r}+\left\|f_{r}\right\|_{L^{2}(\Omega)}+\left\|g_{r}\right\|_{L^{2}(\Omega)} .
$$

Proof. This follows from standard energy estimates for the wave equation. We refer [20] Section 4 for the detail. 


\subsection{Estimates for $\left\|f_{r}\right\|_{L^{2}(\Omega)}$}

By adopting our notations used in (4.5)-(4.6), we are able to express $f_{r}$ as

$$
\begin{aligned}
f_{r}= & \sum_{l_{1}+l_{2}=r-1} c_{l_{1}, l_{2}}\left(\nabla D_{t}^{l_{1}} v\right) \cdot\left(\nabla D_{t}^{l_{2}} v\right)+\sum_{l_{1}+l_{2}=r-2} d_{l_{1}, l_{2}}\left(\Delta D_{t}^{l_{1}} v\right) \cdot\left(\nabla D_{t}^{l_{2}} h\right) \\
& +\sum_{l_{1}+l_{2}=r-2} e_{l_{1}, l_{2}}\left(\nabla D_{t}^{l_{1}} v\right) \cdot\left(\nabla^{2} D_{t}^{l_{2}} h\right)+\text { error terms }
\end{aligned}
$$

where the "error terms" refer to the terms generated by the commutators, which are of the form

$$
\begin{aligned}
e_{r}=\sum_{l_{1}+\cdots+l_{n}=r+1-n, n \geq 3} & g_{l_{1}, \cdots, l_{n}}\left(\partial D_{t}^{l_{3}} v\right) \cdots\left(\partial D_{t}^{l_{n}} v\right) \cdot\left(\partial D_{t}^{l_{1}} v\right) \cdot\left(\partial D_{t}^{l_{2}} v\right) \\
+\sum_{l_{1}+\cdots+l_{n}=r-n, n \geq 3} \sum_{l_{1}, \cdots, l_{n}}\left(\partial D_{t}^{l_{3}} v\right) \cdots\left(\partial D_{t}^{l_{n}} v\right) \cdot\left(\partial^{2} D_{t}^{l_{1}} v\right) \cdot\left(\partial D_{t}^{l_{2}} h\right) & \sum_{l_{1}+\cdots+l_{n}=r-n, n \geq 3} f_{l_{1}, \cdots, l_{n}}\left(\partial D_{t}^{l_{3}} v\right) \cdots\left(\partial D_{t}^{l_{n}} v\right) \cdot\left(\partial D_{t}^{l_{1}} v\right) \cdot\left(\partial^{2} D_{t}^{l_{2}} h\right) .
\end{aligned}
$$

We need to estimate $\left\|f_{r}\right\|_{L^{2}(\Omega)}$ and $\left\|g_{r}\right\|_{L^{2}(\Omega)}$ for $r \geq 1$. Since our estimates include mixed space-time derivatives, we would like to use the following more appealing notations.

Definition 4.2. (Mixed Sobolev norms) let $u(t, \cdot): \mathbb{R}^{n} \rightarrow \mathbb{R}$ be a smooth function. We define

$$
\begin{gathered}
\|u\|_{r, 0}=\sum_{s+k=r, k<r}\left\|\nabla^{s} D_{t}^{k} u\right\|_{L^{2}(\Omega)}, \\
\|u\|_{r}=\|u\|_{r, 0}+\left\|\sqrt{e^{\prime}(h)} D_{t}^{r} h\right\|_{L^{2}(\Omega)} .
\end{gathered}
$$

We have to make sure that the $r$-th order Sobolev norms in our estimates for $\left\|f_{r}\right\|_{L^{2}(\Omega)}, r \geq 3$ do not include $\left\|\nabla^{r} h\right\|_{L^{2}(\Omega)}$ and $\left\|\nabla^{r} v\right\|_{L^{2}(\Omega)}$. This is because that we need to control $\left\|f_{r+1}\right\|_{L^{2}(\Omega)}, r \geq 2$ by $\sqrt{E_{r}^{*}}$ in Section 5.5. but $\left\|\nabla^{r+1} h\right\|_{L^{2}(\Omega)}$ and $\left\|\nabla^{r+1} v\right\|_{L^{2}(\Omega)}$ can only be controlled by $\sqrt{E_{r+1}^{*}}$.

\subsubsection{When $\mathrm{r}=1,2,3$}

Since $f_{1}=(\nabla v) \cdot(\nabla v)$, we have

$$
\left\|f_{1}\right\|_{L^{2}(\Omega)} \lesssim M\|\nabla v\|_{L^{2}(\Omega)} .
$$

The bounds for $\left\|f_{2}\right\|_{L^{2}(\Omega)}$ and $\left\|f_{3}\right\|_{L^{2}(\Omega)}$ are the same as what we did in our previous work [20], and so we shall only record results here.

$$
\begin{gathered}
\left\|f_{2}\right\|_{L^{2}(\Omega) \lesssim M}\left\|\nabla^{2} v\right\|_{L^{2}(\Omega)}+\left\|\nabla^{2} h\right\|_{L^{2}(\Omega)}+\|\nabla v\|_{L^{2}(\Omega)} . \\
\left\|f_{3}\right\|_{L^{2}(\Omega)} \lesssim_{M}\left\|\nabla^{2} D_{t} h\right\|_{L^{2}(\Omega)}+\left\|e^{\prime}(h) \nabla D_{t}^{2} h\right\|_{L^{2}(\Omega)}+\|h\|_{2,0}+\sum_{j=1,2}\left\|\nabla^{j} v\right\|_{L^{2}(\Omega)} .
\end{gathered}
$$

\subsubsection{When $\mathrm{r}=4$}

The bounds for the first and the third term of $f_{4}$ is the same as in [20].

$$
\begin{aligned}
& \sum_{l_{1}+l_{2}=3}\left\|c_{l_{1}, l_{2}}\left(\nabla D_{t}^{l_{1}} v\right)\left(\nabla D_{t}^{l_{2}} v\right)\right\|_{L^{2}(\Omega)}+\sum_{l_{1}+l_{2}=2}\left\|e_{l_{1}, l_{2}}\left(\nabla D_{t}^{l_{1}} v\right)\left(\nabla^{2} D_{t}^{l_{2}} h\right)\right\|_{L^{2}(\Omega)} \\
& \lesssim_{M}\left\|\nabla^{2} D_{t}^{2} h\right\|_{L^{2}(\Omega)}+\sum_{j=2,3}\|h\|_{j, 0}+\left\|\nabla^{2} v\right\|_{L^{2}(\Omega)}+\left\|\nabla D_{t}^{2} v\right\|_{L^{2}(\Omega)} .
\end{aligned}
$$

But we cannot use interpolation to bound $\left\|\Delta v \cdot \nabla D_{t}^{2} h\right\|_{L^{2}(\Omega)}$ involved in the second term of $f_{4}$, as $\left|D_{t}^{2} h\right|$ is no longer part of the a priori assumptions. But since

$$
\Delta v=\nabla \operatorname{div} v+\nabla \cdot \operatorname{curl} v
$$


and since $\left|e^{\prime \prime}(h)\right| \leq c_{0}\left|e^{\prime}(h)\right|$,

$$
|\nabla \operatorname{div} v| \lesssim\left|e^{\prime}(h)(\nabla h) D_{t} h\right|+\left|e^{\prime}(h) \nabla D_{t} h\right|
$$

is bounded by a priori assumptions (1.28) and (1.29). On the other hand 1 , since $|\nabla \cdot \operatorname{curl} v| \leq M$ as well, we conclude

$$
\left\|\Delta v \cdot \nabla D_{t}^{2} h\right\|_{L^{2}(\Omega)} \lesssim_{M}\left\|\nabla D_{t}^{2} h\right\|_{L^{2}(\Omega)},
$$

and so

$$
\sum_{l_{1}+l_{2}=2}\left\|\left(\Delta D_{t}^{l_{1}} v\right) \cdot\left(\nabla D_{t}^{l_{2}} h\right)\right\|_{L^{2}(\Omega)} \lesssim M\left\|\nabla D_{t}^{2} h\right\|_{L^{2}(\Omega)}+\left\|\nabla^{3} D_{t} h\right\|_{L^{2}(\Omega)}+\sum_{j=2,3}\left(\left\|\nabla^{j} v\right\|_{L^{2}(\Omega)}+\left\|\nabla^{j} h\right\|_{L^{2}(\Omega)}\right) .
$$

Most of the terms in $e_{4}$ can be bounded by corresponding terms in $f_{r}$, for $r \leq 4$, and similar terms in $e_{3}$ times a priori assumptions, apart from terms of the form $\nabla v \cdot \nabla^{2} D_{t} v \cdot \nabla h$, whose $L^{2}$ norm can be bounded by $\left\|\nabla^{3} h\right\|_{L^{2}(\Omega)}$.

Therefore, we sum up and get

$$
\left\|f_{4}\right\|_{L^{2}(\Omega)} \lesssim M\left\|\nabla^{3} D_{t} h\right\|_{L^{2}(\Omega)}+\left\|\nabla^{2} D_{t}^{2} h\right\|_{L^{2}(\Omega)}+\sum_{j=2,3}\left(\|h\|_{j, 0}+\left\|\nabla^{j} v\right\|_{L^{2}(\Omega)}\right) .
$$

\subsubsection{When $r=5$ and $n \leq 4$}

The bounds for the first and the third terms of $f_{5}$ remains unchanged as in [20].

$$
\begin{aligned}
\sum_{l_{1}+l_{2}=4}\left\|\left(\nabla D_{t}^{l_{1}} v\right)\left(\nabla D_{t}^{l_{2}} v\right)\right\|_{L^{2}(\Omega)}+\sum_{l_{1}+l_{2}=3}\left\|\left(\nabla D_{t}^{l_{1}} v\right)\left(\nabla^{2} D_{t}^{l_{2}} h\right)\right\|_{L^{2}(\Omega)} \\
\quad \lesssim_{K, M}\left\|\nabla^{2} D_{t}^{3} h\right\|_{L^{2}(\Omega)}+\sum_{1 \leq i \leq 4}\|v\|_{i, 0}+\sum_{2 \leq i \leq 4}\|h\|_{i, 0} .
\end{aligned}
$$

As for the term $\sum_{l_{1}+l_{2}=3} d_{l_{1}, l_{2}}\left\|\left(\Delta D_{t}^{l_{1}} v\right)\left(\nabla D_{t}^{l_{2}} h\right)\right\|_{L^{2}(\Omega)}$, we need the Sobolev lemma (A.4) to bound $\| \Delta v$. $\nabla D_{t}^{3} h \|_{L^{2}(\Omega)}$ and $\left\|\Delta D_{t} v \cdot \nabla D_{t}^{2} h\right\|_{L^{2}(\Omega)}$ as in [20, i.e.,

$$
\begin{gathered}
\left\|\Delta v \cdot \nabla D_{t}^{3} h\right\|_{L^{2}(\Omega)} \lesssim_{K}\left(\sum_{j=2,3}\left\|\nabla^{j} v\right\|_{L^{2}(\Omega)}\right)\left(\sum_{j=1,2}\left\|\nabla^{j} D_{t}^{3} h\right\|_{L^{2}(\Omega)}\right), \\
\left\|\Delta D_{t} v \cdot \nabla D_{t}^{2} h\right\|_{L^{2}(\Omega)} \lesssim_{K}\left(\sum_{j=3,4}\left\|\nabla^{j} h\right\|_{L^{2}(\Omega)}\right)\left(\sum_{j=1,2}\left\|\nabla^{j} D_{t}^{2} h\right\|_{L^{2}(\Omega)}\right), \\
\left\|\Delta D_{t}^{2} v \cdot \nabla D_{t} h\right\|_{L^{2}(\Omega)} \lesssim M\left\|\nabla^{2} D_{t}^{2} v\right\|_{L^{2}(\Omega)} \lesssim_{M}\left\|\nabla^{3} D_{t} h\right\|_{L^{2}(\Omega)}+\sum_{j \leq 3}\left(\left\|\nabla^{j} v\right\|_{L^{2}(\Omega)}+\left\|\nabla^{j} h\right\|_{L^{2}(\Omega)}\right),
\end{gathered}
$$

and

$$
\begin{aligned}
& \left\|\Delta D_{t}^{3} v \cdot \nabla h\right\|_{L^{2}(\Omega)} \lesssim_{M}\left\|\nabla \Delta D_{t}^{2} h\right\|_{L^{2}(\Omega)}+\left\|\Delta\left[D_{t}^{2}, \nabla\right] h\right\|_{L^{2}(\Omega)} \\
& \quad{ }_{K, M}\left\|\nabla^{3} D_{t}^{2} h\right\|_{L^{2}(\Omega)}+\sum_{j=2,3}\|v\|_{i, 0}+\sum_{j=3,4}\|h\|_{i, 0},
\end{aligned}
$$

respectively. Most of the terms in the error term $e_{5}$ are essentially bounded by corresponding terms in $f_{r}$, for $r \leq 5$, and similar terms in $e_{3}$ and $e_{4}$ times a priori assumptions, apart from the terms of the form $\nabla v \cdot \nabla^{2} D_{t}^{2} v \cdot \nabla h$, which is estimated by $\left\|\nabla^{2} D_{t}^{2} v\right\|_{L^{2}(\Omega)}$. Hence,

$$
\begin{aligned}
& \left\|f_{5}\right\|_{L^{2}(\Omega)} \lesssim K, M\left\|\nabla^{3} D_{t}^{2} h\right\|_{L^{2}(\Omega)}+\left(\sum_{j=2,3}\left\|\nabla^{j} v\right\|_{L^{2}(\Omega)}\right)\left(\sum_{j=1,2}\left\|\nabla^{j} D_{t}^{3} h\right\|_{L^{2}(\Omega)}\right) \\
& +\left(\sum_{j=3,4}\left\|\nabla^{j} h\right\|_{L^{2}(\Omega)}\right)\left(\sum_{j=1,2}\left\|\nabla^{j} D_{t}^{2} h\right\|_{L^{2}(\Omega)}\right)+\sum_{1 \leq i \leq 4}\|v\|_{i, 0}+\sum_{2 \leq i \leq 4}\|h\|_{i, 0} .
\end{aligned}
$$

\footnotetext{
${ }^{1}$ One could alternatively estimate $\left\|\Delta v \cdot \nabla D_{t}^{2} h\right\|_{L^{2}(\Omega)}$ by Sobolev lemma, e.g.,

$$
\left\|\Delta v \cdot \nabla D_{t}^{2} h\right\|_{L^{2}(\Omega)} \lesssim\left(\sum_{j=2,3}\left\|\nabla^{j} v\right\|_{L^{2}(\Omega)}\right)\left(\sum_{j=1,2}\left\|\nabla^{j} D_{t}^{2} h\right\|_{L^{2}(\Omega)}\right) .
$$
}

However, (1.23) then fails to be linear in $E_{r}^{*}$. 


\subsection{When $r \geq 6$}

The commutator (4.5) in fact implies that

$$
D_{t}^{k} v=-\partial D_{t}^{k-1} h+c_{\alpha^{\prime} \beta^{\prime} \gamma^{\prime}}\left(\partial^{\alpha_{1}^{\prime}} v\right) \cdots\left(\partial^{\alpha_{m}^{\prime}} v\right)\left(\partial^{\beta_{1}^{\prime}} D_{t}^{\gamma_{1}^{\prime}} h\right) \cdots\left(\partial^{\beta_{n}^{\prime}} D_{t}^{\gamma_{n}^{\prime}} h\right),
$$

where

$$
\begin{array}{r}
\alpha^{\prime}=\left(\alpha_{1}^{\prime}, \cdots, \alpha_{m}^{\prime}\right), \beta^{\prime}=\left(\beta_{1}^{\prime}, \cdots, \beta_{n}^{\prime}\right), \gamma^{\prime}=\left(\gamma_{1}^{\prime}, \cdots, \gamma_{n}^{\prime}\right), \\
\alpha_{1}^{\prime}+\cdots+\alpha_{m}^{\prime}+\left(\beta_{1}^{\prime}+\gamma_{1}^{\prime}\right)+\cdots+\left(\beta_{n}^{\prime}+\gamma_{n}^{\prime}\right)=k, \\
1 \leq \alpha_{i}^{\prime} \leq k-2, \quad \text { when } k \geq 3, \\
1 \leq \beta_{j}^{\prime} \leq k-2, \quad \text { when } \quad k \geq 4 .
\end{array}
$$

Because of this and (4.12), we can re-express $f_{r}, r \geq 6$ as

$$
f_{r}=c_{\alpha \beta \gamma}\left(\partial^{\alpha_{1}} v\right) \cdots\left(\partial^{\alpha_{m}} v\right)\left(\partial^{\beta_{1}} D_{t}^{\gamma_{1}} h\right) \cdots\left(\partial^{\beta_{n}} D_{t}^{\gamma_{n}} h\right),
$$

where

$$
\begin{array}{r}
\alpha=\left(\alpha_{1}, \cdots, \alpha_{m}\right), \beta=\left(\beta_{1}, \cdots, \beta_{n}\right), \gamma=\left(\gamma_{1}, \cdots, \gamma_{n}\right), \\
\alpha_{1}+\cdots+\alpha_{m}+\left(\beta_{1}+\gamma_{1}\right)+\cdots+\left(\beta_{n}+\gamma_{n}\right)=r+1, \\
1 \leq \alpha_{i} \leq r-2, \quad 1 \leq i \leq m, \\
1 \leq \beta_{j}+\gamma_{j} \leq r, \quad 1 \leq j \leq n .
\end{array}
$$

In addition to these, there exists at most one $i$ or $j$ such that $\alpha_{i}=r-2$ or $\beta_{j}+\gamma_{j} \geq r-2$, and further if $\beta_{j}+\gamma_{j} \geq r-1$, we must have $\gamma_{j} \geq 1$. Thus, $f_{r}$ never consists terms of the form $\left(\partial^{2} v\right)\left(\partial^{r-1} h\right)$ if $r \geq 6$.

Since $f_{r}$ is a sum of products of the form (4.20), we apply the following derivative counting method on each product to estimates $\left\|f_{r}\right\|_{L^{2}(\Omega)}$.

- If $\alpha_{i} \geq r-2$ for some $i$ or $\beta_{j}+\gamma_{j} \geq r-2$ for some $j$, then there are at most four terms involved in the product (4.20), among which at least one must satisfy a priori assumptions (1.24)-(1.29) if the product has more than two terms. Hence,

$$
\begin{aligned}
& \left\|\left(\nabla^{\alpha_{1}} v\right) \cdots\left(\nabla^{\alpha_{m}} v\right)\left(\nabla^{\beta_{1}} D_{t}^{\gamma_{1}} h\right) \cdots\left(\nabla^{\beta_{n}} D_{t}^{\gamma_{n}} h\right)\right\|_{L^{2}(\Omega)} \\
& \leq C_{r}\left(K, M, \sum_{k \leq r-2}\left\|\nabla^{k} v\right\|_{L^{2}(\Omega)}, \sum_{k \leq r-2}\|h\|_{k, 0}\right)\left(\sum_{k \leq r-1}\left\|\nabla^{k} v\right\|_{L^{2}(\Omega)}+\sum_{k \leq r-1}\|h\|_{k, 0}+\sum_{k \leq r-1}\left\|D_{t} h\right\|_{k, 0}\right) .
\end{aligned}
$$

Here we have used the Sobolev lemma

$$
\left\|u_{1} \cdots u_{N}\right\|_{L^{2}} \leq C(K)\left\|u_{1}\right\|_{H^{1}} \cdots\left\|u_{N}\right\|_{H^{1}}, \quad N=2,3
$$

Now, we assume $\alpha_{i} \leq r-3$ and $\beta_{j}+\gamma_{j} \leq r-3$ for all $i, j$.

- If $\alpha_{i}<r-3$ and $\beta_{j}+\gamma_{j}<r-3$ for all $i, j$, then

$$
\begin{aligned}
& \left\|\left(\nabla^{\alpha_{1}} v\right) \cdots\left(\nabla^{\alpha_{m}} v\right)\left(\nabla^{\beta_{1}} D_{t}^{\gamma_{1}} h\right) \cdots\left(\nabla^{\beta_{n}} D_{t}^{\gamma_{n}} h\right)\right\|_{L^{2}(\Omega)} \\
& \leq C_{r}\left(K, M, \sum_{k \leq r-2}\left\|\nabla^{k} v\right\|_{L^{2}(\Omega)}, \sum_{k \leq r-2}\|h\|_{k, 0}\right) .
\end{aligned}
$$

Here we have used the Sobolev lemma

$$
\left\|u_{1} \cdots u_{N}\right\|_{L^{2}} \leq C(K)\left\|u_{1}\right\|_{H^{2}} \cdots\left\|u_{N}\right\|_{H^{2}}, \quad N \geq 4 .
$$


- If $\alpha_{i}=r-3$ for some $i$ and/or $\beta_{j}+\gamma_{j}=r-3$ for some $j$, then there exists at most one $i^{\prime} \neq i$ or $j^{\prime} \neq j$ such that $\alpha_{i^{\prime}}=r-3$ or $\beta_{j^{\prime}}+\gamma_{j^{\prime}}=r-3$. In this case, the product consists at most 3 terms. Hence, (4.23) remains valid in this case by Sobolev lemma.

Therefore, one concludes that when $r \geq 6$,

$$
\begin{gathered}
\left\|f_{r}\right\|_{L^{2}(\Omega)} \leq C_{r}\left(K, M, \sum_{k \leq r-2}\left\|\nabla^{k} v\right\|_{L^{2}(\Omega)}, \sum_{k \leq r-2}\|h\|_{k, 0}\right) . \\
\left(\sum_{k \leq r-1}\left\|\nabla^{k} v\right\|_{L^{2}(\Omega)}+\sum_{k \leq r-1}\|h\|_{k, 0}+\sum_{k \leq r-1}\left\|D_{t} h\right\|_{k, 0}\right),
\end{gathered}
$$

where $C_{r}$ are continuous functions.

\subsection{Estimates for $\left\|g_{r}\right\|_{L^{2}(\Omega)}$}

We recall that $e(h)=\log \rho(h)$ which satisfies

1. $\left|e^{(k)}(h)\right| \leq c_{0}$.

2. $\left|e^{(k)}(h)\right| \leq c_{0} \sqrt{e^{\prime}(h)}$.

3. $\left|e^{(k)}(h)\right| \leq c_{0}\left|e^{\prime}(h)\right|^{k}$.

\subsection{When $r=1,2,3,4$}

For each $r, g_{r}$ is a sum of terms of the form

$$
e^{(m)}(h) D_{t}^{j_{1}} h \cdots D_{t}^{j_{m}} h, \quad j_{1}+\cdots+j_{m}=r+1, \quad 1 \leq j_{1} \leq \cdots \leq j_{m} \leq r,
$$

and $j_{i} \leq 2$ for $i \leq m-1$. Therefore, the a priori assumption (1.29) yields

$$
\left\|e^{(m)}(h) D_{t}^{j_{1}} h \cdots D_{t}^{j_{m}} h\right\|_{L^{2}(\Omega)} \lesssim\left\|\left(e^{\prime}(h) D_{t}^{j_{1}} h\right) \cdots\left(e^{\prime}(h) D_{t}^{j_{m}} h\right)\right\|_{L^{2}(\Omega)} \lesssim M, c_{0}\left\|e^{\prime}(h) D_{t}^{j_{m}} h\right\|_{L^{2}(\Omega)} .
$$

Hence we conclude

$$
\left\|g_{r}\right\|_{L^{2}(\Omega)} \lesssim M, c_{0} \sum_{j \leq r}\left\|e^{\prime}(h) D_{t}^{j} h\right\|_{L^{2}(\Omega)}, \quad r \leq 4
$$

\subsection{When $r=5$}

The only difference for estimating $g_{5}$ is that it contains a quadratic term $e^{\prime \prime}(h) D_{t}^{3} h \cdot D_{t}^{3} h$, whose $L^{2}$ norm is bounded via Sobolev lemma (A.4). We have

$$
\begin{aligned}
& \left\|e^{\prime \prime}(h)\left(D_{t}^{3} h\right)^{2}\right\|_{L^{2}(\Omega)} \lesssim_{c_{0}}\left\|\left(e^{\prime}(h) D_{t}^{3} h\right)^{2}\right\|_{L^{2}(\Omega)} \lesssim_{K, c_{0}}\left(\sum_{j=0,1}\left\|\nabla^{j}\left(e^{\prime}(h) D_{t}^{3} h\right)\right\|_{L^{2}(\Omega)}\right)^{2} \\
& \lesssim_{K, M, c_{0}}\left(\left|e^{\prime}(h)\right| \cdot|| \nabla D_{t}^{3} h\left\|_{L^{2}(\Omega)}+\right\| e^{\prime}(h) D_{t}^{3} h \|_{L^{2}(\Omega)}\right)^{2}
\end{aligned}
$$

Hence we conclude

$$
\left\|g_{5}\right\|_{L^{2}(\Omega)} \lesssim K, M, c_{0} \sum_{j \leq 5}\left\|e^{\prime}(h) D_{t}^{j} h\right\|_{L^{2}(\Omega)}+\left(\left|e^{\prime}(h)\right| \cdot\left\|\nabla D_{t}^{3} h\right\|_{L^{2}(\Omega)}+\left\|e^{\prime}(h) D_{t}^{3} h\right\|_{L^{2}(\Omega)}\right)^{2} .
$$




\subsection{When $r \geq 6$}

The estimates for the general case in fact follow from the case when $r=5$. Since $g_{r}$ is a sum of the products of the form (4.26), we apply the derivative counting method again on estimating each of the products.

- If $j_{m} \geq r-2$, then the product consists of at most 4 terms, where $j_{i}<r-2$ for all $i<m$, among which at least one must be of order no more than 2, i.e., they are of the form $D_{t}^{j_{l}} h$ with $j_{l} \leq 2$ (and so $e^{\prime}(h) D_{t}^{j_{l}} h$ satisfies (1.29) $)$. Hence, by Sobolev lemma (4.22),

$$
\begin{aligned}
& \left\|e^{(m)}(h) D_{t}^{j_{1}} h \cdots D_{t}^{j_{m}} h\right\|_{L^{2}(\Omega)} \lesssim c_{0}\left\|\left(e^{\prime}(h) D_{t}^{j_{1}} h\right) \cdots\left(e^{\prime}(h) D_{t}^{j_{m}} h\right)\right\|_{L^{2}(\Omega)} \\
& \quad \leq C_{r}\left(K, M, c_{0}, \sum_{k \leq r-2}\left\|D_{t} h\right\|_{k}\right) \sum_{k \leq r-1}\left\|D_{t} h\right\|_{k} .
\end{aligned}
$$

- If $j_{m}<r-3$, then by (4.24) we have

$$
\left\|e^{(m)}(h) D_{t}^{j_{1}} h \cdots D_{t}^{j_{m}} h\right\|_{L^{2}(\Omega)} \leq C_{r}\left(K, M, c_{0}, \sum_{k \leq r-2}\left\|D_{t} h\right\|_{k}\right) .
$$

- If $j_{m}=r-3$, then there exists at most one $j_{l}$, where $l<m$ such that $j_{l}=r-3$, and the product consists of at most 3 terms if this is the case. Hence, (4.30) holds by Sobolev lemma (4.22).

Therefore, one concludes that when $r \geq 6$,

$$
\left\|g_{r}\right\|_{L^{2}(\Omega)} \leq C_{r}\left(K, M, c_{0}, \sum_{k \leq r-2}\left\|D_{t} h\right\|_{k}\right) \sum_{k \leq r-1}\left\|D_{t} h\right\|_{k},
$$

where $C_{r}$ are continuous functions.

In summary, we have proved:

Theorem 4.2. Let $f_{r}$ and $g_{r}$ be defined as (4.12) and (4.26), respectively. Then we have the estimates

$$
\begin{gathered}
\left\|f_{r}\right\|_{L^{2}(\Omega)} \leq C(M)\left(\left\|\nabla^{r} v\right\|_{L^{2}(\Omega)}+\left\|\nabla^{r} h\right\|_{L^{2}(\Omega)}\right), \quad r=1,2 \\
\left\|f_{r}\right\|_{L^{2}(\Omega)} \leq C_{r}\left(K, M, \sum_{k \leq r-2}\left\|\nabla^{k} v\right\|_{L^{2}(\Omega)}, \sum_{k \leq r-2}\left\|D_{t} h\right\|_{k, 0}\right) \cdot \\
\left(\sum_{k \leq r-1}\left\|\nabla^{k} v\right\|_{L^{2}(\Omega)}+\sum_{k \leq r-1}\|h\|_{k, 0}+\sum_{k \leq r-1}\left\|D_{t} h\right\|_{k, 0}\right), \quad 3 \leq r \leq 5 \\
\left\|f_{r}\right\|_{L^{2}(\Omega)} \leq C_{r}\left(K, M, \sum_{k \leq r-2}\left\|\nabla^{k} v\right\|_{L^{2}(\Omega)}, \sum_{k \leq r-2}\|h\|_{k, 0}\right) \cdot \\
\left(\sum_{k \leq r-1}\left\|\nabla^{k} v\right\|_{L^{2}(\Omega)}+\sum_{k \leq r-1}\|h\|_{k, 0}+\sum_{k \leq r-1}\left\|D_{t} h\right\|_{k, 0}\right), \quad r \geq 6
\end{gathered}
$$

and

$$
\begin{array}{r}
\left\|g_{r}\right\|_{L^{2}(\Omega)} \leq C\left(M, c_{0}\right) \sum_{j \leq r}\left\|\sqrt{e^{\prime}(h)} D_{t}^{j} h\right\|_{L^{2}(\Omega)}, \quad 1 \leq r \leq 4 \\
\left\|g_{r}\right\|_{L^{2}(\Omega)} \leq C_{r}\left(K, M, c_{0}, \sum_{k \leq r-2}\left\|D_{t} h\right\|_{k}\right) \sum_{k \leq r-1}\left\|D_{t} h\right\|_{k}, \quad r \geq 5
\end{array}
$$




\subsection{Improved estimates for $\left\|f_{r}\right\|_{L^{2}(\Omega)}$ and $\left\|g_{r}\right\|_{L^{2}(\Omega)}$}

Definition 4.3. (Improved mixed norms)

- $\|h\|_{r, 1,0}:=\sum_{k+s=r, k<r-1}\left\|\nabla^{s} D_{t}^{k} h\right\|_{L^{2}(\Omega)}+\left\|\sqrt{e^{\prime}(h)} \nabla D_{t}^{r-1} h\right\|_{L^{2}(\Omega)}$,

- $\|h\|_{r, 1}:=\|h\|_{r, 1,0}+\left\|e^{\prime}(h) D_{t}^{r} h\right\|_{L^{2}(\Omega)}$.

Under these new norms, the estimates for $\left\|f_{r}\right\|_{L^{2}(\Omega)}$ and $\left\|g_{r}\right\|_{L^{2}(\Omega)}$ can be improved as:

Theorem 4.3. Let $f_{r}$ and $g_{r}$ be defined as (4.12) and (4.26), respectively. Then,

$$
\begin{aligned}
& \left\|f_{r}\right\|_{L^{2}(\Omega)} \leq C_{r}\left(K, M, \sum_{k \leq r-2}\left\|\nabla^{k} v\right\|_{L^{2}(\Omega)}, \sum_{k \leq r-2}\left\|D_{t} h\right\|_{k, 0}\right) . \\
& \left(\sum_{k \leq r-1}\left\|\nabla^{k} v\right\|_{L^{2}(\Omega)}+\sum_{k \leq r-1}\|h\|_{k, 0}+\sum_{k \leq r-1}\left\|D_{t} h\right\|_{k, 1,0}\right), \quad 3 \leq r \leq 5 \\
& \left\|f_{r}\right\|_{L^{2}(\Omega)} \leq C_{r}\left(K, M, \sum_{k \leq r-2}\left\|\nabla^{k} v\right\|_{L^{2}(\Omega)}, \sum_{k \leq r-2}\|h\|_{k, 0}\right) . \\
& \quad\left(\sum_{k \leq r-1}\left\|\nabla^{k} v\right\|_{L^{2}(\Omega)}+\sum_{k \leq r-1}\|h\|_{k, 0}+\sum_{k \leq r-1}\left\|D_{t} h\right\|_{k, 1,0}\right), \quad r \geq 6
\end{aligned}
$$

and

$$
\begin{array}{r}
\left\|g_{r}\right\|_{L^{2}(\Omega)} \leq C\left(M, c_{0}\right) \sum_{j \leq r}\left\|e^{\prime}(h) D_{t}^{j} h\right\|_{L^{2}(\Omega)}, \quad 1 \leq r \leq 4 \\
\left\|g_{r}\right\|_{L^{2}(\Omega)} \leq C_{r}\left(K, M, c_{0}, \sum_{k \leq r-2}\left\|D_{t} h\right\|_{k, 1}\right) \sum_{k \leq r-1}\left\|D_{t} h\right\|_{k, 1}, \quad r \geq 5
\end{array}
$$

Proof. It is easy to observe that the estimates for $\left\|f_{r}\right\|_{L^{2}(\Omega)}$ and $\left\|g_{r}\right\|_{L^{2}(\Omega)}$ does not include the quantity $\left\|\nabla D_{t}^{r-1} h\right\|_{L^{2}(\Omega)}$, and we no longer use $e^{\prime}(h) \leq c_{0} \sqrt{e^{\prime}(h)} \leq c_{0}$ in the estimates for $\left\|g_{r}\right\|_{L^{2}(\Omega)}$; in other words, we keep $e^{\prime}(h)$ whenever it is possible.

Theorem 4.3 is essential for estimating the lower order terms $\left\|\nabla D_{t}^{k} h\right\|_{L^{2}(\Omega)}, 0 \leq k \leq r-1$ without using the wave equation (See Section 6).

\section{Energy estimates for Euler equations with free boundary}

Proposition 5.1. Let $E_{r}$ be defined as (1.12), then there are continuous functions $C_{r}$ such that, for $t \in[0, T]$,

$$
\left|\frac{d E_{r}(t)}{d t}\right| \leq C_{r}\left(K, \frac{1}{\epsilon}, M, c_{0}, E_{r-1}^{*}\right) E_{r}^{*}(t)
$$

holds for all $r \geq 1$, where $E_{r}^{*}=\sum_{i \leq r} E_{i}$, provided the assumption (1.5) and the a priori bounds (1.24)-(1.29).

\subsection{Computing $\frac{d}{d t} E_{r}$}

Our computation for $\frac{d}{d t} E_{r}(t)$ is almost identical to what we had in [20, but we can no longer use the interpolation (A.6) which relies on the $L^{\infty}$ bounds of $\left|D_{t} h\right|$ and $\left|D_{t}^{2} h\right|$. We first compute $\frac{d}{d t} E_{s, k}$, where $E_{s, k}$ is defined as (1.13), when $s>0$.

$$
\begin{aligned}
& \frac{d}{d t} E_{s, k}=\frac{1}{2} \int_{\mathcal{D}_{t}} \rho D_{t}\left(\delta^{i j} Q\left(\partial^{s} D_{t}^{k} v_{i}, \partial^{s} D_{t}^{k} v_{j}\right) d x+\frac{1}{2} \int_{\mathcal{D}_{t}} \rho D_{t}\left(e^{\prime}(h) Q\left(\partial^{s} D_{t}^{k} h, \partial^{s} D_{t}^{k} h\right)\right) d x\right. \\
& \quad+\frac{1}{2} \int_{\partial \mathcal{D}_{t}} \rho D_{t}\left(Q\left(\partial^{s} D_{t}^{k} h, \partial^{s} D_{t}^{k} h\right) \nu\right)-Q\left(\partial^{s} D_{t}^{k} h, \partial^{s} D_{t}^{k} h\right) \nu(\sigma v \cdot N)+\rho Q\left(\partial^{s} D_{t}^{k} h, \partial^{s} D_{t}^{k} h\right) D_{t} \nu d S
\end{aligned}
$$


The estimates (A.1)-A.4 together with a priori assumptions imply

$$
\begin{gathered}
\left|D_{t} q^{i j}\right| \lesssim M, \quad\left|\partial q^{i j}\right| \lesssim M+K, \quad|\sigma v \cdot N|_{L^{\infty}(\partial \Omega)} \lesssim K+M \\
\left|D_{t} \nu\right|_{L^{\infty}(\partial \Omega)}=\left|D_{t}\left(-\nabla_{N} h\right)^{-1}\right|_{L^{\infty}(\partial \Omega)} \lesssim 1+\frac{1}{M}
\end{gathered}
$$

and

$$
D_{t} \gamma^{i j}=-2 \gamma^{i m} \gamma^{j n}\left(\frac{1}{2} D_{t} g_{m n}\right)
$$

Since $\left|D_{t} q^{i j}\right| \lesssim M$ in the interior and on the boundary $q^{i j}=\gamma^{i j}$, and by (5.3) $D_{t} \gamma$ is tangential, so that (5.2) can then be reduced to

$$
\begin{aligned}
\frac{d}{d t} E_{s, k} \leq \int_{\mathcal{D}_{t}} \rho \delta^{i j} Q\left(D_{t} \partial^{s} D_{t}^{k} v_{i}, \partial^{s} D_{t}^{k} v_{j}\right) d x & +\int_{\mathcal{D}_{t}} \rho e^{\prime}(h) Q\left(D_{t} \partial^{s} D_{t}^{k} h, \partial^{s} D_{t}^{k} h\right) d x \\
& \quad \int_{\partial \mathcal{D}_{t}} \rho Q\left(D_{t} \partial^{s} D_{t}^{k} h, \partial^{s} D_{t}^{k} h\right) \nu d S+C(K, M)\left(E_{r}+\|h\|_{j}^{2}+\|v\|_{r, 0}^{2}\right) .
\end{aligned}
$$

Now, if $s \geq 1$, our commutators (4.4) and (4.5) yield, since $D_{t} v_{i}=-\partial_{i} h-e_{n}$,

$$
\begin{array}{r}
D_{t} \partial^{s} D_{t}^{k} v_{i}=-\partial^{s} D_{t}^{k} \partial_{i} h+\sum_{0 \leq m \leq s-1} c_{s r}\left(\partial^{m+1} v\right) \cdot \partial^{s-m} D_{t}^{k} v_{i}, \\
D_{t} \partial^{r} h+\left(\partial_{j} h\right) \partial^{r} v^{j}=\partial^{r} D_{t} h+\sum_{0 \leq m \leq r-2} d_{s r}\left(\partial^{m+1} v\right) \sim \partial^{r-m} h, \\
D_{t} \partial^{s} D_{t}^{k} h=\partial^{s} D_{t}^{k+1} h+\sum_{0 \leq m \leq s-1} d_{s r}\left(\partial^{m+1} v\right): \partial^{s-m} D_{t}^{k} h, \quad \text { for } \quad k \geq 1 .
\end{array}
$$

We control the term $\left\|\left(\partial^{m+1} v\right) \sim \partial^{s-m} D_{t}^{k} v_{i}\right\|_{L^{2}\left(\mathcal{D}_{t}\right)}$ in (5.5) and $\left\|\left(\partial^{m+1} v\right) \sim \partial^{s-m} D_{t}^{k} h\right\|_{L^{2}\left(\mathcal{D}_{t}\right)}$ in (5.7) for $s+k=r$ and $s \geq 1$.

- The term $\left\|\left(\partial^{m+1} v\right) \widetilde{r} \partial^{s-m} D_{t}^{k} h\right\|_{L^{2}\left(\mathcal{D}_{t}\right)}$ can be bounded by

1. For $k=0$,

$$
\left\|\left(\partial^{m+1} v\right)^{\sim} \partial^{r-m} h\right\|_{L^{2}\left(\mathcal{D}_{t}\right)} \lesssim K|\partial v|_{L^{\infty}} \sum_{j \leq r}\left\|\partial^{r} h\right\|_{L^{2}\left(\mathcal{D}_{t}\right)}+|\partial h|_{L^{\infty}} \sum_{j \leq r}\left\|\partial^{r} v\right\|_{L^{2}\left(\mathcal{D}_{t}\right)} .
$$

2. For $k=r-1$ (and so $m=0$ ),

$$
\left\|(\partial v)^{\sim}\left(\partial D_{t}^{r-1} h\right)\right\|_{L^{2}\left(\mathcal{D}_{t}\right)} \leq|\partial v|_{L^{\infty}}\left\|\partial D_{t}^{r-1} h\right\|_{L^{2}\left(\mathcal{D}_{t}\right)} .
$$

3. For $1 \leq k \leq r-2$, if $m=0$ then

$$
\left\|(\partial v)^{\sim} \partial^{s} D_{t}^{k} h\right\|_{L^{2}\left(\mathcal{D}_{t}\right)} \leq|\partial v|_{L^{\infty}}\left\|\partial^{s} D_{t}^{k} h\right\|_{L^{2}\left(\mathcal{D}_{t}\right)} .
$$

On the other hand, if $m \geq 1$ and $r \geq 4$, we have

$$
\left\|\left(\partial^{m+1} v\right) \sim \partial^{s-m} D_{t}^{k} h\right\|_{L^{2}\left(\mathcal{D}_{t}\right)} \lesssim K \sum_{i=1,2}\left\|\partial^{m+i} v\right\|_{L^{2}\left(\mathcal{D}_{t}\right)} \cdot \sum_{j=0,1}\left\|\partial^{s-m+j} D_{t}^{k} h\right\|_{L^{2}\left(\mathcal{D}_{t}\right)} .
$$

Here, at most one of $m+2$ or $r-m+1$ can in fact equal to $r$ when $r \geq 4$. However, if $r=3$, then $k$ must equal to 1 , and so $s=2$. Hence,

\footnotetext{
${ }^{2}$ We refer Section 5 of [5] for the detailed proof
} 
- The term $\left\|\left(\partial^{m+1} v\right) \cdot \partial^{s-m} D_{t}^{k} v_{i}\right\|_{L^{2}\left(\mathcal{D}_{t}\right)}$ can be bounded similarly as above with $h$ replaced by $v$.

The above anaylsis shows that the $L^{2}$ norm of the sum in (5.5)-(5.7) contribute only to $\|v\|_{r, 0}$ and $\|h\|_{r, 0}$. Hence,

$$
\begin{gathered}
\frac{d}{d t} E_{r} \leq-\int_{\mathcal{D}_{t}} \rho\left(\delta^{i j} Q\left(\partial^{s} D_{t}^{k} v_{i}, \partial^{s} D_{t}^{k} \partial_{j} h\right) d x+\int_{\mathcal{D}_{t}} \rho e^{\prime}(h) Q\left(\partial^{s} D_{t}^{k} h, \partial^{s} D_{t}^{k+1} h\right) d x\right. \\
\quad+\int_{\partial \mathcal{D}_{t}} \rho Q\left(\partial^{s} D_{t}^{k} h, D_{t} \partial^{s} D_{t}^{k} h\right) \nu d S \\
+C(K, M)\left(\|v\|_{r, 0}+\|h\|_{r, 0}\right)\left(\sum_{i \leq r-1}\|v\|_{i, 0}+\|h\|_{i, 0}\right)\left(\sum_{i \leq r}\|v\|_{i, 0}+\|h\|_{i, 0}\right) .
\end{gathered}
$$

In addition, (4.5) and (4.19) yield that for $s+k=r$

$$
\begin{aligned}
& \left\|\partial^{s} D_{t}^{k} \partial h-\partial^{s+1} D_{t}^{k} h\right\|_{L^{2}\left(\mathcal{D}_{t}\right)} \lesssim \sum_{l_{1}+l_{2}=k-1}\left\|\partial^{s}\left(\partial D_{t}^{l_{1}} v^{\tilde{r}} \partial D_{t}^{l_{2}} h\right)\right\|_{L^{2}\left(\mathcal{D}_{t}\right)} \\
& +\sum_{l_{1}+\cdots+l_{n}=k-n+1, n \geq 3}\left\|\partial^{s}\left(\partial D_{t}^{l_{1}} v \cdots \partial D_{t}^{l_{n-1}} v^{\sim} \partial D_{t}^{l_{n}} h\right)\right\|_{L^{2}\left(\mathcal{D}_{t}\right)} \\
& \lesssim_{K, M}\left(\sum_{i \leq r-1}\|v\|_{i, 0}+\|h\|_{i, 0}\right)\left(\sum_{i \leq r}\|v\|_{i, 0}+\|h\|_{i, 0}\right) .
\end{aligned}
$$

Therefore,

$$
\begin{aligned}
& \frac{d}{d t} E_{r} \leq \int_{\mathcal{D}_{t}} \rho\left(\delta^{i j} Q\left(\partial^{s} D_{t}^{k} v_{i}, \partial_{j} \partial^{s} D_{t}^{k} h\right) d x\right.+\int_{\mathcal{D}_{t}} \rho e^{\prime}(h) Q\left(\partial^{s} D_{t}^{k} h, \partial^{s} D_{t}^{k+1} h\right) d x \\
&+\int_{\partial \mathcal{D}_{t}} \rho Q\left(\partial^{s} D_{t}^{k} h, D_{t} \partial^{s} D_{t}^{k} h\right) \nu d S \\
&+C(K, M)\left(\|v\|_{r, 0}+\|h\|_{r, 0}\right)\left(\sum_{i \leq r-1}\|v\|_{i, 0}+\|h\|_{i, 0}\right)\left(\sum_{i \leq r}\|v\|_{i, 0}+\|h\|_{i, 0}\right) .
\end{aligned}
$$

If we integrate by parts in the first term

$$
\begin{aligned}
\int_{\mathcal{D}_{t}} \rho \delta^{i j} Q\left(\partial^{s} D_{t}^{k} \partial_{i} v_{j}, \partial^{s} D_{t}^{k} h\right) & d x+\int_{\mathcal{D}_{t}} \rho e^{\prime}(h) Q\left(\partial^{s} D_{t}^{k} h, \partial^{s} D_{t}^{k+1} h\right) d x \\
& +\int_{\partial \mathcal{D}_{t}} \rho Q\left(\partial^{s} D_{t}^{k} h, D_{t} \partial^{s} D_{t}^{k} h-\nu^{-1} N_{i} \partial^{s} D_{t}^{k} v^{i}\right) \nu d S \\
& +C(K, M)\left(\|v\|_{r, 0}+\|h\|_{r, 0}\right)\left(\sum_{i \leq r-1}\|v\|_{i, 0}+\|h\|_{i, 0}\right)\left(\sum_{i \leq r}\|v\|_{i, 0}+\|h\|_{i, 0}\right) .
\end{aligned}
$$

But since $\partial^{s} D_{t}^{k+1} e(h)$ equals $e^{\prime}(h) \partial^{s} D_{t}^{k+1} h$ plus a sum of terms of the form

$$
e^{(m)}(h)\left(\partial^{i_{1}} D_{t}^{j_{1}} h\right) \cdots\left(\partial^{i_{m}} D_{t}^{j_{m}} h\right),
$$

where

$$
\left(i_{1}+j_{1}\right)+\cdots+\left(i_{m}+j_{m}\right) \leq r+1, \quad 1 \leq i_{1}+j_{1} \leq \cdots \leq i_{m}+j_{m} \leq r
$$

Therefore,

$$
\begin{gathered}
\int_{\mathcal{D}_{t}} \rho \delta^{i j} Q\left(\partial^{s} D_{t}^{k} \partial_{i} v_{j}, \partial^{s} D_{t}^{k} h\right) d x=\int_{\mathcal{D}_{t}} \rho Q\left(\partial^{s} D_{t}^{k} \operatorname{div} v, \partial^{s} D_{t}^{k} h\right) d x \\
=-\int_{\mathcal{D}_{t}} \rho Q\left(\partial^{s} D_{t}^{k+1} e(h), \partial^{s} D_{t}^{k} h\right) d x \leq-\int_{\mathcal{D}_{t}} \rho e^{\prime}(h) Q\left(\partial^{s} D_{t}^{k+1} h, \partial^{s} D_{t}^{k} h\right) d x \\
\quad+C(K, M)\left(\|v\|_{r, 0}+\|h\|_{r, 0}\right)\left(\sum_{i \leq r-1}\|v\|_{i, 0}+\|h\|_{i, 0}\right)\left(\sum_{i \leq r}\|v\|_{i, 0}+\|h\|_{i, 0}\right),
\end{gathered}
$$


so the first integral in (5.11) cancels with the second term.

We recall $\nu=-\left(\partial_{N} h\right)^{-1}$, so that $\nu^{-1} N_{i}=\partial_{i} h$. Hence, the boundary term in (5.11) becomes

$$
\sum_{k+s=r, s>0} \int_{\partial \mathcal{D}_{t}} \rho Q\left(\partial^{s} D_{t}^{k} h, D_{t} \partial^{s} D_{t}^{k} h+\left(\partial_{i} h\right)\left(\partial^{s} D_{t}^{k} v^{i}\right) \nu d S\right.
$$

Now, since (5.6) and (5.7), (5.13) becomes sum of the boundary inner product of $\Pi \partial^{s} D_{t}^{k} h$ and

$$
\begin{array}{r}
\Pi\left(D_{t} \partial^{r} h+\left(\partial_{j} h\right) \partial^{r} v^{j}\right)=\Pi \partial^{r} D_{t} h+\sum_{0 \leq m \leq r-2} d_{m r} \Pi\left(\left(\partial^{m+1} v\right) \sim \partial^{r-m} h\right), \\
\Pi\left(D_{t} \partial^{s} D_{t}^{k} h+\left(\partial_{i} h\right)\left(\partial^{s} D_{t}^{k} v^{i}\right)\right)=\Pi \partial^{s} D_{t}^{k+1} h+\Pi\left(\partial_{i} h\right)\left(\partial^{s} D_{t}^{k} v^{i}\right)+\sum_{0 \leq m \leq s-1} d_{m r} \Pi\left(\left(\partial^{m+1} v\right): \partial^{s-m} D_{t}^{k} h\right),
\end{array}
$$

for $k=0$ and $k>0$, respectively.

In addition, when $s=0$,

$$
\frac{d}{d t} E_{0, r} \leq-\int_{\mathcal{D}_{t}} \rho \delta^{i j}\left(D_{t}^{r} \partial_{i} h\right)\left(D_{t}^{r} v_{j}\right) d x+\int_{\mathcal{D}_{t}} \rho e^{\prime}(h)\left(D_{t}^{r+1} h\right)\left(D_{t}^{r} h\right) d x+C(M)\left\|e^{\prime}(h) D_{t}^{r} h\right\|_{L^{2}\left(\mathcal{D}_{t}\right)}^{2},
$$

where we have used the fact that $\left|e^{\prime \prime}(h)\right| \leq c_{0}\left|e^{\prime}(h)\right|$. Furthermore, since

$$
\begin{aligned}
& \left\|D_{t}^{r} \partial h-\partial D_{t}^{r} h\right\|_{L^{2}\left(\mathcal{D}_{t}\right)} \lesssim \sum_{l_{1}+l_{2}=r-1}\left\|\partial D_{t}^{l_{1}} v^{\sim} \partial D_{t}^{l_{2}} h\right\|_{L^{2}\left(\mathcal{D}_{t}\right)} \\
& +\sum_{l_{1}+\cdots+l_{n}=r-n+1, n \geq 3}\left\|\partial D_{t}^{l_{1}} v \cdots \partial D_{t}^{l_{n-1}} v^{\sim} \partial D_{t}^{l_{n}} h\right\|_{L^{2}\left(\mathcal{D}_{t}\right)} \lesssim K, M \\
& \left.\quad \sum_{i \leq r-1}\|v\|_{i, 0}+\|h\|_{i, 0}\right)\left(\sum_{i \leq r}\|v\|_{i, 0}+\|h\|_{i, 0}\right),
\end{aligned}
$$

(5.16) becomes, after integrating by parts on the first integral on the RHS of (5.16),

$$
\begin{aligned}
\frac{d}{d t} E_{0, r} \leq & \int_{\mathcal{D}_{t}} \rho \delta^{i j}\left(D_{t}^{r} h\right)\left(D_{t}^{r} \operatorname{div} v\right) d x+\int_{\mathcal{D}_{t}} \rho e^{\prime}(h)\left(D_{t}^{r+1} h\right)\left(D_{t}^{r} h\right) d x \\
& +C(M)\left\|e^{\prime}(h) D_{t}^{r} h\right\|_{L^{2}\left(\mathcal{D}_{t}\right)}^{2}+C(M) \sum_{i \leq r}\left(\|h\|_{i, 0}+\|v\|_{i, 0}\right)^{2} .
\end{aligned}
$$

But since

$$
D_{t}^{r} \operatorname{div} v=-D_{t}^{r+1} e(h)=-e^{\prime}(h) D_{t}^{r+1} h-g_{r},
$$

and because $\left\|e^{\prime}(h) D_{t}^{r} h\right\|_{L^{2}\left(\mathcal{D}_{t}\right)} \leq c_{0}\left\|\sqrt{e^{\prime}(h)} D_{t}^{r} h\right\|_{L^{2}\left(\mathcal{D}_{t}\right)}$, which is part of $\|h\|_{r}$, (5.18) becomes

$$
\frac{d}{d t} E_{0, r} \leq C(M) \sum_{i \leq r}\left(\|h\|_{i}+\|v\|_{i, 0}\right)^{2} .
$$

Furthermore, let $K_{r}$ be defined as (1.14), we have

$$
\frac{d}{d t} K_{r}=2 \int_{\mathcal{D}_{t}} \rho\left|\partial^{r-1} \operatorname{curl} v\right| \cdot\left|D_{t} \partial^{r-1} \operatorname{curl} v\right| d x
$$

But since the curl satisfies the equation

$$
D_{t} \operatorname{curl}_{i j} v=-\left(\partial_{i} v^{k}\right)\left(\operatorname{curl}_{k j} v\right)+\left(\partial_{j} v^{k}\right)\left(\operatorname{curl}_{k i} v\right),
$$


then

$$
\begin{aligned}
& \left|D_{t} \partial^{r-1} \operatorname{curl} v\right| \leq\left|\partial^{r-1} D_{t} \operatorname{curl} v\right|+\sum_{0 \leq m \leq r-2} e_{m r}\left(\partial^{m+1} v\right): \partial^{r-1-m} \operatorname{curl} v \\
& \quad \lesssim \sum_{0 \leq m \leq r-1} e_{m r}\left(\partial^{m+1} v\right): \partial^{r-1-m} \operatorname{curl} v .
\end{aligned}
$$

The term $\left.\|\left(\partial^{m+1} v\right)\right)^{r} \partial^{r-1-m} \operatorname{curl} v \|_{L^{2}\left(\mathcal{D}_{t}\right)}$ can be bounded by

$$
|\partial v|_{L^{\infty}} \sum_{j \leq r-1}\left\|\partial^{j} \operatorname{curl} v\right\|_{L^{2}\left(\mathcal{D}_{t}\right)}+|\operatorname{curl} v|_{L^{\infty}} \sum_{j \leq r-1}\left\|\partial^{j+1} v\right\|_{L^{2}\left(\mathcal{D}_{t}\right)} .
$$

On the other hand,

$$
\begin{aligned}
& \sum_{j \leq r+1} \frac{d W_{j}^{2}}{d t} \lesssim \sum_{j \leq r+1}\left(W_{j}^{2}+W_{j}\left(\left\|f_{j}\right\|_{L^{2}\left(\mathcal{D}_{t}\right)}+\left\|g_{j}\right\|_{L^{2}\left(\mathcal{D}_{t}\right)}\right)\right. \\
& \lesssim E_{r}^{*}+\sum_{j \leq r}\left(\left\|f_{r}\right\|_{L^{2}\left(\mathcal{D}_{t}\right)}^{2}+\left\|g_{r}\right\|_{L^{2}\left(\mathcal{D}_{t}\right)}^{2}\right) .
\end{aligned}
$$

The first inequality comes from the energy estimates for the wave equation, e.g., Theorem 4.1

Summing these up, we have proved:

Theorem 5.2. Let $E_{r}$ be defined as (1.12), for all $r \geq 1$ we have

$$
\begin{aligned}
\left|\frac{d E_{r}}{d t}\right| \lesssim_{\Sigma, M} E_{r}^{*} & +\sum_{k+s=r, k, s>0}\left(\| \Pi \partial ^ { s } D _ { t } ^ { k } h \| _ { L ^ { 2 } ( \partial \mathcal { D } _ { t } ) } \left(\left\|\Pi \partial^{s} D_{t}^{k+1} h\right\|_{L^{2}\left(\partial \mathcal{D}_{t}\right)}\right.\right. \\
& \left.\left.+\left\|\Pi\left(\partial_{i} h\right)\left(\partial^{s} D_{t}^{k} v^{i}\right)\right\|_{L^{2}\left(\partial \mathcal{D}_{t}\right)}+\sum_{0 \leq m \leq s-1}\left\|\Pi\left(\left(\partial^{m+1} v\right) \partial^{s-m} D_{t}^{k} h\right)\right\|_{L^{2}\left(\partial \mathcal{D}_{t}\right)}\right)\right) \\
+ & \left\|\Pi \partial^{r} h\right\|_{L^{2}\left(\partial \mathcal{D}_{t}\right)}\left(\left\|\Pi \partial^{r} D_{t} h\right\|_{L^{2}\left(\partial \mathcal{D}_{t}\right)}+\sum_{0 \leq m \leq r-2}\left\|\Pi\left(\left(\partial^{m+1} v\right) \partial^{r-m} h\right)\right\|_{L^{2}\left(\partial \mathcal{D}_{t}\right)}\right) \\
& +C(K, M)\left(\sum_{i \leq r-1}\|v\|_{i, 0}+\|h\|_{i, 0}\right)\left(\sum_{i \leq r}\|v\|_{i, 0}+\|h\|_{i}\right)^{2}+\sum_{j \leq r}\left(\left\|f_{r}\right\|_{L^{2}\left(\mathcal{D}_{t}\right)}^{2}+\left\|g_{r}\right\|_{L^{2}\left(\mathcal{D}_{t}\right)}^{2}\right)
\end{aligned}
$$

Definition 5.1. (Mixed boundary Sobolev norm) let $u(t, \cdot): \mathbb{R}^{n} \rightarrow \mathbb{R}$ be a smooth function. We define

$$
\langle\langle u\rangle\rangle_{r}=\sum_{k+s=r}\left\|\nabla^{s} D_{t}^{k} u\right\|_{L^{2}(\partial \Omega)} .
$$

Now, let us get back to Lagrangian coordinate. Based on the computation we have as well as A.20), controlling $\frac{d}{d t}$ requires to bound

$$
\|v\|_{r, 0},\|h\|_{r}, \sum_{j \leq r-1}\left\|\nabla^{j} v\right\|_{L^{2}(\partial \Omega)},\langle\langle h\rangle\rangle_{r}
$$

and

$$
\sum_{k+s=r, s \geq 2}\left\|\Pi \nabla^{s} D_{t}^{k+1} h\right\|_{L^{2}(\partial \Omega)} .
$$

Theorem 5.3. With the a priori assumptions (1.24)-(1.29) hold, there are continuous functions $C_{r}$ such that,

$$
\|v\|_{r, 0}^{2}+\|h\|_{r}^{2} \leq C_{r}\left(K, M, c_{0}, E_{r-1}^{*}\right) E_{r}^{*}
$$

In addition to that,

$$
\left\|D_{t} h\right\|_{r, 1}^{2}+\langle\langle h\rangle\rangle_{r}^{2} \leq C_{r}\left(K, M, c_{0}, \frac{1}{\epsilon}, E_{r-1}^{*}\right) E_{r}^{*},
$$

where $\left\|D_{t} h\right\|_{r, 1}$ is given in Definition 4.3 . 


\subsection{Interior estimates, bounds for $\|v\|_{r, 0},\|h\|_{r}$}

Our strategy is to first apply Theorem 3.1 to control $\|v\|_{r, 0}$ in terms of the energies $E_{r}$ and $L^{2}$ norm of $h$, and then we will apply our elliptic estimate (3.4) to control $\|h\|_{r}$. Now, since

$$
\|v\|_{r, 0} \leq\left\|\nabla^{r} v\right\|_{L^{2}(\Omega)}+\sum_{k+s=r, 0<k<r}\left\|\nabla^{s} D_{t}^{k} v\right\|_{L^{2}(\Omega)},
$$

and (4.19) yields

$$
\nabla^{s} D_{t}^{k} v=-\nabla^{s+1} D_{t}^{k-1} h+c_{\alpha \beta \gamma}\left(\partial^{\alpha_{1}} v\right) \cdots\left(\partial^{\alpha_{m}} v\right)\left(\partial^{\beta_{1}} D_{t}^{\gamma_{1}} h\right) \cdots\left(\partial^{\beta_{n}} D_{t}^{\gamma_{n}} h\right),
$$

where $3^{3}$

$$
\begin{gathered}
\alpha_{1}+\cdots+\alpha_{m}+\left(\beta_{1}+\gamma_{1}\right)+\cdots+\left(\beta_{n}+\gamma_{n}\right)=r, \\
1 \leq \alpha_{i} \leq r-1, \quad 1 \leq \beta_{j}+\gamma_{j} \leq r-1 .
\end{gathered}
$$

This implies that 4

$$
\begin{aligned}
& \sum_{k+s=r, 0<k<r}\left\|\nabla^{s} D_{t}^{k} v\right\|_{L^{2}(\Omega)} \leq \sum_{k+s=r, 0<k<r}\left\|\nabla^{s+1} D_{t}^{k-1} h\right\|_{L^{2}(\Omega)} \\
& +C_{r}\left(K, M, \sum_{j \leq r-2}\left\|\nabla^{j} v\right\|_{L^{2}(\Omega)}, \sum_{j \leq r-2}\|h\|_{j, 0}\right)\left(\sum_{j \leq r-1}\left\|\nabla^{j} v\right\|_{L^{2}(\Omega)}+\sum_{j \leq r-1}\|h\|_{j, 0}\right) .
\end{aligned}
$$

So the terms of order $r$ except for $\left\|\nabla^{r} v\right\|$ can be combined with $\|h\|_{r}$, up to lower order terms. Now, Theorem 3.1 yields,

$$
\left\|\nabla^{r} v\right\|_{L^{2}(\Omega)} \lesssim \sqrt{E_{r}}+\left\|\nabla^{r-1} \operatorname{div} v\right\|_{L^{2}(\Omega)} .
$$

We recall that $\operatorname{div} v=-e^{\prime}(h) D_{t} h$, hence

$$
\left\|\nabla^{r} v\right\|_{L^{2}(\Omega)} \lesssim_{M, c_{0}} \sqrt{E_{r}}+\sum_{j \leq r}\|h\|_{j, 0}
$$

via interpolation. Therefore,

$$
\begin{aligned}
& \|v\|_{r, 0} \lesssim K, M, c_{0} \sqrt{E_{r}}+\sum_{j \leq r}\|h\|_{j, 0} \\
& \quad+C_{r}\left(\sum_{j \leq r-2}\left\|\nabla^{j} v\right\|_{L^{2}(\Omega)}, \sum_{j \leq r-2}\|h\|_{j, 0}\right) \cdot\left(\sum_{j \leq r-1}\left\|\nabla^{j} v\right\|_{L^{2}(\Omega)}+\sum_{j \leq r-1}\|h\|_{j, 0}\right) .
\end{aligned}
$$

To bound $\|h\|_{r}$, since (3.4) provides, for each $k, s$ that $k+s=r$,

$$
\left\|\nabla^{s} D_{t}^{k} h\right\|_{L^{2}(\Omega)} \lesssim K, M\left\|\Pi \nabla^{s} D_{t}^{k} h\right\|_{L^{2}(\partial \Omega)}+\sum_{0 \leq j \leq s-2}\left\|\nabla^{j} \Delta D_{t}^{k} h\right\|_{L^{2}(\Omega)}+\left\|\nabla D_{t}^{k} h\right\|_{L^{2}(\Omega)},
$$

for $s \geq 2$. The term $\left\|\Pi \nabla^{s} D_{t}^{k} h\right\|_{L^{2}(\partial \Omega)}$ bounded by $\left(\|\nabla h\|_{L^{\infty}(\partial \Omega)} E_{r}\right)^{\frac{1}{2}}$, by the construction of $E_{r}$, whereas $\left\|\nabla D_{t}^{k} h\right\|_{L^{2}(\Omega)}$ is part of $\sum_{j=1}^{r} W_{j}$ since $k<r$. Further, by the wave equation (4.7),

$$
\sum_{\substack{0 \leq j \leq s-2 \\ 2 \leq s \leq r \\ s+k=r}}\left\|\nabla^{j} \Delta D_{t}^{k} h\right\|_{L^{2}(\Omega)} \leq \sum_{\substack{0 \leq j \leq s-2 \\ 2 \leq s \leq r \\ s+k=r}}\left(\left\|\nabla^{j} D_{t}^{k+2} e(h)\right\|_{L^{2}(\Omega)}+\left\|\nabla^{j} f_{k+1}\right\|_{L^{2}(\Omega)}+\left\|\nabla^{j} g_{k+1}\right\|_{L^{2}(\Omega)}\right) .
$$

\footnotetext{
${ }^{3}$ The second term on the right drops when $k=1$.

${ }^{4}$ We remark here that we have proved in 20 that if $r \leq 4$, then$$
\sum_{k+s=r, 0<k<r}\left\|\nabla^{s} D_{t}^{k} v\right\|_{L^{2}(\Omega)} \leq \sum_{k+s=r, 0<k<r}\left\|\nabla^{s+1} D_{t}^{k-1} h\right\|_{L^{2}(\Omega)}+C(K, M)\left(\sum_{j \leq r-1}\left\|\nabla^{j} v\right\|_{L^{2}(\Omega)}+\sum_{j \leq r-1}\|h\|_{j, 0}\right) .
$$ 
But since

$$
\nabla^{s-2} f_{k+1}=\sum_{\substack{\alpha_{1}+\cdots+\alpha_{m}+\left(\beta_{1}+\gamma_{1}\right)+\cdots+\left(\beta_{n}+\gamma_{n}\right)=r, 1 \leq \alpha_{i} \leq r-1,1 \leq \beta_{j} \leq r-1}} c_{\alpha \beta \gamma}\left(\partial^{\alpha_{1}} v\right) \cdots\left(\partial^{\alpha_{m}} v\right)\left(\partial^{\beta_{1}} D_{t}^{\gamma_{1}} h\right) \cdots\left(\partial^{\beta_{n}} D_{t}^{\gamma_{n}} h\right),
$$

and

$$
\nabla^{s-2} g_{k+1}=\sum_{\substack{\left(\alpha_{1}+\beta_{1}\right)+\cdots+\left(\alpha_{n}+\beta_{n}\right)=r \\ 1 \leq \alpha_{i}+\beta_{i} \leq r-1, n \geq 2}} c_{n \alpha \beta \gamma} e^{(n)}(h)\left(\partial^{\alpha_{1}} D_{t}^{\beta_{1}} h\right) \cdots\left(\partial^{\alpha_{n}} D_{t}^{\beta_{n}} h\right) .
$$

Thus,

$$
\begin{aligned}
& \sum_{j \leq s-2, s+k=r}\left(\left\|\nabla^{j} f_{k+1}\right\|_{L^{2}(\Omega)}+\left\|\nabla^{j} g_{k+1}\right\|_{L^{2}(\Omega)}\right) \leq \\
& C_{r}\left(K, M, \sum_{j \leq r-2}\left\|\nabla^{j} v\right\|_{L^{2}(\Omega)}, \sum_{j \leq r-2}\|h\|_{j}\right)\left(\sum_{j \leq r-1}\left\|\nabla^{j} v\right\|_{L^{2}(\Omega)}+\sum_{j \leq r-1}\|h\|_{j}\right) .
\end{aligned}
$$

On the other hand, since $\left|e^{(l)}(h)\right| \leq c_{0}\left|e^{\prime}(h)\right|$, and

$$
\begin{aligned}
\nabla^{s-2} D_{t}^{k+2} e(h)=e^{\prime}(h) \nabla^{s-2} D_{t}^{k+2} h & \\
& +\sum_{\substack{\left(\alpha_{1}+\beta_{1}\right)+\cdots+\left(\alpha_{m}+\beta_{m}\right)=r \\
1 \leq \alpha_{i}+\beta_{i} \leq r-1, m \geq 2}} c_{m \alpha \beta \gamma} e^{(m)}(h)\left(\partial^{\alpha_{1}} D_{t}^{\beta_{1}} h\right) \cdots\left(\partial^{\alpha_{m}} D_{t}^{\beta_{m}} h\right), \\
&
\end{aligned}
$$

we have

$$
\left\|\nabla^{s-2} D_{t}^{k+2} e(h)\right\|_{L^{2}(\Omega)} \leq c_{0}\left|e^{\prime}(h)\right| \cdot\left\|\nabla^{s-2} D_{t}^{k+2} h\right\|_{L^{2}(\Omega)}+C_{r}\left(K, M, c_{0}, \sum_{j \leq r-2}\|h\|_{j}\right) \cdot \sum_{j \leq r-1}\|h\|_{j} .
$$

Now, (5.37) and (5.39) yield

$$
\begin{aligned}
& \sum_{\substack{0 \leq j \leq s-2 \\
2 \leq s \leq r \\
s+k=r}}\left\|\nabla^{j} \Delta D_{t}^{k} h\right\|_{L^{2}(\Omega)} \lesssim K, M, c_{0}\left|e^{\prime}(h)\right| \sum_{\substack{0 \leq j \leq s-2 \\
2 \leq s \leq r \\
s+k=r}}\left\|\nabla^{j} D_{t}^{k+2} h\right\|_{L^{2}(\Omega)} \\
& +C_{r}\left(\sum_{j \leq r-2}\left\|\nabla^{j} v\right\|_{L^{2}(\Omega)}, \sum_{j \leq r-2}\|h\|_{j}\right) \cdot\left(\sum_{j \leq r-1}\left\|\nabla^{j} v\right\|_{L^{2}(\Omega)}+\sum_{j \leq r-1}\|h\|_{j}\right)
\end{aligned}
$$

Furthermore, we apply (3.4) again with $q=D_{t}^{k+2} h$ if $s-2 \geq 2$, and then repeat the estimates (5.34)-(5.39), we get

$$
\|h\|_{r} \lesssim K, M, c_{0} \sqrt{E_{r}^{*}}+\sum_{j \leq r} W_{j}+C_{r}\left(\sum_{j \leq r-2}\left\|\nabla^{j} v\right\|_{L^{2}(\Omega)}, \sum_{j \leq r-2}\|h\|_{j}\right) \cdot\left(\sum_{j \leq r-1}\left\|\nabla^{j} v\right\|_{L^{2}(\Omega)}+\sum_{j \leq r-1}\|h\|_{j}\right) .
$$

But since the last term is of lower order, i.e., it can be bounded by $C_{r}\left(K, M, c_{0}, E_{r-2}^{*}\right) \sqrt{E_{r-1}^{*}}$, and so (5.25) follows.

\subsection{Boundary estimates, bounds for $\sum_{j \leq r-1}\left\|\nabla^{j} v\right\|_{L^{2}(\partial \Omega)},\langle\langle h\rangle\rangle_{r}$ and $\left\|\bar{\nabla}^{r-2} \theta\right\|_{L^{2}(\partial \Omega)}$}

The control of $\sum_{j<r-1}\left\|\nabla^{j} v\right\|_{L^{2}(\partial \Omega)}$ follows directly form the estimate of $\sum_{j<r}\left\|\nabla^{j} v\right\|_{L^{2}(\Omega)}$ by trace theorem (Theorem A.12) . On the other hand, we shall not estimate $\langle\langle h\rangle\rangle_{r}$ alone; instead, we estimat5 $\left\|D_{t} h\right\|_{r, 1}+\langle\langle h\rangle\rangle_{r}$ (Definition 4.3) by (3.4). This has to be done since we need to estimate $\left\|f_{r+1}\right\|_{L^{2}\left(\mathcal{D}_{t}\right)}$ and $\left\|g_{r+1}\right\|_{L^{2}\left(\mathcal{D}_{t}\right)}$ by $E_{r}$.

\footnotetext{
${ }^{5}$ The reason that we use the norm $\left\|D_{t} h\right\|_{r, 1}$ instead of $\|h\|_{r+1}$ is because the latter involves $\left\|\nabla^{r+1} h\right\|$ which, after applying the elliptic and tensor estimates, gives $\left\|\left(\bar{\nabla}^{r-1} \theta\right) \nabla_{N} h\right\|_{L^{2}(\partial \Omega)}$ but $\left\|\bar{\nabla}^{r-1} \theta\right\|_{L^{2}(\partial \Omega)}$ can only be controlled by $E_{r+1}$. On the other hand, we want to avoid the term $\left\|\nabla D_{t}^{r} h\right\|_{L^{2}(\Omega)}$ (this term can not be estimated by the method given in Section 61 ) as well, in order to pass our estimates to the incompressible limit in Section 6
} 
We estimate the mixed boundary $L^{2}$ norm $\langle\langle h\rangle\rangle_{r}$ by (3.3), we have

$$
\langle\langle h\rangle\rangle_{r} \lesssim K, M, c_{0} \sum_{k+s=r}\left\|\Pi \nabla^{s} D_{t}^{k} h\right\|_{L^{2}(\partial \Omega)}+\sum_{\substack{k+s=r \\ j \leq s-1}}\left\|\nabla^{j} \Delta D_{t}^{k} h\right\|_{L^{2}(\Omega)}+\sum_{j \leq r-1}\left\|\nabla D_{t}^{j} h\right\|_{L^{2}(\Omega)} .
$$

In addition, for $0<\delta<1$, we have

$$
\begin{aligned}
& \left\|D_{t} h\right\|_{r, 1} \lesssim K, M, c_{0} \delta \sum_{\substack{k+s=r \\
s \geq 2}}\left\|\Pi \nabla^{s} D_{t}^{k+1} h\right\|_{L^{2}(\partial \Omega)} \\
& +\delta^{-1}\left(\sum_{\substack{k+s=r \\
s \geq 2, j \leq s-2}}\left\|\nabla^{j} \Delta D_{t}^{k+1} h\right\|_{L^{2}(\Omega)}+W_{r+1}+\sum_{j \leq r-2}\left\|\nabla D_{t}^{j+1} h\right\|_{L^{2}(\Omega)}\right),
\end{aligned}
$$

via (3.4). Moreover,

$$
\begin{aligned}
& \sum_{\substack{k+s=r \\
s \geq 1, j \leq s-1}}\left\|\nabla^{j} \Delta D_{t}^{k} h\right\|_{L^{2}(\Omega)}+\sum_{\substack{k+s=r \\
s \geq 2, j \leq s-2}}\left\|\nabla^{j} \Delta D_{t}^{k+1} h\right\|_{L^{2}(\Omega)} \lesssim K, M, c_{0} \\
& \quad \delta \sum_{\substack{k+s=r \\
s \geq 2}}\left\|\Pi \nabla^{s} D_{t}^{k+1} h\right\|_{L^{2}(\partial \Omega)}+\delta^{-1} \sum_{j \leq r+1} W_{j} \\
& +C_{r}\left(\sum_{j \leq r-1}\left\|\nabla^{j} v\right\|_{L^{2}(\Omega)}, \sum_{j \leq r-1}\|h\|_{j}\right) \cdot\left(\sum_{j \leq r}\left\|\nabla^{j} v\right\|_{L^{2}(\Omega)}+\sum_{j \leq r}\|h\|_{j}\right) .
\end{aligned}
$$

This in fact follows from the analysis we had for (5.40). Therefore, by (5.25), together with (5.43), and since $\sum_{j \leq r+1} W_{j}$ is part of $\sqrt{E_{r}^{*}}$, we obtain

$$
\left\|D_{t} h\right\|_{r, 1}+\langle\langle h\rangle\rangle_{r} \lesssim_{K, M, c_{0}} \delta \sum_{\substack{k+s=r \\ s \geq 2}}\left\|\Pi \nabla^{s} D_{t}^{k+1} h\right\|_{L^{2}(\partial \Omega)}+\delta^{-1} C_{r}\left(K, M, c_{0}, E_{r-1}^{*}\right) \sqrt{E_{r}^{*}} .
$$

On the other hand, applying (3.8) to $\left\|\Pi \nabla^{s} D_{t}^{k+1} h\right\|_{L^{2}(\partial \Omega)}$ with $q=D_{t}^{k+1} h$, then for $s+k=r$ and $s \geq 2$, we have

$$
\begin{aligned}
& \delta\left\|\Pi \nabla^{s} D_{t}^{k+1} h\right\|_{L^{2}(\partial \Omega)} \lesssim \delta\left\|\left(\bar{\nabla}^{s-2} \theta\right) \nabla_{N} D_{t}^{k+1} h\right\|_{L^{2}(\partial \Omega)}+\delta \sum_{j \leq s-1}\left\|\nabla^{j} D_{t}^{k+1} h\right\|_{L^{2}(\partial \Omega)} \\
& +\delta\left(\|\theta\|_{L^{\infty}(\partial \Omega)}+\sum_{0 \leq l \leq s-2}\left\|\bar{\nabla}^{l} \theta\right\|_{L^{2}(\partial \Omega)}\right)\left(\sum_{0 \leq l \leq s-2}\left\|\nabla^{l} D_{t}^{k+1} h\right\|_{L^{2}(\partial \Omega)}\right) \\
& \quad+\delta\left(\|\theta\|_{L^{\infty}(\partial \Omega)}+\sum_{0 \leq l \leq s-3}\left\|\bar{\nabla}^{l} \theta\right\|_{L^{2}(\partial \Omega)}\right)\left(\sum_{0 \leq l \leq s-1}\left\|\nabla^{l} D_{t}^{k+1} h\right\|_{L^{2}(\partial \Omega)}\right) .
\end{aligned}
$$

Now, we assume inductively that (5.26) holds for lower orders 6, i.e.,

$$
\left\|D_{t} h\right\|_{r^{\prime}, 1}+\langle\langle h\rangle\rangle_{r^{\prime}} \leq C_{r^{\prime}}\left(K, M, c_{0}, \frac{1}{\epsilon}, E_{r^{\prime}-1}^{*}\right) \sqrt{E_{r^{\prime}}^{*}}
$$

whenever $r^{\prime} \leq r-1$. Then (3.9) yields that

$$
\sum_{2 \leq s \leq r}\left\|\bar{\nabla}^{s-2} \theta\right\|_{L^{2}(\partial \Omega)} \leq C_{r}\left(K, M, c_{0}, \frac{1}{\epsilon}, E_{r-1}^{*}\right) \sqrt{E_{r}^{*}}
$$

\footnotetext{
${ }^{6}$ In fact, we have proved in [20] that [5.26] holds for $r \leq 4$.
} 
This, together with (5.47) implies that

$$
\begin{aligned}
& \delta \sum_{s+k=r}\left\|\Pi \nabla^{s} D_{t}^{k+1} h\right\|_{L^{2}(\partial \Omega)} \lesssim \delta \sum_{\substack{s+k=r \\
s \geq 2}}\left\|\left(\bar{\nabla}^{s-2} \theta\right) \nabla_{N} D_{t}^{k+1} h\right\|_{L^{2}(\partial \Omega)} \\
& +\delta C_{r}\left(K, M, c_{0}, \frac{1}{\epsilon}, E_{r-1}^{*}\right) \cdot \sum_{j \leq r}\langle\langle h\rangle\rangle_{j}+C_{r}\left(K, M, c_{0}, \frac{1}{\epsilon}, E_{r-1}^{*}\right) \sqrt{E_{r}^{*}} .
\end{aligned}
$$

Now, since $2 \leq s \leq r$, we have

$$
\begin{gathered}
\delta \sum_{\substack{s+k=r \\
s \geq 2}}\left\|\left(\bar{\nabla}^{s-2} \theta\right) \nabla_{N} D_{t}^{k+1} h\right\|_{L^{2}(\partial \Omega) \lesssim K} \delta\|\theta\|_{L^{\infty}(\partial \Omega)}\left\|\nabla D_{t}^{r-1} h\right\|_{L^{2}(\partial \Omega)}+\delta\left\|\nabla D_{t} h\right\|_{L^{\infty}(\partial \Omega)}\left\|\bar{\nabla}^{r-2} \theta\right\|_{L^{2}(\partial \Omega)} \\
+\sum_{\substack{s+k=r \\
3 \leq s \leq r-1}} \delta\left\|\bar{\nabla}^{s-2} \theta\right\|_{L^{2}(\partial \Omega)}^{\frac{1}{2}}\left\|\nabla D_{t}^{k+1} h\right\|_{L^{2}(\partial \Omega)}^{\frac{1}{2}}\left\|\bar{\nabla}^{s-2} \theta\right\|_{H^{1}(\partial \Omega)}^{\frac{1}{2}}\left\|\nabla D_{t}^{k+1} h\right\|_{H^{1}(\partial \Omega)}^{\frac{1}{2}}, \quad(5.5
\end{gathered}
$$

via (A.20) when $\Omega \in \mathbb{R}^{3}$. Furthermore, when $\Omega \in \mathbb{R}^{2}$ we have

$$
\begin{aligned}
& \delta \sum_{\substack{s+k=r \\
3 \leq s \leq r-1}}\left\|\left(\bar{\nabla}^{s-2} \theta\right) \nabla_{N} D_{t}^{k+1} h\right\|_{L^{2}(\partial \Omega)} \leq \sum_{\substack{s+k=r \\
3 \leq s \leq r-1 \\
3 \leq s}} \delta\left\|\nabla D_{t}^{k+1} h\right\|_{L^{\infty}(\partial \Omega)}\left\|\bar{\nabla}^{s-2} \theta\right\|_{L^{2}(\partial \Omega)} \\
& \quad \lesssim K \sum_{\substack{s+k=r \\
3 \leq s \leq r-1}} \delta\left\|\nabla D_{t}^{k+1} h\right\|_{H^{1}(\partial \Omega)}\left\|\bar{\nabla}^{s-2} \theta\right\|_{L^{2}(\partial \Omega)} .
\end{aligned}
$$

Moreover, applying (5.48) to (5.50) and (5.51) implies that

$$
\begin{aligned}
& \delta \sum_{\substack{s+k=r \\
s \geq 2}}\left\|\left(\bar{\nabla}^{s-2} \theta\right) \nabla_{N} D_{t}^{k+1} h\right\|_{L^{2}(\partial \Omega)} \leq C_{r}\left(K, M, c_{0}, \frac{1}{\epsilon}, E_{r-1}^{*}\right) \sqrt{E_{r}^{*}} \\
& \quad+\delta C_{r}\left(K, M, c_{0}, \frac{1}{\epsilon}, E_{r-1}^{*}\right)\langle\langle h\rangle\rangle_{r} .
\end{aligned}
$$

Thus, (5.49) becomes

$$
\delta \sum_{s+k=r}\left\|\Pi \nabla^{s} D_{t}^{k+1} h\right\|_{L^{2}(\partial \Omega)} \leq C_{r}\left(K, M, c_{0}, \frac{1}{\epsilon}, E_{r-1}^{*}\right)\left(\sqrt{E_{r}^{*}}+\delta\langle\langle h\rangle\rangle_{r}\right) .
$$

Therefore,

$$
\left\|D_{t} h\right\|_{r, 1}+\langle\langle h\rangle\rangle_{r} \leq C_{r}\left(K, M, c_{0}, \frac{1}{\epsilon}, E_{r-1}^{*}\right)\left(\sqrt{E_{r}^{*}}+\delta\langle\langle h\rangle\rangle_{r}\right)
$$

where the term

$$
\delta C_{r}\left(K, M, c_{0}, \frac{1}{\epsilon}, E_{r-1}^{*}\right)\langle\langle h\rangle\rangle_{r}
$$

can be moved to the LHS when $\delta=\delta\left(K, M, c_{0}, \frac{1}{\epsilon}, E_{r-1}^{*}\right)$ is chosen sufficiently small, and so (5.26) follows.

\subsection{Bounds for $\sum_{k+s=r}\left\|\Pi \nabla^{s} D_{t}^{k+1} h\right\|_{L^{2}(\partial \Omega)}$}

This is in fact (5.53) with $\delta=1$. But since now (5.26) has been proved, we obtain

$$
\sum_{k+s=r}\left\|\Pi \nabla^{s} D_{t}^{k+1} h\right\|_{L^{2}(\partial \Omega)} \leq C_{r}\left(K, M, c_{0}, \frac{1}{\epsilon}, E_{r-1}^{*}\right) \sqrt{E_{r}^{*}}
$$




\subsection{Bounds for $\sum_{j \leq r+1} \frac{d W_{j}^{2}}{d t}$}

We recall that we have

$$
\sum_{j \leq r+1} \frac{d W_{j}^{2}}{d t} \lesssim E_{r}^{*}+\sum_{j \leq r+1} W_{j}\left(\left\|f_{j}\right\|_{L^{2}(\Omega)}+\left\|g_{j}\right\|_{L^{2}(\Omega)}\right) .
$$

Therefore, it suffices to bound $\sum_{j=1}^{r+1}|| f_{j} \|_{L^{2}(\Omega)}$ and $\sum_{j=1}^{r+1}\left\|g_{j}\right\|_{L^{2}(\Omega)}$. However, we have

$$
\left\|f_{r+1}\right\|_{L^{2}(\Omega)}+\left\|g_{r+1}\right\|_{L^{2}(\Omega)} \leq C_{r}\left(K, M, c_{0}, \frac{1}{\epsilon}, E_{r-1}^{*}\right) \sqrt{E_{r}^{*}}
$$

this is because

$$
\sum_{j \leq r}\left(\left\|\nabla^{j} v\right\|_{L^{2}(\Omega)}+\|h\|_{j}+\left\|D_{t} h\right\|_{j}\right) \leq C_{r}\left(K, M, c_{0}, \frac{1}{\epsilon}, E_{r-1}^{*}\right) \sqrt{E_{r}^{*}},
$$

as a consequence of Theorem 5.3 .

\subsection{The energy estimates}

We are now ready to prove Proposition [5.1. Since we have showed that our energies $E_{r}$ control the interior and boundary Sobolev norms of $v$ and $h$, the only thing left is to control the product of the projected tensors, i.e.,

$$
\begin{gathered}
\sum_{s+k=r, s>0}\left(\sum_{0 \leq m \leq s-1} \Pi\left(\left(\nabla^{m+1} v\right) \approx \nabla^{s-m} D_{t}^{k} h\right)\right), \quad \text { for } k>0 \\
\sum_{0 \leq m \leq r-2} \Pi\left(\left(\nabla^{m+1} v\right) \cdot \nabla^{r-m} h\right), \quad \text { for } k=0 \\
\sum_{s+k=r, s>0} \Pi\left((\nabla h) \approx\left(\nabla^{s} D_{t}^{k} v\right)\right) . \quad \text { for } k>0
\end{gathered}
$$

We cannot use interpolation (A.7) here since it only applies to tangential derivative $\bar{\nabla}$. Our strategy is to apply Gagliardo-Nirenberg inequality (i.e., A.20) to control terms that involving mixed derivatives]. By letting $\alpha=\nabla^{s-1} v$ in (A.21) we get

$$
\left\|\nabla^{s-1} v\right\|_{L^{2}(\partial \Omega)} \lesssim K \sum_{j \leq s}\left\|\nabla^{j} v\right\|_{L^{2}(\Omega)} .
$$

Now, when $\Omega \in \mathbb{R}^{3}$, each term of (5.58) is bounded as

- If $m=0$, then

$$
\left\|\Pi\left((\nabla v)^{\sim} \nabla^{s} D_{t}^{k} h\right)\right\|_{L^{2}(\partial \Omega)} \leq\|\nabla v\|_{L^{\infty}}\left\|\nabla^{s} D_{t}^{k} h\right\|_{L^{2}(\partial \Omega)} .
$$

- If $m \geq 1$, since $k \geq 1$, we must have $1 \leq m \leq r-2$. But if $m=r-2$, then $k=1$ and so $s=r-1$, hence

$$
\left\|\Pi\left(\left(\nabla^{r-1} v\right) \approx \nabla D_{t} h\right)\right\|_{L^{2}(\partial \Omega)} \leq\left\|\nabla D_{t} h\right\|_{L^{\infty}}\left\|\nabla^{r-1} v\right\|_{L^{2}(\partial \Omega)} .
$$

Otherwise, since $1 \leq m \leq r-3$, we have

$$
\begin{aligned}
& \left\|\Pi\left(\left(\nabla^{m+1} v\right): \nabla^{s-m} D_{t}^{k} h\right)\right\|_{L^{2}(\partial \Omega)} \lesssim K \\
& \left\|\nabla^{m+1} v\right\|_{L^{2}(\partial \Omega)}^{1 / 2}\left\|\nabla^{s-m} D_{t}^{k} h\right\|_{L^{2}(\partial \Omega)}^{1 / 2}\left\|\nabla^{m+1} v\right\|_{H^{1}(\partial \Omega)}^{1 / 2}\left\|\nabla^{s-m} D_{t}^{k} h\right\|_{H^{1}(\partial \Omega)}^{1 / 2} .
\end{aligned}
$$

\footnotetext{
${ }^{7}$ We want our estimates to be linear in the highest order. One can use Sobolev lemma only to control mixed Sobolev norms as well but the highest order energy would appear quadratically that way.
} 
On the other hand, if $\Omega \in \mathbb{R}^{2}$, then (5.63) can instead be bounded via Sobolev lemma, i.e.,

$$
\left\|\Pi\left(\left(\nabla^{m+1} v\right): \nabla^{s-m} D_{t}^{k} h\right)\right\|_{L^{2}(\partial \Omega)} \lesssim_{K}\left\|\nabla^{m+2} v\right\|_{L^{2}(\partial \Omega)}\left\|\nabla^{s-m} D_{t}^{k} h\right\|_{L^{2}(\partial \Omega)} .
$$

Therefore, the boundary estimates (5.26) yields

$$
\sum_{s+k=r} \sum_{\substack{s>0 \\ 0 \leq m \leq s-1}}\left\|\Pi\left(\left(\nabla^{m+1} v\right): \nabla^{s-m} D_{t}^{k} h\right)\right\|_{L^{2}(\partial \Omega)} \leq C_{r}\left(K, M, c_{0}, \frac{1}{\epsilon}, E_{r-1}^{*}\right) \sqrt{E_{r}^{*}}
$$

Similarly, (5.59) can be bounded by

- If $m=0$ or $m=r-2$, we have

$$
\begin{array}{r}
\| \Pi\left((\nabla v): \nabla^{r} h\left\|_{L^{2}(\partial \Omega)} \leq\right\| \nabla v\left\|_{L^{\infty}}\right\| \nabla^{r} h \|_{L^{2}(\partial \Omega)},\right. \\
\| \Pi\left(\left(\nabla^{r-1} v\right) \cdot \nabla^{2} h\left\|_{L^{2}(\partial \Omega)} \leq\right\| \nabla^{2} h\left\|_{L^{\infty}}\right\| \nabla^{r-1} v \|_{L^{2}(\partial \Omega)} .\right.
\end{array}
$$

- If $1 \leq m \leq r-3$, we have

$$
\begin{aligned}
\left\|\Pi\left(\left(\nabla^{m+1} v\right) \approx \nabla^{r-m} h\right)\right\|_{L^{2}(\partial \Omega)} & \lesssim K \\
\left\|\nabla^{m+1} v\right\|_{L^{2}(\partial \Omega)}^{1 / 2} & \left\|\nabla^{r-m} h\right\|_{L^{2}(\partial \Omega)}^{1 / 2}\left\|\nabla^{m+1} v\right\|_{H^{1}(\partial \Omega)}^{1 / 2}\left\|\nabla^{r-m} h\right\|_{H^{1}(\partial \Omega)}^{1 / 2} .
\end{aligned}
$$

As for (5.60), we recall (e.g., [20]) that when $r \leq 4$, we have

$$
\sum_{s+k=r}\left\|\Pi\left((\nabla h): \nabla^{s} D_{t}^{k} v\right)\right\|_{L^{2}(\partial \Omega)} \lesssim K, M\langle\langle h\rangle\rangle_{r}+\sum_{j \leq r-1}\left\|\nabla^{j} v\right\|_{L^{2}(\partial \Omega)} .
$$

However, when $r \geq 5$, since

$$
\nabla^{s} D_{t}^{k} v=-\nabla^{s+1} D_{t}^{k-1} h+c_{\alpha \beta \gamma}\left(\partial^{\alpha_{1}} v\right) \cdots\left(\partial^{\alpha_{m}} v\right)\left(\partial^{\beta_{1}} D_{t}^{\gamma_{1}} h\right) \cdots\left(\partial^{\beta_{n}} D_{t}^{\gamma_{n}} h\right)
$$

where

$$
\begin{array}{r}
\alpha_{1}+\cdots+\alpha_{m}+\left(\beta_{1}+\gamma_{1}\right)+\cdots+\left(\beta_{n}+\gamma_{n}\right)=r, \\
1 \leq \alpha_{i} \leq r-1, \quad 1 \leq \beta_{j}+\gamma_{j} \leq r-1 .
\end{array}
$$

Then there must be at most one $\alpha_{i} \geq r-2$ and further if $\alpha_{i}=r-1$, the other term must satisfy the a priori assumption (1.28). Moreover, there are at most three $i$ 's such that $\alpha_{i} \geq r-3$. Hence,

$$
\begin{aligned}
\left\|\left(\partial^{\alpha_{1}} v\right) \cdots\left(\partial^{\alpha_{m}} v\right)\left(\partial^{\beta_{1}} D_{t}^{\gamma_{1}} h\right) \cdots\left(\partial^{\beta_{n}} D_{t}^{\gamma_{n}} h\right)\right\|_{L^{2}(\partial \Omega)} \leq \\
C_{r}\left(K, M, \sum_{k \leq r-2}\left\|\nabla^{k} v\right\|_{L^{2}(\partial \Omega)}, \sum_{k \leq r-1}\langle\langle h\rangle\rangle_{k}\right)\left(\sum_{k \leq r-1}\left\|\nabla^{k} v\right\|_{L^{2}(\partial \Omega)}+\sum_{k \leq r}\langle\langle h\rangle\rangle_{k}\right) .
\end{aligned}
$$

Proposition 5.4. Let $r \geq r_{0}>\frac{n}{2}+\frac{3}{2}$, there is a continuous function $\mathcal{T}_{r}>0$ such that if

$$
0<T \leq \mathcal{T}_{r}\left(c_{0}, K, \mathcal{E}(0), E_{r}^{*}(0)\right),
$$

where

$$
\mathcal{E}(t)=\left|\left(\nabla_{N} h(t, \cdot)\right)^{-1}\right|_{L^{\infty}(\partial \Omega)} .
$$

Then any smooth solution of (1.3) for $0 \leq t \leq T$ satisfies

$$
\begin{array}{r}
E_{r}^{*}(t) \leq 2 E_{r}^{*}(0), \\
\mathcal{E}(t) \leq 2 \mathcal{E}(0), \\
g_{a b}(0, y) Z^{a} Z^{b} \lesssim g_{a b}(t, y) Z^{a} Z^{b} \lesssim g_{a b}(0, y) Z^{a} Z^{b},
\end{array}
$$


there exists a $\eta>0$ such that

$$
\begin{array}{r}
|N(x(t, \bar{y}))-N(x(0, \bar{y}))| \lesssim \eta, \quad \bar{y} \in \partial \Omega, \\
|x(t, y)-x(0, y)| \lesssim \eta, \quad y \in \Omega, \\
\left|\frac{\partial x(t, \bar{y})}{\partial y}-\frac{\partial x(0, \bar{y})}{\partial y}\right| \lesssim \eta, \quad \bar{y} \in \partial \Omega,
\end{array}
$$

hold. To prove Proposition 5.4 we will be using Sobolev lemmas. But then we must make sure that we can control the Sobolev constants. By Lemma A.4 and A.5, the Sobolev constants depend on $K=\frac{1}{l_{0}}$, in fact we are allowed to pick a $K$ depending only on initial conditions, which is proved in [5]. On the other hand, the change of the Sobolev constants in time are controlled by a bound for the time derivative of the metric in Lagrangian coordinate. We also need to control the constant $\frac{1}{\epsilon}$ appears to be in the physical sign condition (1.25).

Lemma 5.5. Assume the conditions in Proposition 5.4 hold. Then there are continuous functions $C_{r_{0}}$ such that

$$
\begin{array}{r}
\|\nabla v\|_{L^{\infty}(\Omega)}+\|\nabla h\|_{L^{\infty}(\Omega)} \leq C_{r_{0}}\left(K, c_{0}, E_{0}, \cdots, E_{r_{0}}\right), \\
\left\|\nabla^{2} h\right\|_{L^{\infty}(\Omega)}+\left\|\nabla D_{t} h\right\|_{L^{\infty}(\Omega)} \leq C_{r_{0}}\left(K, c_{0}, E_{0}, \cdots, E_{r_{0}}\right), \\
\|\nabla \cdot \operatorname{curl} v\|_{L^{\infty}(\Omega)} \leq C_{r_{0}}\left(K, E_{0}, \cdots, E_{r_{0}}\right), \\
\left\|e^{\prime}(h) D_{t} h\right\|_{L^{\infty}(\Omega)}+\left\|e^{\prime}(h) D_{t}^{2} h\right\|_{L^{\infty}(\Omega)} \leq C_{r_{0}}\left(K, c_{0}, E_{0}, \cdots, E_{r_{0}}\right), \\
\|\theta\|_{L^{\infty}(\partial \Omega)} \leq C_{r_{0}}\left(K, c_{0}, \mathcal{E}, E_{0}, \cdots, E_{r_{0}}\right), \\
\left|\frac{d}{d t} \mathcal{E}\right| \leq C_{r_{0}}\left(K, \mathcal{E}, E_{0}, \cdots, E_{r_{0}}\right) .
\end{array}
$$

Proof. By Sobolev lemmas, we have

and

$$
\begin{aligned}
\|\nabla v\|_{L^{\infty}(\Omega)} \lesssim K & \sum_{j \leq 3}\left\|\nabla^{j} v\right\|_{L^{2}(\Omega)}, \\
\|\nabla h\|_{L^{\infty}(\Omega)} & \lesssim K \sum_{j \leq 3}\left\|\nabla^{j} h\right\|_{L^{2}(\Omega)},
\end{aligned}
$$

$$
\left\|\nabla^{2} h\right\|_{L^{\infty}(\Omega)}+\left\|\nabla D_{t} h\right\|_{L^{\infty}(\Omega)} \lesssim K \sum_{j \leq 4}\left\|\nabla^{j} h\right\|_{L^{2}(\Omega)}+\sum_{j \leq 3}\left\|\nabla^{j} D_{t} h\right\|_{L^{2}(\Omega)} .
$$

So, as a consequence of our interior and boundary estimates, (5.79)-(5.80) follows. In addition to these, we have

$$
\|\nabla \cdot \operatorname{curl} v\|_{L^{\infty}(\Omega)} \lesssim K \sum_{j \leq 3}\left\|\nabla^{j} \cdot \operatorname{curl} v\right\|_{L^{2}(\Omega)},
$$

and so (5.81) follows. Now, since the assumptions on $e^{\prime}(h)$ yield

$$
\sum_{j=1,2}\left|\nabla^{j} e^{\prime}(h)\right| \leq C\left(M, c_{0}\right)
$$

thus

$$
\left\|e^{\prime}(h) D_{t} h\right\|_{L^{\infty}(\Omega)}+\left\|e^{\prime}(h) D_{t}^{2} h\right\|_{L^{\infty}(\Omega)} \lesssim_{K, M, c_{0}} \sum_{j=1,2}\left(W_{j}+\left\|\nabla^{j} D_{t} h\right\|_{L^{2}(\Omega)}+\left\|\nabla^{j} D_{t}^{2} h\right\|_{L^{2}(\Omega)}\right),
$$

and so (5.82) follows. On the other hand, since

$$
\left|\nabla^{2} h\right| \geq\left|\Pi \nabla^{2} h\right|=\left|\nabla_{N} h\right||\theta| \geq \mathcal{E}^{-1}|\theta|,
$$

so (5.83) follows from (5.80). Lastly, (5.84) is a consequence of

$$
\frac{d}{d t}\left\|\left(-\nabla_{N} h(t, \cdot)\right)^{-1}\right\|_{L^{\infty}(\partial \Omega)} \lesssim\left\|\left(-\nabla_{N} h(t, \cdot)\right)^{-1}\right\|_{L^{\infty}(\partial \Omega)}^{2}\left\|\nabla_{N} h_{t}(t, \cdot)\right\|_{L^{\infty}(\partial \Omega)},
$$

and (5.80). 


\subsubsection{Proof of Proposition 5.4}

Since when $r \geq r_{0}>\frac{n}{2}+\frac{3}{2}$, we have

$$
\left|\frac{d}{d t} E_{r}\right| \leq C_{r}\left(c_{0}, K, \mathcal{E}, E_{0}, \cdots, E_{r_{0}}\right) E_{r}^{*},
$$

and the RHS is in fact a polynomial of $E_{r}^{*}$ with positive coefficients, we get (5.73) from Lemma 5.5 and Gronwall's lemma if $\mathcal{T}_{r}\left(c_{0}, K, \mathcal{E}_{0}, E_{r}^{*}(0)\right)>0$ is sufficiently small. (5.74) is a direct consequence of (5.84). In addition, we get from (5.73) and Lemma 5.5 that

$$
\begin{aligned}
\|\nabla v\|_{L^{\infty}(\Omega)}+\|\nabla h\|_{L^{\infty}(\Omega)} & \leq C\left(c_{0}, K, \mathcal{E}(0), E_{0}(0), \cdots, E_{r_{0}}(0)\right), \\
\left\|\nabla^{2} h\right\|_{L^{\infty}(\Omega)}+\left\|\nabla D_{t} h\right\|_{L^{\infty}(\Omega)}+\|\theta\|_{L^{\infty}(\partial \Omega)} & \leq C\left(c_{0}, K, \mathcal{E}(0), E_{0}(0), \cdots, E_{r_{0}}(0)\right) .
\end{aligned}
$$

It follows from these that, when $0<T \leq \mathcal{T}_{r}\left(c_{0}, K, \mathcal{E}(0), E_{r}^{*}(0)\right)$ with $\mathcal{T}_{r}$ chosen to be sufficiently small,

$$
\begin{array}{r}
\|\nabla v(t, \cdot)\|_{L^{\infty}(\Omega)}+\|\nabla h(t, \cdot)\|_{L^{\infty}(\Omega)} \lesssim\|\nabla v(0, \cdot)\|_{L^{\infty}(\Omega)}+\|\nabla h(0, \cdot)\|_{L^{\infty}(\Omega)}, \\
\|\nabla \cdot \operatorname{curl} v(t, \cdot)\|_{L^{\infty}(\Omega)} \lesssim\|\nabla \cdot \operatorname{curl} v(0, \cdot)\|_{L^{\infty}(\Omega)},
\end{array}
$$

and

$$
\begin{aligned}
\left\|\nabla^{2} h(t, \cdot)\right\|_{L^{\infty}(\Omega)}+\left\|\nabla D_{t} h(t, \cdot)\right\|_{L^{\infty}(\Omega)} & +\|\theta(t, \cdot)\|_{L^{\infty}(\partial \Omega)} \\
& \lesssim\left\|\nabla^{2} h(0, \cdot)\right\|_{L^{\infty}(\Omega)}+\left\|\nabla D_{t} h(0, \cdot)\right\|_{L^{\infty}(\Omega)}+\|\theta(0, \cdot)\|_{L^{\infty}(\partial \Omega)},
\end{aligned}
$$

where $0<t \leq T$.

On the other hand, we have

$$
\begin{aligned}
\|v(t, \cdot)\|_{L^{\infty}(\Omega)} & \lesssim\|v(0, \cdot)\|_{L^{\infty}(\Omega)}, \\
\|\rho(t, \cdot)\|_{L^{\infty}(\Omega)} & \lesssim\|\rho(0, \cdot)\|_{L^{\infty}(\Omega)} .
\end{aligned}
$$

In fact, (5.90) follows since $D_{t} v=-\partial h-\mathfrak{e}_{n}$ and (5.85), whereas (5.91) follows since $\left|D_{t} \rho\right| \leq|\rho \operatorname{div} v|$. Now, (5.75) follows because $D_{t} g$ behaves like $\nabla v$. Furthermore, (5.76) follows from

$$
D_{t} N_{a}=-\frac{1}{2} N_{a}\left(D_{t} g^{c d}\right) N_{c} N_{d},
$$

and (5.75) . On the other hand, since by the definition of the Lagrangian coordinate, we have

$$
D_{t} x(t, y)=v(t, x(t, y)),
$$

and so (5.77) follows since (5.90). Lastly, because

$$
D_{t} \frac{\partial x}{\partial y}=\frac{\partial v(t, x)}{\partial x} \frac{\partial x}{\partial y},
$$

(5.78) follows since (5.79).

We close this section by briefly going over the idea which shows that one can choose $K$ depends only on the initial conditions.

Lemma 5.6. Let $0 \leq \eta \leq 2$ be a fixed number, define $l_{1}=l_{1}(\eta)$ to be the largest number such that

$$
\left|N\left(\bar{x}_{1}\right)-N\left(\bar{x}_{2}\right)\right| \leq \eta, \quad \text { whenever }\left|\bar{x}_{1}-\bar{x}_{2}\right| \leq l_{1}, \bar{x}_{1}, \bar{x}_{2} \in \partial \mathcal{D}_{t} .
$$

Suppose $|\theta| \leq K$, we recall that $l_{0}$ is the injective radius defined in Section 1.4, then

$$
\begin{gathered}
l_{0} \geq \min \left(l_{1} / 2,1 / K\right), \\
l_{1} \geq \min \left(2 l_{0}, \eta / K\right) .
\end{gathered}
$$


Proof. See Lemma 3.6 of $[5]$

In fact, Theorem 5.6 shows that $l_{0}$ and $l_{1}$ are comparable to each other as long as the free surface is regular.

Lemma 5.7. Fix $\eta>0$ sufficiently small, let $\mathcal{T}$ be in Proposition 5.4. Pick $l_{1}>0$ such that, whenever $\left|x\left(0, y_{1}\right)-x\left(0, y_{2}\right)\right| \leq 2 l_{1}$,

$$
\left|N\left(x\left(0, y_{1}\right)\right)-N\left(x\left(0, y_{2}\right)\right)\right| \leq \frac{\eta}{2} .
$$

Then if $t \leq \mathcal{T}$ we have

$$
\left|N\left(x\left(t, y_{1}\right)\right)-N\left(x\left(t, y_{2}\right)\right)\right| \leq \eta
$$

whenever $\left|x\left(t, y_{1}\right)-x\left(t, y_{2}\right)\right| \leq l_{1}$.

Proof. We have

$$
\begin{aligned}
\mid N\left(x\left(t, y_{1}\right)\right) & -N\left(x\left(t, y_{2}\right)\right) \mid \\
\leq & \left|N\left(x\left(t, y_{1}\right)\right)-N\left(x\left(0, y_{1}\right)\right)\right|+\left|N\left(x\left(0, y_{1}\right)\right)-N\left(x\left(0, y_{2}\right)\right)\right|+\left|N\left(x\left(0, y_{2}\right)\right)-N\left(x\left(t, y_{2}\right)\right)\right|,
\end{aligned}
$$

and so (5.93) follows from (5.76) and (5.77).

Theorem 5.7 allows us to pick

$$
l_{1}(t) \leq \frac{l_{1}(0)}{2},
$$

in other words, we have if $\frac{1}{l_{1}(0)} \leq \frac{K}{2}$, then

$$
\frac{1}{l_{1}(t)} \leq K
$$

Therefore, Theorem 5.6 yields

$$
\frac{1}{l_{0}(t)} \leq K
$$

\section{Incompressible limit}

We consider the Euler equations depending on the sound speed $\kappa$, i.e.,

$$
\left\{\begin{array}{l}
D_{t} v_{\kappa}=-\partial h_{\kappa}-\mathfrak{e}_{n} \\
\operatorname{div} v_{\kappa}=-D_{t} e_{\kappa}(h) .
\end{array}\right.
$$

Here, we recall that the sound speed $\kappa$ is defined by

$$
\kappa:=\left.p_{\kappa}^{\prime}(\rho)\right|_{\rho=1} .
$$

The purpose of this section is to provide an uniform energy estimate that can be carried over to the incompressible water wave and prove the convergence of $v_{\kappa}, h_{\kappa}$ as $\kappa \rightarrow \infty$. In order to do so, we impose the following conditions on $\rho_{\kappa}(h)$ :

$$
\rho_{\kappa}(h) \rightarrow 1, \quad \text { and hence } e_{\kappa}(h) \rightarrow 0, \quad \text { as } \kappa \rightarrow \infty,
$$

and for each fixed $r \geq 1$, there exists a constant $c_{0}$ such that

$$
\left|e_{\kappa}^{(k)}(h)\right| \leq c_{0}, \quad \text { and }\left|e_{\kappa}^{(k)}(h)\right| \leq c_{0}\left|e_{\kappa}^{\prime}(h)\right|^{k} \leq c_{0}\left|e_{\kappa}^{\prime}(h)\right|, \quad \text { if } k \leq r+1 .
$$

Remark. We remark here that the conditions (6.2)-(6.3) are satisfied if the equation of state is of the form $p(\rho)=C_{\gamma} \kappa\left(\rho^{\gamma}-1\right)$. 
Proposition 6.1. Let $\widetilde{E}_{r, \kappa}$ be defined as

$$
\widetilde{E}_{r, \kappa}=\sum_{s+k=r} E_{s, k}+K_{r}+\sum_{1 \leq j \leq r+1} \widetilde{W}_{j}
$$

where $E_{s, k}$ and $K_{r}$ are defined as (1.13)-(1.14), and

$$
\widetilde{W}_{j}=\frac{1}{2}\left\|e_{\kappa}^{\prime}(h) D_{t}^{j} h_{\kappa}\right\|_{L^{2}(\Omega)}+\frac{1}{2}\left\|\sqrt{e_{\kappa}^{\prime}(h)} \nabla D_{t}^{j-1} h_{\kappa}\right\|_{L^{2}(\Omega)} .
$$

For $r \geq r_{0}>\frac{n}{2}+\frac{3}{2}$, there is a continuous function $\mathcal{T}_{r}>0$ such that if $0<T \leq \mathcal{T}_{r}$, then any smooth solution of (1.3) for $0 \leq t \leq T$ satisfies

$$
\widetilde{E}_{r, \kappa}^{*}(t) \leq 2 \widetilde{E}_{r, \kappa}^{*}(0)
$$

provided the physical sign condition $-\left.\nabla_{N} h_{\kappa}\right|_{\partial \Omega} \geq \epsilon>0$ holds.

Based on the analysis we have in Section 5.5. Proposition 6.1 is a direct consequence of the next theorem.

Theorem 6.2. Let $\widetilde{E}_{r, \kappa}$ be defined as (6.4), then there are continuous functions $C_{r}$ such that, for each fixed $r$

$$
\left|\frac{d \widetilde{E}_{r, \kappa}(t)}{d t}\right| \leq C_{r}\left(K, \frac{1}{\epsilon}, M, \mathfrak{h}_{\Omega}, c_{0}, \widetilde{E}_{r-1, \kappa}^{*}\right) \widetilde{E}_{r, \kappa}^{*}(t), \quad t \in[0, T]
$$

holds for all $\kappa\left(\mathfrak{h}_{\Omega}\right.$ is defined as $(6.20)$, provided that the assumptions $(6.2)-(6.3)$ and

$$
\begin{aligned}
\left|\theta_{\kappa}\right|+\frac{1}{l_{0}} \leq K, & \text { on } \partial \Omega, \\
-\nabla_{N} h_{\kappa} \geq \epsilon>0, & \text { on } \partial \Omega, \\
1 \leq \rho_{\kappa} \leq M, & \text { in } \Omega, \\
\left|\nabla \cdot \operatorname{curl} v_{\kappa}\right| \leq M, & \text { in } \Omega, \\
\left|e_{\kappa}^{\prime}(h) D_{t} h_{\kappa}\right|+\left|e_{\kappa}^{\prime}(h) D_{t}^{2} h_{\kappa}\right| \leq M, & \text { in } \Omega, \\
\left|\nabla v_{\kappa}\right|+\left|\nabla h_{\kappa}\right|+\left|\nabla^{2} h_{\kappa}\right|+\left|\nabla D_{t} h_{\kappa}\right| \leq M, & \text { in } \Omega .
\end{aligned}
$$

It is easy to see that under a priori assumptions (6.7)-(6.12), the estimates for $\left\|f_{r}\right\|_{L^{2}(\Omega)}$ and $\left\|g_{r}\right\|_{L^{2}(\Omega)}$ (Theorem 4.3) stay unchanged. To prove Proposition 6.1, the analysis we had in the Section 5 implies that it suffices to prove an analogous version of Theorem 5.3 .

Theorem 6.3. Let

$$
\|h\|_{r, 1}=\sum_{k+s=r, k \leq r-2}\left\|\nabla^{s} D_{t}^{k} h\right\|_{L^{2}(\Omega)}+\left\|\sqrt{e^{\prime}(h)} \nabla D_{t}^{r-1} h\right\|_{L^{2}(\Omega)}+\left\|e^{\prime}(h) D_{t}^{r} h\right\|_{L^{2}(\Omega)} .
$$

Then under the a priori assumptions (6.7)-(6.12), there are continuous functions $C_{r}$ such that,

$$
\|v\|_{r, 0}^{2}+\|h\|_{r, 1}^{2} \leq C_{r}\left(K, M, c_{0}, \mathfrak{h}_{\Omega}, \widetilde{E}_{r-1}^{*}\right) \widetilde{E}_{r}^{*},
$$

where $\mathfrak{h}_{\Omega}$ is defined as (6.20). In addition to that,

$$
\left\|D_{t} h\right\|_{r, 1}^{2}+\langle\langle h\rangle\rangle_{r}^{2} \leq C_{r}\left(K, M, c_{0}, \frac{1}{\epsilon}, \widetilde{E}_{r-1}^{*}\right) \widetilde{E}_{r}^{*}
$$

Proof. (6.13) follows from the arguments in Section [5.2] apart from terms of the form $\left\|\nabla D_{t}^{k} h_{\kappa}\right\|_{L^{2}(\Omega)}, 0 \leq$ $k \leq r-1$. There would be no problem to control $\left\|\nabla D_{t}^{k} h_{\kappa}\right\|_{L^{2}(\Omega)}$ via $\left\|\Delta D_{t}^{k} h_{\kappa}\right\|_{L^{2}(\Omega)}$ if $\Omega$ were bounded. This bound is a consequence of the classical Poincaré's inequality, i.e., $\left\|D_{t}^{k} h_{\kappa}\right\|_{L^{2}(\Omega)} \leq C(\operatorname{vol} \Omega)\left\|\nabla D_{t}^{k} h_{\kappa}\right\|_{L^{2}(\Omega)}$. In the case when $\Omega$ is unbounded, we can still bound $\left\|D_{t}^{k} h_{\kappa}\right\|_{L^{2}(\Omega)}$ via $\left\|\nabla D_{t}^{k} h_{\kappa}\right\|_{L^{2}(\Omega)}$, given $D_{t}^{k} h_{\kappa}$ decays fast enough at infinity. Also, we need the following Poincaré' inequality in unbounded domains: 
Lemma 6.4. Let $\Sigma \in \mathbb{R}^{n}$ be a strip with boundary $\partial \Sigma=\Gamma_{1} \cup \Gamma_{2}$, then there exists a constant $C=C\left(\mathfrak{h}_{\Sigma}\right)$ such that

$$
\|u\|_{L^{2}(\Sigma)} \leq C\left(\mathfrak{h}_{\Sigma}\right)|| \nabla u \|_{L^{2}(\Sigma)}, \quad \text { for each } u \in H^{1}(\Sigma),\left.\quad u\right|_{\Gamma_{1}}=0,
$$

where $\mathfrak{h}_{\Sigma}$ is the "height" of $\Sigma$ in the bounded direction.

Proof. Without loss of generality, we assume $\Gamma_{1} \subset\left\{x_{n}=0\right\}$ and $\Sigma$ is bounded in the $x_{n}$-direction. Since $\left.u\right|_{\Gamma_{1}}=0$, we have

$$
\left|u\left(x^{\prime}, x_{n}\right)\right| \leq \int_{0}^{x_{n}}\left|\partial_{n} u\left(x^{\prime}, \tau\right)\right| d \tau,
$$

and so

$$
\left|u\left(x^{\prime}, x_{n}\right)\right|^{2} \leq \mathfrak{h}_{\Sigma} \int_{0}^{\mathfrak{h}_{\Sigma}}\left|\partial_{n} u\left(x^{\prime}, \tau\right)\right|^{2} d \tau
$$

Hence, (6.15) follows by integrating this with respect to $x=\left(x^{\prime}, x_{n}\right)$ with $C=\mathfrak{h}_{\Sigma}^{2}$.

Now, since $\left.D_{t}^{k} h_{\kappa}\right|_{t=0} \in L_{w}^{2}(\Omega)$ with $w(x)=\left(1+|x|^{2}\right)^{\mu}, \mu \geq 2$ whenever $\kappa$ is sufficient large (Theorem 7.1), there exists strip $\Omega_{\bar{\epsilon}} \subset \Omega$, chosen independent of $\kappa$ and bounded in $x_{n}$-direction, such that

$$
\int_{\Omega-\Omega_{\bar{\epsilon}}}\left|D_{t}^{k} h_{\kappa}\right|^{2} d y \leq \bar{\epsilon}, \quad \text { for each } 0 \leq k \leq r-1,
$$

for all $\kappa$ sufficiently large, and this yields

$$
\left\|D_{t}^{k} h_{\kappa}\right\|_{L^{2}(\Omega)} \lesssim\left\|D_{t}^{k} h_{\kappa}\right\|_{L^{2}\left(\Omega_{\bar{\epsilon}}\right)}
$$

Next, Lemma 6.4 implies

$$
\begin{aligned}
& \left\|D_{t}^{k} h_{\kappa}\right\|_{L^{2}(\Omega)} \leq C\left(\mathfrak{h}_{\Omega}\right)\left\|\nabla D_{t}^{k} h_{\kappa}\right\|_{L^{2}\left(\Omega_{\bar{\epsilon}}\right)} \\
& \quad \leq\left\|\nabla D_{t}^{k} h_{\kappa}\right\|_{L^{2}(\Omega)}, \text { where } \mathfrak{h}_{\Omega} \text { is the height of } \Omega_{\bar{\epsilon}},
\end{aligned}
$$

and hence, we have

$$
\begin{aligned}
& \left\|\nabla D_{t}^{k} h_{\kappa}\right\|_{L^{2}(\Omega)}^{2} \leq\left\|D_{t}^{k} h_{\kappa}\right\|_{L^{2}(\Omega)}\left\|\Delta D_{t}^{k} h_{\kappa}\right\|_{L^{2}(\Omega)} \leq \\
& \quad C\left(\mathfrak{h}_{\Omega}\right)\left\|\nabla D_{t}^{k} h_{\kappa}\right\|_{L^{2}(\Omega)}\left(\left\|e_{\kappa}^{\prime}(h) D_{t}^{k+2} h_{\kappa}\right\|_{L^{2}(\Omega)}+\left\|f_{k+1}\right\|_{L^{2}(\Omega)}+\left\|g_{k+1}\right\|_{L^{2}(\Omega)}\right) .
\end{aligned}
$$

Therefore

$$
\sum_{0 \leq k \leq r-1}\left\|\nabla D_{t}^{k} h_{\kappa}\right\|_{L^{2}(\Omega)} \leq C\left(\mathfrak{h}_{\Omega}\right)\left(\sum_{1 \leq j \leq r+1} \widetilde{W}_{j}+\sum_{0 \leq j \leq r}\left(\left\|f_{j}\right\|_{L^{2}(\Omega)}+\left\|g_{j}\right\|_{L^{2}(\Omega)}\right)\right)
$$

The only thing that we have to check at this point is that the estimates for $\left\|f_{j}\right\|_{L^{2}(\Omega)}$ and $\left\|g_{j}\right\|_{L^{2}(\Omega)}, 2 \leq j \leq r$ does not rely on $\left\|\nabla D_{t}^{j-1} h\right\|_{L^{2}(\Omega)}$, but this is just Theorem 4.3. In addition, (6.14) is just (5.26) and it is proved in Section [5.3. This concludes the proof for Theorem 6.3, and hence Theorem 6.2. Proposition 6.1 then follows from repeating the arguments in Section 5.6 .

\subsection{Passing $\left(v_{\kappa}, h_{\kappa}\right)$ to the limit}

The uniform energy bound $\widetilde{E}_{r, \kappa}^{*}(t) \leq 2 \widetilde{E}_{r, \kappa}^{*}(0)$ allows us to prove that the solution of the compressible Euler equations (6.1) converges as $\kappa \rightarrow \infty$. To be more precise, we prove: 
Theorem 6.5. Let $u_{0}$ be a divergence free vector field such that its corresponding pressure $p_{0}$, defined by $\Delta p_{0}=-\left(\partial_{i} u_{0}^{k}\right)\left(\partial_{k} u_{0}^{i}\right)$ and $\left.p_{0}\right|_{\partial \mathcal{D}_{0}}=0$, satisfies the physical condition $-\left.\nabla_{N} p_{0}\right|_{\partial \mathcal{D}_{0}} \geq \epsilon>0$. Let $(u, p)$ be the solution of the incompressible free boundary Euler equations with data $u_{0}$, i.e.

$$
\rho_{0} D_{t} u=-\partial p, \quad \operatorname{div} u=0,\left.\quad p\right|_{\partial \mathcal{D}_{0}}=0,\left.\quad u\right|_{t=0}=u_{0}
$$

with the constant density $\rho_{0}=1$. Furthermore, let $\left(v_{\kappa}, h_{\kappa}\right)$ be the solution for the compressible Euler equations (6.1), with the density function $\rho_{\kappa}: h \rightarrow \rho_{\kappa}(h)$, and the initial data $v_{0 \kappa}$ and $h_{0 \kappa}$, satisfying the compatibility condition up to order $r+1$, as well as the physical sign condition (6.8). Suppose that $\rho_{\kappa} \rightarrow \rho_{0}=1, v_{0 \kappa} \rightarrow u_{0}$ and $h_{0 \kappa} \rightarrow p_{0}$ as $\kappa \rightarrow \infty$, such that $\widetilde{E}_{r, \kappa}^{*}(0), r \geq 4$ is bounded uniformly independent of $\kappa$, then

$$
\left(v_{\kappa}, h_{\kappa}\right) \rightarrow(u, p) \quad \text { in } C^{r-2}([0, T], \Omega),
$$

where $C^{l}([0, T], \Omega)$ consists all functions $u(t, x)$ with $\nabla^{s} D_{t}^{k} u(t, \cdot), s+k \leq l$ continuous in $\Omega$.

Proof. Theorem 6.3 together with Sobolev lemma would imply

$$
\begin{gathered}
\sum_{s+k=r-2}\left\|\nabla^{s} D_{t}^{k} v_{\kappa}\right\|_{L^{\infty}(\Omega)}+\sum_{s+k=r-2}\left\|\nabla^{s} D_{t}^{k} h_{\kappa}\right\|_{L^{\infty}(\Omega)} \lesssim_{K} \\
\sum_{s+k \leq r}\left\|\nabla^{s} D_{t}^{k} v_{\kappa}\right\|_{L^{2}(\Omega)}+\sum_{s+k \leq r}\left\|\nabla^{s} D_{t}^{k} h_{\kappa}\right\|_{L^{2}(\Omega)} \leq 2 \widetilde{E}_{r, \kappa}^{*}(0) .
\end{gathered}
$$

Furthmore, this implies that when $s+k=r-2$, we have

$$
\nabla^{s} D_{t}^{k} v_{\kappa}, \nabla^{s} D_{t}^{k} h_{\kappa} \in C^{0, \frac{1}{2}}(\Omega)
$$

where $C^{0, \frac{1}{2}}(\Omega)$ is the Hölder space. Now, Arzela-Ascoli theorem shows that the solution $\left(v_{\kappa}, h_{\kappa}\right)$ is uniformly bounded and equicontinuous in $C^{r-2}([0, T] \times \Omega)$. Therefore, $\left(v_{\kappa}, h_{\kappa}\right)$ converges in $C^{r-2}$ with $r \geq 4$, after possibly passing to a subsequence. Finally, $\left(v_{\kappa}, h_{\kappa}\right) \rightarrow(u, p)$ follows from $D_{t}^{2} e_{\kappa}(h) \rightarrow 0$, which is a consequence of $\left\|h_{\kappa}\right\|_{C^{2}([0, T], \Omega)}$ is bounded independent of $\kappa$.

\section{The initial data and the physical condition}

The purpose of this section is to prove that there exist initial data satisfying the compatibility condition in some weighted Sobolev spaces that satisfies the conditions in Theorem 6.5. In [20, we proved that given a smooth initial domain $\mathcal{D}_{0}$ with $\operatorname{vol} \mathcal{D}_{0}<\infty$, and let $u_{0}$ be a divergence free vector field such that its corresponding pressure $p_{0}$, defined by $-\Delta p_{0}=\left(\partial_{i} u_{0}^{k}\right)\left(\partial_{k} u_{0}^{i}\right)$ and $\left.p_{0}\right|_{\partial \mathcal{D}_{0}}=0$, satisfies the physical condition $-\left.\nabla_{N} p_{0}\right|_{\partial \mathcal{D}_{0}} \geq \epsilon>0$. We are able to construct a sequence of initial data $\left(v_{0}, h_{0}\right)=\left(v_{0 \kappa}, h_{0 \kappa}\right)$, satisfying the compatibility condition up to order 5, i.e., $\left.D_{t}^{k} h\right|_{\{0\} \times \partial \mathcal{D}_{0}}=0$ for $0 \leq k \leq 5$, such that $\left(v_{0 \kappa}, h_{0 \kappa}\right) \rightarrow\left(u_{0}, p_{0}\right)$ and $E_{r, \kappa}^{*}(0)$ are uniformly bounded for $r \leq 4$.

In the case of a water wave, we also need to prove that the data $\left(v_{0 \kappa}, h_{0 \kappa}\right)$ admits sufficient decay. This can be achieved by constructing data in weighted Sobolev spaces. Our goal is to prove:

Theorem 7.1. For each fixed $r \geq 4$, given the initial domain $\mathcal{D}_{0}$ is unbounded, diffeomorphic to the half space $\left\{x \in \mathbb{R}^{n}: x_{n} \leq 0\right\}$, and any divergence free $u_{0} \in H_{w}^{s}, s \geq r+1$ (see Definition 7.1), where $w=\left(1+|x|^{2}\right)^{\mu}, \mu \geq 2$, such that its corresponding pressure $p_{0}$ (defined as above) verifies the physical sign condition. Then there exist data $v_{0}=v_{0, \kappa}$ and $h_{0}=h_{0, \kappa}$, satisfying the compatibility condition up to order $r+1$, i.e.,

$$
\left.h_{k}\right|_{\partial \mathcal{D}_{0}}=\left.h_{k, \kappa}\right|_{\partial \mathcal{D}_{0}}=0, \quad 0 \leq k \leq r+1
$$

such that the quantities

$$
\left\|v_{0, \kappa}\right\|_{H_{w}^{s}\left(\mathcal{D}_{0}\right)} \quad \text { and } \quad \sum_{k=0}^{r}\left\|h_{k, \kappa}\right\|_{H_{w}^{s-k}\left(\mathcal{D}_{0}\right)}, \quad s \geq r+1
$$

are uniformly bounded independent of $\kappa$. In addition, we have $v_{0 \kappa} \rightarrow u_{0}$ and $h_{0 \kappa} \rightarrow p_{0}$ in $C^{1}\left(\mathcal{D}_{0}\right)$. 


\subsection{Existence of initial data in weighted Sobolev spaces}

Definition 7.1. (The weighted Sobolev spaces)

Let $\mathbb{D} \subset \mathbb{R}^{n}$ be a domain and $w(x)=\left(1+|x|^{2}\right)^{\mu}, \mu \geq 2$. For $p \in[1, \infty)$, we let $L_{w}^{p}(\mathbb{D})$ be the Banach space consists of functions $u$ such that

$$
\|u\|_{L_{w}^{p}(\mathbb{D})}:=\left(\int_{\mathbb{D}}|u(x)|^{p} w(x) d x\right)^{1 / p}<\infty .
$$

In addition, for any positive integer $s$, we let $W_{w}^{s, p}(\mathbb{D})$ to be the corresponding weighted Sobolev spaces with the norm

$$
\|u\|_{W_{w}^{s, p}(\mathbb{D})}=\sum_{|\alpha| \leq s}\left\|\nabla^{\alpha} u\right\|_{L_{w}^{p}(\mathbb{D})} .
$$

Finally, $H_{w}^{s}(\mathbb{D}):=W_{w}^{s, 2}(\mathbb{D})$ by convention.

We assume $\mathcal{D}_{0}=\Omega$ by a slight abuse of notation. Theorem 7.1 can be achieved by solving:

$$
\left\{\begin{array}{l}
v_{0}=u_{0}+\partial \phi, \quad \text { in } \Omega, \\
\Delta \phi=-e_{\kappa}^{\prime}\left(h_{0}\right) h_{1}, \quad \text { in } \Omega, \text { and }\left.\phi\right|_{\partial \Omega}=0, \\
\Delta h_{k}=e_{\kappa}^{\prime}\left(h_{0}\right) h_{k+2}+F_{k}+G_{k}, \quad \text { in } \Omega, \text { and }, 0 \leq k \leq r-1, \\
h_{r}=h_{r+1}=0, \quad \text { in } \Omega .
\end{array}\right.
$$

Here, $F_{k}:=\left.f_{k+1}\right|_{t=0}$ and $G_{k}=\left.g_{k+1}\right|_{t=0}$, and hence

$$
F_{k}=c_{\alpha \beta}^{\gamma, k}\left(\partial^{\alpha_{1}} v_{0}\right) \cdots\left(\partial^{\alpha_{m}} v_{0}\right)\left(\partial^{\beta_{1}} h_{\gamma_{1}}\right) \cdots\left(\partial^{\beta_{n}} h_{\gamma_{n}}\right),
$$

where

I. $\alpha_{1}+\cdots+\alpha_{m}+\left(\beta_{1}+\gamma_{1}\right)+\cdots+\left(\beta_{n}+\gamma_{n}\right)=k+2$

II. $1 \leq \alpha_{1} \leq \cdots \leq \alpha_{m} \leq k+1$

III. $1 \leq \beta_{1}+\gamma_{1} \leq \cdots \leq \beta_{n}+\gamma_{n} \leq k+1$ and $\beta_{j} \geq 1, \gamma_{j} \leq k-1$ for all $j$

and

$$
G_{k}=c^{\gamma_{1} \cdots \gamma_{m}, k} e_{\kappa}^{(m)}\left(h_{0}\right) h_{\gamma_{1}} \cdots h_{\gamma_{m}},
$$

where

I. $\gamma_{1}+\cdots+\gamma_{m}=k+2$

II. $1 \leq \gamma_{1} \leq \cdots \leq \gamma_{m} \leq k+1$

We show the existence of solution for (17.1) via successive approximation starting from the solution $\left(h_{0}^{0}, h_{1}^{0}, \cdots, h_{r-1}^{0}\right)$ that solves

$$
\Delta h_{k}^{0}=F_{k}\left(\partial^{\alpha} u_{0}, \partial^{\beta_{0}} h_{0}^{0}, \cdots, \partial^{\beta_{k-1}} h_{k-1}^{0}\right), \quad 0 \leq k \leq r-1
$$

and we define $\left(h_{0}^{\nu}, \cdots, h_{r-1}^{\nu}\right)$ inductively by solving

$$
\left\{\begin{array}{l}
v_{0}^{\nu}=u_{0}+\partial \phi^{\nu} \\
\Delta \phi^{\nu}=-e_{\kappa}^{\prime}\left(h_{0}^{\nu-1}\right) h_{1}^{\nu-1} \\
\Delta h_{k}^{\nu}=e_{\kappa}^{\prime}\left(h_{0}^{\nu-1}\right) h_{k+2}^{\nu-1}+F_{k}^{\nu}+G_{k}^{\nu-1}, \quad 0 \leq k \leq r-3 \\
\Delta h_{k}^{\nu}=F_{k}^{\nu}+G_{k}^{\nu-1}, \quad k=r-2, r-1 \\
\left.\phi^{\nu}\right|_{\partial \Omega}=\left.h_{k}^{\nu}\right|_{\partial \Omega}=0 .
\end{array}\right.
$$

\footnotetext{
${ }^{7}$ The system (7.1) is generated by re-writing the wave equations (4.7) as Laplace equations. We refer 20] for the detailed construction. Also, in [20], we only considered the case when $e_{\kappa}(h)=\kappa^{-1} h$, and we shall solve the general case here as well.
} 
Here,

$$
\begin{array}{r}
F_{k}^{\nu}=F_{k}\left(\partial^{\alpha} v_{0}^{\nu}, \partial^{\beta_{0}} h_{0}^{\nu}, \cdots, \partial^{\beta_{k-1}} h_{k-1}^{\nu}\right), \\
G_{k}^{\nu-1}=G_{k}\left(h_{0}^{\nu-1}, \cdots, h_{k+1}^{\nu-1}\right) .
\end{array}
$$

Now, we define that for $0 \leq k \leq r-1$,

$$
\begin{aligned}
m_{k}^{\nu} & :=\left\|h_{k}^{\nu}\right\|_{H_{w}^{s-k}(\Omega)}, \quad s \geq r+1 \\
m_{*}^{\nu} & :=\sum_{k \leq r-1} m_{k}^{\nu}+\left\|v_{0}^{\nu}\right\|_{H_{w}^{s}(\Omega)} .
\end{aligned}
$$

According to the elliptic estimate (Theorem A.10), we have

$$
\begin{array}{r}
\left\|\nabla \phi^{\nu}\right\|_{H_{w}^{s}(\Omega)} \leq C\left\|e_{\kappa}^{\prime}\left(h_{0}^{\nu-1}\right) h_{1}^{\nu-1}\right\|_{H_{w}^{s-1}(\Omega)}, \\
\left\|h_{k}^{\nu}\right\|_{H_{w}^{s-k}(\Omega)} \leq C\left(\left\|e_{\kappa}^{\prime}\left(h_{0}^{\nu-1}\right) h_{k+2}^{\nu-1}\right\|_{H_{w}^{s-k-2}(\Omega)}+\left\|F_{k}^{\nu}\right\|_{H_{w}^{s-k-2}(\Omega)}+\left\|G_{k}^{\nu-1}\right\|_{H_{w}^{s-k-2}(\Omega)}\right), \quad 0 \leq k \leq r-3 . \\
\left\|h_{k}^{\nu}\right\|_{H_{w}^{s-k}(\Omega)} \leq C\left(\left\|F_{k}^{\nu}\right\|_{H_{w}^{s-k-2}(\Omega)}+\left\|G_{k}^{\nu-1}\right\|_{H_{w}^{s-k-2}(\Omega)}\right), \quad k=r-2, r-1 .
\end{array}
$$

On the other hand, the Sobolev inequalities are still valid for weighted Sobolev spaces; in other words, we have

Lemma 7.2. (Weighted Sobolev inequalities) Let $w(x)=\left(1+|x|^{2}\right)^{\mu}$, and let $\Omega$ be a domain with $C^{1}$ boundary, then

(a) $\|u\|_{L_{w}^{n p /(n-s p)}(\Omega)} \leq C\|u\|_{W_{w}^{s, p}(\Omega)}$, if $s p<n$.

(b) $\|u\|_{L^{\infty}(\Omega)} \leq C\|u\|_{W_{w}^{s, p}(\Omega)}$, if $s p>n$.

Proof. Part (a) follows from the proof given by Evans [11] with a slight modification. Part (b) is a direct consequence of the standard Sobolev inequality.

Remark. The above lemma can be generalized to a much larger class of weighted Sobolev spaces. We refer Turesson 25] Chapter 3 for the details.

Lemma 7.2 allows us to get the bounds for $\left\|F_{k}^{\nu}\right\|_{H_{w}^{s-k-2}}$ and $\left\|G_{k}^{\nu-1}\right\|_{H_{w}^{s-k-2}}$.

\subsubsection{Bounds for $\left\|F_{k}^{\nu}\right\|_{H_{w}^{s-k-2}}$}

Since $F_{k}^{\nu}$ is a sum of products of the form (7.2), we have

- If the product involves less than 4 terms, i.e., $m+n \leq 3$, then

$$
\begin{aligned}
& \left\|\left(\partial^{\alpha_{1}} v_{0}^{\nu}\right) \cdots\left(\partial^{\alpha_{m}} v_{0}^{\nu}\right)\left(\partial^{\beta_{1}} h_{\gamma_{1}}^{\nu}\right) \cdots\left(\partial^{\beta_{n}} h_{\gamma_{n}}^{\nu}\right)\right\|_{H_{w}^{s-k-2}} \\
& \quad \leq C\left\|\partial^{\alpha_{1}} v_{0}^{\nu}\right\|_{H_{w}^{s-k-1}} \cdots\left\|\partial^{\alpha_{m}} v_{0}^{\nu}\right\|_{H_{w}^{s-k-1}}\left\|\partial^{\beta_{1}} h_{\gamma_{1}}^{\nu}\right\|_{H_{w}^{s-k-1}} \cdots\left\|\partial^{\beta_{n}} h_{\gamma_{n}}^{\nu}\right\|_{H_{w}^{s-k-1}} \\
& \quad \leq p\left(\left\|v_{0}^{\nu}\right\|_{H_{w}^{s}}, m_{0}^{\nu}, \cdots, m_{k-1}^{\nu}\right)
\end{aligned}
$$

for some polynomial $p$, where the last inequality is because $\beta_{j} \leq k+1-\gamma_{j}$ and $\gamma_{j} \leq k-1$.

- If the product involves at least 4 terms, i.e., $m+n \geq 4$. Then we must have $1 \leq \alpha_{i} \leq \alpha_{m} \leq k-1$ and $1 \leq \beta_{j}+\gamma_{j} \leq \beta_{n}+\gamma_{n} \leq k-1$. But since $\beta_{j} \geq 1$, we have

$$
\begin{aligned}
& \left\|\left(\partial^{\alpha_{1}} v_{0}^{\nu}\right) \cdots\left(\partial^{\alpha_{m}} v_{0}^{\nu}\right)\left(\partial^{\beta_{1}} h_{\gamma_{1}}^{\nu}\right) \cdots\left(\partial^{\beta_{n}} h_{\gamma_{n}}^{\nu}\right)\right\|_{H_{w}^{s-k-2}} \\
& \quad \leq C\left\|\partial^{\alpha_{1}} v_{0}^{\nu}\right\|_{H_{w}^{s-k}} \cdots\left\|\partial^{\alpha_{m}} v_{0}^{\nu}\right\|_{H_{w}^{s-k}}\left\|\partial^{\beta_{1}} h_{\gamma_{1}}^{\nu}\right\|_{H_{w}^{s-k}} \cdots\left\|\partial^{\beta_{n}} h_{\gamma_{n}}^{\nu}\right\|_{H_{w}^{s-k}} \\
& \quad \leq p\left(\left\|v_{0}^{\nu}\right\|_{H_{w}^{s}}, m_{0}^{\nu}, \cdots, m_{k-2}^{\nu}\right) .
\end{aligned}
$$


7.1.2 Bounds for $\left\|G_{k}^{\nu-1}\right\|_{H_{w}^{s-k-2}}$ and $\left\|e_{\kappa}^{\prime}\left(h_{0}^{\nu-1}\right) h_{k+2}^{\nu-1}\right\|_{H_{w}^{s-k-2}}$

Since $\left|e^{(m)}\left(h_{0}\right)\right| \leq c\left|e_{\kappa}^{\prime}\left(h_{0}\right)\right|^{m}$, (7.3) together with the weighted Sobolev inequalities imply

$$
\left\|G_{k}^{\nu-1}\right\|_{H_{w}^{s-k-2}} \leq q\left(e_{\kappa}^{\prime}\left(h_{0}^{\nu-1}\right) m_{0}^{\nu-1}, \cdots, e_{\kappa}^{\prime}\left(h_{0}^{\nu-1}\right) m_{k+1}^{\nu-1}\right), \quad 0 \leq k \leq r-2
$$

and since $h_{r}=0$,

$$
\left\|G_{r-1}^{\nu-1}\right\|_{H_{w}^{s-r-1}} \leq q\left(e_{\kappa}^{\prime}\left(h_{0}^{\nu-1}\right) m_{0}^{\nu-1}, \cdots, e_{\kappa}^{\prime}\left(h_{0}^{\nu-1}\right) m_{r-1}^{\nu-1}\right),
$$

for some polynomial $q$. On the other hand, we have

$$
\|\left. e_{\kappa}^{\prime}\left(h_{0}^{\nu-1}\right) h_{k+2}^{\nu-1}\right|_{H_{w}^{s-k-2}} \leq \widetilde{q}\left(e_{\kappa}^{\prime}\left(h_{0}^{\nu-1}\right) m_{0}^{\nu-1}, e_{\kappa}^{\prime}\left(h_{0}^{\nu-1}\right) m_{k+2}^{\nu-1}\right), \quad 0 \leq k \leq r-3
$$

for some polynomial $\widetilde{q}$.

\subsubsection{Bound for $\left\|v_{0}^{\nu}\right\|_{H_{w}^{s}}$}

The first equation of (7.5) yields

$$
\left\|v_{0}^{\nu}\right\|_{H_{w}^{s}} \leq\left\|u_{0}\right\|_{H_{w}^{s}}+\left\|\nabla \phi^{\nu}\right\|_{H_{w}^{s}}
$$

But since

$$
\left\|e_{\kappa}^{\prime}\left(h_{0}^{\nu-1}\right) h_{1}^{\nu-1}\right\|_{H_{w}^{s-1}} \leq q\left(e_{\kappa}^{\prime}\left(h_{0}^{\nu-1}\right) m_{0}^{\nu-1}, e_{\kappa}^{\prime}\left(h_{0}^{\nu-1}\right) m_{1}^{\nu-1}\right)
$$

and so (7.6) implies

$$
\left\|v_{0}^{\nu}\right\|_{H_{w}^{s}} \leq\left\|u_{0}\right\|_{H_{w}^{s}}+q\left(e_{\kappa}^{\prime}\left(h_{0}^{\nu-1}\right) m_{0}^{\nu-1}, e_{\kappa}^{\prime}\left(h_{0}^{\nu-1}\right) m_{1}^{\nu-1}\right)
$$

\subsubsection{A priori bounds for the full system (7.5)}

We conclude from (7.9)-(17.13) that

$$
m_{k}^{\nu} \leq C e^{\prime} m_{k+2}^{\nu-1}+P\left(\left\|v_{0}^{\nu}\right\|_{H_{w}^{s}}, m_{0}^{\nu}, \cdots, m_{k-1}^{\nu}, e^{\prime} m_{0}^{\nu-1}, \cdots, e^{\prime} m_{k+1}^{\nu-1}\right),
$$

for $0 \leq k \leq r-3$ and

$$
\begin{aligned}
& m_{r-2}^{\nu} \leq P\left(\left\|v_{0}^{\nu}\right\|_{H_{w}^{s}}, m_{0}^{\nu}, \cdots, m_{r-3}^{\nu}, e^{\prime} m_{0}^{\nu-1}, \cdots, e^{\prime} m_{r-1}^{\nu-1}\right) \\
& m_{r-1}^{\nu} \leq P\left(\left\|v_{0}^{\nu}\right\|_{H_{w}^{s}}, m_{0}^{\nu}, \cdots, m_{r-2}^{\nu}, e^{\prime} m_{0}^{\nu-1}, \cdots, e^{\prime} m_{r-1}^{\nu-1}\right)
\end{aligned}
$$

Summing these up, we get

$$
m_{*}^{\nu} \leq P\left(e_{\kappa}^{\prime} m_{*}^{\nu-1},\left\|v_{0}^{\nu}\right\|_{H_{w}^{s}}\right) \leq Q\left(e_{\kappa}^{\prime} m_{*}^{\nu-1},\left\|u_{0}\right\|_{H_{w}^{s}}\right)
$$

for some polynomials $P$ and $Q$ via (7.16). In particular, this implies that $m_{*}^{\nu}$ is uniformly bounded for all $\nu$ by induction whenever $e_{\kappa}^{\prime}$ (and hence $\kappa^{-1}$ ) is sufficiently small. Finally, the existence follows from subtracting two successive systems of (7.4)-(7.5) and the a priori bound (7.20), which is identical to what is in [20].

On the other hand, since $s \geq r+1 \geq 5$ and $\Omega \in \mathbb{R}^{n}, n=2,3$, we have

$$
\left\|v_{0, \kappa}-u_{0}\right\|_{C^{1}(\Omega)} \leq C|| v_{0, \kappa}-u_{0}\left\|_{H_{w}^{s}(\Omega)} \leq\right\| \nabla \phi_{\kappa} \|_{H_{w}^{s}(\Omega)} \leq q\left(e_{\kappa}^{\prime}\left\|h_{0, \kappa}\right\|_{H_{w}^{s}(\Omega)}, e_{\kappa}^{\prime}\left\|h_{1, \kappa}\right\|_{H_{w}^{s-1}(\Omega)}\right),
$$

where $q(0)=0$. This implies $v_{0, \kappa} \rightarrow u_{0}$ in $C^{1}(\Omega)$ since $\left\|h_{k, \kappa}\right\|_{H_{w}^{s-k}}$ are uniformly bounded independent of $\kappa$ and $e_{\kappa}^{\prime} \rightarrow 0$. Similarly, we have

$$
\left\|h_{0, \kappa}-p_{0}\right\|_{H_{w}^{s}} \leq C\left(\left\|e_{\kappa}^{\prime} h_{2, \kappa}\right\|_{H_{w}^{s-2}}+\left\|e_{\kappa}^{\prime \prime} h_{1, \kappa}^{2}\right\|_{H_{w}^{s-2}}+\left(\left\|u_{0}\right\|_{H_{w}^{s}}+\left\|\phi_{\kappa}\right\|_{H_{w}^{s}}\right)\left\|\phi_{\kappa}\right\|_{H_{w}^{s}}\right) .
$$

The RHS $\rightarrow 0$ as $\kappa \rightarrow \infty$ via (7.6) and (7.11). This concludes the proof of Theorem 7.1 
Corollary 7.3. $\widetilde{E}_{r, \kappa}^{*}(0)$ in Proposition 6.1 is uniformly bounded whenever $\kappa^{-1}$ is sufficiently small.

Proof. Since $w(x) \geq 1$, we have

$$
\sum_{k+s \leq r} \int_{\Omega} \rho_{0} Q\left(\partial^{s} h_{k, \kappa}, \partial^{s} h_{k, \kappa}\right) d x \lesssim \sum_{k \leq r}\left\|h_{k, \kappa}\right\|_{H^{r-k}(\Omega)}^{2} \leq \sum_{k \leq r}\left\|h_{k, \kappa}\right\|_{H_{w}^{r-k}(\Omega)}^{2},
$$

and by the trace lemma,

$$
\sum_{k+s \leq r} \int_{\partial \Omega} \rho_{0} Q\left(\partial^{s} h_{k, \kappa}, \partial^{s} h_{k, \kappa}\right) d S \lesssim \sum_{k \leq r}\left\|h_{k, \kappa}\right\|_{H^{r-k}(\partial \Omega)}^{2} \lesssim \sum_{k \leq r}\left\|h_{k, \kappa}\right\|_{H_{w}^{r+1-k}(\Omega)}^{2} .
$$

In addition to these, we have for $r \geq 2$ that

$$
\sum_{k \leq r}\left\|\left.\left(\partial^{r-k} D_{t}^{k} v_{\kappa}\right)\right|_{t=0}||_{L^{2}} \lesssim\right\| v_{0, \kappa} \|_{H_{w}^{r}}+P\left(\left\|v_{0, \kappa}\right\|_{H_{w}^{r-1}}, \sum_{k \leq r-1}\left\|h_{k, \kappa}\right\|_{H_{w}^{r-1-k}}\right),
$$

for some polynomial $P$ via (4.19). This shows when $s+k \geq 2$,

$$
\int_{\Omega} \rho_{0} Q\left(\left.\partial^{s} D_{t}^{k} v_{\kappa}\right|_{t=0},\left.\partial^{s} D_{t}^{k} v_{\kappa}\right|_{t=0}\right) d x
$$

is uniformly bounded. Finally, since $h_{r}=h_{r+1}=0$ in $\Omega$, we have

$$
\sum_{k=1}^{r+1} \widetilde{W}_{k}(0)
$$

is uniformly bounded as well.

\subsection{The physical condition}

It is plausible to assume the physical condition (1.7) on the initial data. We are able to show that for a slight compressible (i.e., $\kappa^{-1}$ is small), irrotational water wave, the quantity $-\nabla_{N} h_{0}$ is pointwisely greater than a positive constant depending only on the geometry of the free surface, as long as the free surface is not self-intersecting. This can be shown via the maximum principle since $h_{0}$ is superharmonic in the case of a slightly compressible and irrotational water wave. The original version of our proof is given by $\mathrm{Wu}[27$. that

In particular, Theorem 7.1 together with Lemma 7.2 yield that for $r=4$, there exists a constant $C$ such

$$
\sum_{k=1,2}\left\|h_{k}\right\|_{L^{\infty}\left(\mathcal{D}_{0}\right)} \leq \frac{C}{\left(1+|x|^{2}\right)^{\mu}}, \quad \mu \geq 2
$$

whenever $\kappa^{-1}$ is sufficiently small. In addition, $\operatorname{since} \operatorname{curl} v=0$, we have $\partial_{i} v_{j}=\partial_{j} v_{i}$ for each $i, j$, and so $h_{0}$ and $x_{n}$ satisfies

$$
-\Delta\left(h_{0}+x_{n}\right)=\left|\partial v_{0}\right|^{2}-\left(e_{\kappa}^{\prime}\left(h_{0}\right) h_{2}+e_{\kappa}^{\prime \prime}\left(h_{0}\right) h_{1}^{2}\right) .
$$

Now, (7.27) guarantees that the right hand side of (7.28) is positive pointwisely whenever $\kappa$ is large (and so $e_{\kappa}^{\prime}$ and $e_{\kappa}^{\prime \prime}$ are small); in other words, $h_{0}+x_{n}$ is superharmonic in the case of a slightly compressible, irrotational liquid. For any $\psi \in C_{c}^{1}\left(\partial \mathcal{D}_{0}\right), \psi \geq 0$, let $\phi$ be the harmonic extension of $\psi$ in $\mathcal{D}_{0}$, i.e., $\phi$ solves

$$
\left\{\begin{array}{l}
\Delta \phi=0, \quad \text { in } \mathcal{D}_{0} \\
\left.\phi\right|_{\partial \mathcal{D}_{0}}=\psi
\end{array}\right.
$$

In fact, it is easy to see that

$$
\phi(x)=o\left(|x|^{2-n}\right), \quad \nabla \phi=o\left(|x|^{1-n}\right),
$$


as $|x| \rightarrow \infty$.

Now, applying the Green's identity $\nabla_{\mathrm{to}} \phi$ and $h_{0}+x_{n}$, we get

$$
\left.\int_{\partial \mathcal{D}_{0}}\left(h_{0}+x_{n}\right) \nabla_{N} \phi-\phi \nabla_{N}\left(h_{0}+x_{n}\right) d S=\int_{\mathcal{D}_{0}} \phi\left(\left|\nabla v_{0}\right|^{2}-e_{\kappa}^{\prime}\left(h_{0}\right) h_{2}-e_{\kappa}^{\prime \prime}\left(h_{0}\right) h_{1}^{2}\right)\right) d x .
$$

But since $h_{0}=0$ on $\partial \mathcal{D}_{0}$, we have

$$
\left.\int_{\partial \mathcal{D}_{0}}-\phi \nabla_{N} h_{0} d S=\int_{\partial \mathcal{D}_{0}}\left(\phi \nabla_{N} x_{n}-x_{n} \nabla_{N} \phi\right) d S+\int_{\mathcal{D}_{0}} \phi\left(\left|\nabla v_{0}\right|^{2}-e_{\kappa}^{\prime}\left(h_{0}\right) h_{2}-e_{\kappa}^{\prime \prime}\left(h_{0}\right) h_{1}^{2}\right)\right) d x .
$$

On the other hand, applying the Green's identity again to $\phi$ and $x_{n}$ on the strip region between $\partial \mathcal{D}_{0}$ and $\left\{x \in \mathbb{R}^{n}: x_{n}=b\right\}$ (with the upward unit normal $N_{b}=e_{n}$ ), we get

$$
\begin{aligned}
\int_{\partial \mathcal{D}_{0}}\left(\phi \nabla_{N} x_{n}-x_{n} \nabla_{N} \phi\right) d S & =\int_{x_{n}=b}\left(\phi \nabla_{N_{b}} x_{n}-x_{n} \nabla_{N_{b}} \phi\right) d S \\
& =\int_{x_{n}=b} \phi d S-b \int_{x_{n}=b} \nabla_{N_{b}} \phi d S \\
& =\int_{x_{n}=b} \phi d S .
\end{aligned}
$$

The integral $\int_{x_{n}=b} \nabla_{N_{b}} \phi d S=0$ is a direct consequence of (7.30) and the Gauss-Green's formula when $n \geq 3$. Therefore,

$$
\begin{aligned}
\int_{\partial \mathcal{D}_{0}}-\phi \nabla_{N} h_{0} d S & \left.=\int_{x_{n}=b} \phi d S+\int_{\mathcal{D}_{0}} \phi\left(\left|\nabla v_{0}\right|^{2}-e_{\kappa}^{\prime}\left(h_{0}\right) h_{2}-e_{\kappa}^{\prime \prime}\left(h_{0}\right) h_{1}^{2}\right)\right) d x \\
& \geq \int_{x_{n}=b} \phi d S .
\end{aligned}
$$

Let $G=G(x, y)$ be the Green's function for the region $\mathcal{D}_{0}$, then by Green's representation formula we have

$$
\phi(x)=\int_{\partial \mathcal{D}_{0}} \psi(y) \nabla_{N} G(x, y) d S(y), \quad \text { for } x \in \mathcal{D}_{0} .
$$

But this then implies

$$
\begin{aligned}
\int_{\partial \mathcal{D}_{0}}-\psi(y) \nabla_{N} h_{0}(y) d S(y) & \geq \int_{x_{n}=b} \phi(x) d S(x) \\
& =\int_{\partial \mathcal{D}_{0}} \psi(y) \int_{x_{n}=b} \nabla_{N} G(x, y) d S(x) d S(y) .
\end{aligned}
$$

Since $\psi \in C_{c}^{1}\left(\partial \mathcal{D}_{0}\right), \psi \geq 0$ is arbitrary, we must have that for each $y \in \partial \mathcal{D}_{t}$,

$$
-\nabla_{N} h_{0}(y) \geq \int_{x_{n}=b} \nabla_{N} G(x, y) d S(x) .
$$

From the maximum principle, we know that there exists $\epsilon>0$ such that $\int_{x_{n}=b} \nabla_{N} G(x, y) d S(x) \geq \epsilon$, for every $y \in \partial \mathcal{D}_{0}$.

Therefore, the following theorem is justified for a slightly compressible, irrotational liquid.

Theorem 7.4. Assume that at time 0 , the water region $\mathcal{D}_{0} \subset \mathbb{R}^{n}, n \geq 3$ is unbounded, diffeomorphic to $\left\{x \in \mathbb{R}^{n}: x_{n} \leq 0\right\}$, whose boundary $\partial \mathcal{D}_{0}$ satisfies $|\theta|+\left|1 / l_{0}\right| \leq K$. Then there exists a positive constant $\epsilon$, depending only on $\partial \mathcal{D}_{0}$ such that

$$
-\nabla_{N} h_{0}(y) \geq \epsilon>0
$$

holds for each $y \in \partial \mathcal{D}_{0}$.

\footnotetext{
${ }^{8}$ Green's identity holds here on unbounded domains because of the decay properties and the $L^{2}$ integrability of our functions involved.
} 
Remark. In the original proof given by $\mathrm{Wu}\left[27\right.$, the pressure $p_{0}$ is automatically superharmonic, since $v_{0}$ is divergence free implies

$$
-\Delta p_{0}=\left|\nabla v_{0}\right|^{2}>0 \text {. }
$$

But we need to put extra effort to make sure that $h_{0}$ is superharmonic in the case of a slightly compressible liquid.

Remark. The presence of the gravity is essential for proving that $-\nabla_{N} h_{0}$ is bounded uniformly below by a positive constant. Since otherwise the term $\int_{\partial \mathcal{D}_{0}}\left(\phi \nabla_{N} x_{n}-x_{n} \nabla_{N} \phi\right) d S$ on the right of (7.32) would be 0 . In this case we can only conclude $-\nabla_{N} h_{0} \geq 0$.

\section{The weighted a priori estimates for the Euler equations}

The purpose of this section is to generalize Proposition 5.1 to weighted $L^{2}$ Sobolev spaces. In the previous section, we have shown that for each fixed $r$, there exist data in $H_{w}^{r+1}$ that satisfying the compatibility condition, and we shall prove that the corresponding weighted energies for the compressible Euler equations remain bounded within short time. This will follow from the analysis we have in Section 5 given the estimates in Section 3 remain valid in weighed Sobolev spaces; in other words, we need to establish the ChristodoulouLindblad type elliptic estimates (Proposition [3.2), as well as the tensor estimate (Proposition 3.3) in the case of weighted spaces. Throughout this section, the weight function $w(x)=\left(1+|x|^{2}\right)^{\mu}, \mu \geq 2$.

\subsection{The weighted Christodoulou-Lindblad type elliptic estimates}

We adopt the notations used in Section 3. Let $\Omega$ be a general domain in $\mathbb{R}^{n}$ and let $\nabla$ be the covariant differentiation with respect to the metric $g_{i j}$ in $\Omega$, and $\bar{\nabla}$ will refer to the covariant differentiation on $\partial \Omega$ with respect to the induced metric $\gamma_{i j}=g_{i j}-N_{i} N_{j}$. We will also assume that the normal $N$ to $\partial \Omega$ is extended to a vector field of $\Omega$ via the geometric normal coordinate satisfying $g_{i j} N^{i} N^{j} \leq 1$ (e.g., Lemma A.1).

Lemma 8.1. Let $u: \Omega \rightarrow \mathbb{R}^{n}$ be a vector field and let $\beta_{k}=\nabla_{i_{1}} \cdots \nabla_{i_{r}} u_{k}:=\nabla_{I}^{r} u_{k}$. If $|\theta|+\frac{1}{l_{0}} \leq K$, then

$$
\int_{\Omega}|\nabla \beta|^{2} w d \mu_{g} \leq C(K) \int_{\Omega}\left(N^{i} N^{j} g^{k l} \gamma^{I J} \nabla_{k} \beta_{I i} \nabla_{l} \beta_{J j}+|\operatorname{div} \beta|^{2}+|\operatorname{curl} \beta|^{2}+|\beta|^{2}\right) w d \mu_{g} .
$$

Here, $\gamma^{I J}=\gamma^{i_{1} j_{1}} \cdots \gamma^{i_{r} j_{r}}$.

Proof. We follow the proof given in Christodoulou-Lindblad [5]. Since $g^{i j}=\gamma^{i j}+N^{i} N^{j}$, we have

$$
|\nabla \beta|^{2}=g^{I J} g^{k l} \nabla_{k} \beta_{I} \nabla_{l} \beta_{J}
$$

can be written as a sum of terms of the form (that is, the normal-tangential form)

$$
N^{i_{1}} N^{j_{1}} \cdots N^{i_{s}} N^{j_{s}} \gamma^{i_{s+1} j_{s+1}} \cdots \gamma^{i_{r} j_{r}} g^{k l} \nabla_{k} \beta_{I} \nabla_{l} \beta_{J},
$$

and if we control the right hand side of (8.1), then we have the bounds for integral of (8.1) for $s=1,2$. However, the following Hodge-type decomposition holds (e.g., [5]): let $q^{I J}$ be any product of factors $q^{i j}$ of the form $g^{i j}, \gamma^{i j}$ or $N^{i} N^{j}$, then

$$
\begin{aligned}
g^{i j} g^{k l} q^{I J} \nabla_{i} \beta_{I k} \nabla_{j} \beta_{J l} \leq & \left(2\left(N^{i} N^{j} g^{k l}+g^{i j} N^{k} N^{l}\right)+2 g^{i k} g^{j l}\right. \\
& \left.+\left(\gamma^{i j} \gamma^{k l}-\gamma^{i k} \gamma^{j l}\right)\right) q^{I J} \nabla_{i} \beta_{I k} \nabla_{j} \beta_{J l} .
\end{aligned}
$$

In addition to this, if $R^{i j k l I J}:=\left(\gamma^{i j} \gamma^{k l}-\gamma^{i k} \gamma^{j l}\right) q^{I J}$, then

$$
\int_{\Omega} R^{i j k l I J} \nabla_{k} \alpha_{I i} \nabla_{j} \beta_{J l} w d \mu_{g}=-\int_{\Omega}\left(\nabla_{k} R^{i j k l I J}\right) \alpha_{I i} \nabla_{j} \beta_{J l} w d \mu_{g}-\int_{\Omega}\left(R^{i j k l I J}\right) \alpha_{I i} \nabla_{j} \beta_{J l}\left(\nabla_{k} w\right) d \mu_{g},
$$

via integrating by parts, since $\left(\gamma^{i j} \gamma^{k l}-\gamma^{i k} \gamma^{j l}\right) \nabla_{j} \nabla_{k} \beta=0$ and $N_{k}\left(\gamma^{i j} \gamma^{k l}-\gamma^{i k} \gamma^{j l}\right)=0$. 
Now, by (8.3) and (8.4), and since the weight satisfies $|\nabla w| \leq \frac{C w}{1+|x|}$, the bounds for integral of (8.1) for $s=1,2$ gives us the integral of (8.1) also for $s=0$. This is because

$$
\left|\left(\gamma^{i j} \gamma^{k l}-\gamma^{i k} \gamma^{j l}\right) q^{I J}\left(\nabla_{i} \beta_{I k} \nabla_{j} \beta_{J l}-\nabla_{k} \beta_{I i} \nabla_{j} \beta_{J l}\right)\right| \leq C|\operatorname{curl} \beta| \cdot|\nabla \beta|,
$$

and

$$
\left|g^{i k} g^{j l} q^{I J} \nabla_{i} \beta_{I k} \nabla_{j} \beta_{J l}\right| \leq C|\operatorname{div} \beta|^{2} .
$$

But then we can use (3.2) to get (8.1).

Lemma 8.2. Let $\beta$ be defined as in the previous lemma. If $|\theta|+\frac{1}{l_{0}} \leq K$, then

$$
\begin{aligned}
& \|\beta\|_{L_{w}^{2}(\partial \Omega)}^{2} \leq C(K)\left(\|\nabla \beta\|_{L_{w}^{2}(\Omega)}^{2}+\|\beta\|_{L_{w}^{2}(\Omega)}^{2}\right), \\
& \|\beta\|_{L_{w}^{2}(\partial \Omega)}^{2} \leq C|| \Pi \beta \|_{L_{w}^{2}(\partial \Omega)}^{2}+C(K)\left(\|\operatorname{div} \beta\|_{L_{w}^{2}(\Omega)}^{2}+\|\operatorname{curl} \beta\|_{L_{w}^{2}(\Omega)}^{2}+\|\beta\|_{L_{w}^{2}(\Omega)}^{2}\right), \\
& \left\|\left.\nabla \beta\right|_{L_{w}^{2}(\Omega)} \leq C|| \Pi \nabla \beta\right\|_{L_{w}^{2}(\partial \Omega)}\|\beta\|_{L_{w}^{2}(\partial \Omega)}+C(K)\left(\|\operatorname{div} \beta\|_{L_{w}^{2}(\Omega)}^{2}+\|\operatorname{curl} \beta\|_{L_{w}^{2}(\Omega)}^{2}+\|\beta\|_{L_{w}^{2}(\Omega)}^{2}\right) \text {. }
\end{aligned}
$$

Proof. Inequality (8.7) is just (A.22). Let $g^{I J}=g^{i_{1} j_{1}} \cdots g^{i_{k} j_{k}}$, (8.8) follows by induction from

$$
\begin{aligned}
\int_{\partial \Omega} g^{I J} g^{i j} \beta_{I i} \beta_{J j} w d \mu_{\gamma}= & \int_{\Omega} \nabla_{k}\left(N^{k} g^{I J}\left(N^{i} N^{j}+\gamma^{i j}\right) \beta_{I i} \beta_{J j} w\right) d \mu_{g} \\
= & \int_{\Omega}\left(\nabla_{k} N^{k}\right) g^{I J}\left(N^{i} N^{j}+\gamma^{i j}\right) \beta_{I i} \beta_{J j} w d \mu_{g}+\int_{\Omega} N^{k} g^{I J}\left(N^{i} N^{j}+\gamma^{i j}\right) \beta_{I i} \beta_{J j}\left(\nabla_{k} w\right) d \mu_{g} \\
& +2 \int_{\Omega} N^{k} g^{I J}\left(N^{i} N^{j}+\gamma^{i j}\right) \beta_{I i} \nabla_{k} \beta_{J j} w d \mu_{g}
\end{aligned}
$$

On the other hand, we have

$$
\begin{aligned}
2 \int_{\Omega} N^{k} g^{I J}\left(N^{i} N^{j}+\gamma^{i j}\right) \beta_{I i} \nabla_{k} \beta_{J j} w d \mu_{g}= & 2 \int_{\Omega} N^{k} g^{I J} N^{i} N^{j} \beta_{I i} \nabla_{k} \beta_{J j} w d \mu_{g} \\
& +2 \int_{\Omega} N^{k} g^{I J} \gamma^{i j}\left(\beta_{I i} \nabla_{k} \beta_{J j}-\beta_{I i} \nabla_{j} \beta_{J k}\right) w d \mu_{g} \\
& +2 \int_{\Omega} N^{k} g^{I J} \gamma^{i j} \beta_{I i} \nabla_{j} \beta_{J k} w d \mu_{g} .
\end{aligned}
$$

However,

$$
\begin{aligned}
2 \int_{\Omega} N^{k} g^{I J} \gamma^{i j} \beta_{I i} \nabla_{j} \beta_{J k} w d \mu_{g}= & -2 \int_{\Omega} \nabla_{j}\left(N^{k} g^{I J} \gamma^{i j}\right) \beta_{I i} \beta_{J k} w d \mu_{g} \\
& -2 \int_{\Omega} N^{k} g^{I J} \gamma^{i j} \beta_{I i} \beta_{J k} \nabla_{j} w d \mu_{g}-2 \int_{\Omega} N^{k} g^{I J} \gamma^{i j} \nabla_{j} \beta_{I i} \beta_{J k} w d \mu_{g},
\end{aligned}
$$

since $N_{j} \gamma^{i j}=0$. Hence,

$$
\begin{aligned}
\int_{\partial \Omega} g^{I J} N^{i j} \beta_{I i} \beta_{J j} w d \mu_{\gamma}= & -\int_{\partial \Omega} g^{I J} \gamma^{i j} \beta_{I i} \beta_{J j} w d \mu_{\gamma}+2 \int_{\Omega} N^{k} g^{I J} \gamma^{i j} \beta_{I i}\left(\nabla_{k} \beta_{J j}-\nabla_{j} \beta_{J k}\right) w d \mu_{g} \\
& +2 \int_{\Omega} N^{k} g^{I J} N^{i} N^{j} \beta_{I i} \nabla_{k} \beta_{J j} w d \mu_{g}-2 \int_{\Omega} N^{k} g^{I J} \gamma^{i j} \nabla_{j} \beta_{I i} \beta_{J k} d \mu_{g} \\
& -2 \int_{\Omega} \nabla_{j}\left(N^{k} g^{I J} \gamma^{i j}\right) \beta_{I i} \beta_{J k} w d \mu_{g}+\int_{\Omega}\left(\nabla_{k} N^{k}\right) g^{I J} g^{i j} \beta_{I i} \beta_{J j} w d \mu_{g} \\
& +\int_{\Omega} N^{k} g^{I J} g^{i j} \beta_{I i} \beta_{J j}\left(\nabla_{k} w\right) d \mu_{g}-2 \int_{\Omega} N^{k} g^{I J} \gamma^{i j} \beta_{I i} \beta_{J k}\left(\nabla_{j} w\right) d \mu_{g}
\end{aligned}
$$

The last four terms are bounded by $\|\left.\beta\right|_{L_{w}^{2}(\Omega)} ^{2}$ since $|\nabla N| \leq K$ and $|\nabla w| \leq C w /(1+|x|)$, whereas the terms on the first line are contributed to $\|\Pi \beta\|_{L_{w}^{2}(\partial \Omega)}^{2}$ and $\|\operatorname{curl} \beta\|_{L_{w}^{2}(\Omega)}^{2}$. Finally, the terms on the second line are contributed to $\|\operatorname{div} \beta\|_{L_{w}^{2}(\Omega)}^{2}$, and so this finishes proving (8.8). (8.9) is just (8.1) after integrating by parts. 
Theorem 8.3. (Christodoulou-Lindblad type elliptic estimates) Let $q: \Omega \rightarrow \mathbb{R}$ be a function and suppose $|\theta|+\frac{1}{l_{0}} \leq K$, we have, for any $r \geq 2$ and $\delta>0$,

$$
\begin{array}{r}
\left\|\nabla^{r} q\right\|_{L_{w}^{2}(\partial \Omega)}+\left\|\nabla^{r} q\right\|_{L_{w}^{2}(\Omega) \lesssim K} \sum_{s \leq r}\left\|\Pi \nabla^{s} q\right\|_{L_{w}^{2}(\partial \Omega)}+\sum_{s \leq r-1}\left\|\nabla^{s} \Delta q\right\|_{L_{w}^{2}(\Omega)}+\|\nabla q\|_{L_{w}^{2}(\Omega)}, \\
\left\|\nabla^{r-1} q\right\|_{L_{w}^{2}(\partial \Omega)}+\left\|\nabla^{r} q\right\|_{L_{w}^{2}(\Omega)} \lesssim K \delta \sum_{s \leq r}\left\|\Pi \nabla^{s} q\right\|_{L_{w}^{2}(\partial \Omega)}+\delta^{-1}\left(\sum_{s \leq r-2}\left\|\nabla^{s} \Delta q\right\|_{L_{w}^{2}(\Omega)}+\|\nabla q\|_{L_{w}^{2}(\Omega)}\right) .
\end{array}
$$

Proof. It suffices to prove (8.11) and (8.12) for $r=2$. By (8.9), we have

$$
\begin{aligned}
& \left\|\nabla^{2} q\right\|_{L_{w}^{2}(\Omega)} \leq C(K)\left(\left\|\Pi \nabla^{2} q\right\|_{L_{w}^{2}(\partial \Omega)}\|\nabla q\|_{L_{w}^{2}(\partial \Omega)}+\|\Delta q\|_{L_{w}^{2}(\Omega)}\right) \\
& \quad \leq \delta C(K)\left\|\Pi \nabla^{2} q\right\|_{L_{w}^{2}(\partial \Omega)}+C\left(\delta^{-1}, K\right)\|\nabla q\|_{L_{w}^{2}(\partial \Omega)}+\|\Delta q\|_{L_{w}^{2}(\Omega)} .
\end{aligned}
$$

On the other hand, by (8.8), we have

$$
\left\|\nabla^{2} q\right\|_{L_{w}^{2}(\partial \Omega)} \leq C(K)\left(\left\|\Pi \nabla^{2} q\right\|_{L_{w}^{2}(\partial \Omega)}+\|\Delta \nabla q\|_{L_{w}^{2}(\Omega)}+\|\nabla q\|_{L_{w}^{2}(\Omega)}\right) .
$$

Then (8.11) follows from (8.13)-(8.14) and induction with $\delta=1$. To prove (8.12), we have via (8.7) that

$$
\|\nabla q\|_{L_{w}^{2}(\partial \Omega)} \leq C(K)\left(\left\|\nabla^{2} q\right\|_{L_{w}^{2}(\Omega)}+\|\nabla q\|_{L_{w}^{2}(\Omega)}\right) .
$$

(8.12) then follows from (8.13) and induction.

\subsection{The weighted tensor estimate}

Theorem 8.4. Suppose that $|\theta|+\left|\frac{1}{l_{0}}\right| \leq K$, and for $q=0$ on $\partial \Omega$, then for $m=0,1$

$$
\begin{aligned}
& \left\|\Pi \nabla^{r} q\right\|_{L_{w}^{2}(\partial \Omega) \lesssim K}\left\|\left(\sum_{s \leq r-2}\left(\bar{\nabla}^{s} \theta\right)\right) \nabla_{N} q\right\|_{L_{w}^{2}(\partial \Omega)}+\sum_{l=1}^{r-1}\left\|\nabla^{r-l} q\right\|_{L_{w}^{2}(\partial \Omega)} \\
& \quad+\left(\|\theta\|_{L^{\infty}(\partial \Omega)}+\sum_{0 \leq l \leq r-2-m}\left\|\bar{\nabla}^{l} \theta\right\|_{L_{w}^{2}(\partial \Omega)}\right)\left(\sum_{0 \leq l \leq r-2+m}\left\|\nabla^{l} q\right\|_{L_{w}^{2}(\partial \Omega)}\right),
\end{aligned}
$$

where the second line drops if $0 \leq r \leq 4$.

Proof. The proof follows from the interpolation inequalities on the boundary, e.g., Theorem A.7. We refer [5] Proposition 4.7 for the detailed proof.

In addition, the weighted estimate for the second fundamental form $\theta$ is then a immediate consequence.

Theorem 8.5. Suppose that $|\theta|+\left|\frac{1}{l_{0}}\right| \leq K$, and the physical sign condition $\left|\nabla_{N} h\right| \geq \epsilon>0$ holds, then

$$
\begin{aligned}
\left\|\bar{\nabla}^{r-2} \theta\right\|_{L_{w}^{2}(\partial \Omega) \lesssim K, \frac{1}{\epsilon}}\left\|\Pi \nabla^{r} h\right\|_{L_{w}^{2}(\partial \Omega)}+\sum_{s=1}^{r-1}\left\|\nabla^{r-s} h\right\|_{L_{w}^{2}(\partial \Omega)} & \\
& +\left(\|\theta\|_{L^{\infty}(\partial \Omega)}+\sum_{s \leq r-3}\left\|\bar{\nabla}^{s} \theta\right\|_{L_{w}^{2}(\partial \Omega)}\right) \sum_{s \leq r-1}\left\|\nabla^{s} h\right\|_{L_{w}^{2}(\partial \Omega)},
\end{aligned}
$$

where the second line drops for $0 \leq r \leq 4$. 


\subsection{The weighted energy estimates for Euler equations}

The higher order weighted energies for the compressible Euler equations are

$$
E_{w, r}=\sum_{s+k=r} E_{w, s, k}+K_{w, r}+\sum_{j \leq r+1} W_{w, j}^{2}, \quad r \geq 2, \quad E_{w, r}^{*}=\sum_{r^{\prime} \leq r} E_{w, r^{\prime}},
$$

where

$$
\begin{aligned}
E_{w, s, k}(t)= & \frac{1}{2} \int_{\mathcal{D}_{t}} \rho \delta^{i j} Q\left(\partial^{s} D_{t}^{k} v_{i}, \partial^{s} D_{t}^{k} v_{j}\right) w d x+\frac{1}{2} \int_{\mathcal{D}_{t}} \rho e^{\prime}(h) Q\left(\partial^{s} D_{t}^{k} h, \partial^{s} D_{t}^{k} h\right) w d x \\
& +\frac{1}{2} \int_{\partial \mathcal{D}_{t}} \rho Q\left(\partial^{s} D_{t}^{k} h, \partial^{s} D_{t}^{k} h\right) \nu w d S,
\end{aligned}
$$

where $\nu=\left(-\nabla_{N} h\right)^{-1}$ and

$$
\begin{aligned}
K_{w, r}(t) & =\int_{\mathcal{D}_{t}} \rho\left|\partial^{r-1} \operatorname{curl} v\right|^{2} w d x, \\
W_{w, r}(t) & =\frac{1}{2}\left\|\sqrt{e^{\prime}(h)} D_{t}^{r} h\right\|_{L_{w}^{2}\left(\mathcal{D}_{t}\right)}+\frac{1}{2}\left\|\nabla D_{t}^{r-1} h\right\|_{L_{w}^{2}\left(\mathcal{D}_{t}\right)} .
\end{aligned}
$$

Using Theorem 8.3 - Theorem 8.5 the weighted Sobolev lemmas as well as the interpolation inequalities (e.g., Lemma A.4, Lemma A.5 Theorem A.6. Theorem A.7 and Theorem A.11), and the fact that our weight $w$ satisfies $\left|\partial^{r} w\right| \leq C_{r} w$, we are able to repeat the analysis we have done in Section 4 and Section 5 to obtain the weighted elliptic bounds:

$$
\begin{array}{r}
\|v\|_{w, r, 0}^{2}+\|h\|_{w, r}^{2} \leq C_{r}\left(K, M, c_{0}, E_{w, r-1}^{*}\right) E_{w, r}^{*} \\
\left\|D_{t} h\right\|_{w, r}^{2}+\langle\langle h\rangle\rangle_{w, r}^{2} \leq C_{r}\left(K, M, c_{0}, \frac{1}{\epsilon}, E_{w, r-1}^{*}\right) E_{w, r}^{*}
\end{array}
$$

where

$$
\begin{array}{r}
\|v\|_{w, r, 0}:=\sum_{k+s=r, k<r}\left\|\partial^{s} D_{t}^{k} v\right\|_{L_{w}^{2}\left(\mathcal{D}_{t}\right)}, \\
\|h\|_{w, r}:=\sum_{k+s=r, k<r}\left\|\partial^{s} D_{t}^{k} h\right\|_{L_{w}^{2}\left(\mathcal{D}_{t}\right)}+\left\|\sqrt{e^{\prime}(h)} D_{t}^{r} h\right\|_{L_{w}^{2}\left(\mathcal{D}_{t}\right)}, \\
\langle\langle h\rangle\rangle_{w, r}:=\sum_{k+s=r}\left\|\partial^{s} D_{t}^{k} h\right\|_{L_{w}^{2}\left(\partial \mathcal{D}_{t}\right)} .
\end{array}
$$

But these yield the analogous energy estimates for $E_{w, r}$.

Theorem 8.6. Let $E_{w, r}$ be defined as (8.18), then there are continuous functions $C_{r}$ such that for each fixed $r \geq 1$, we have

$$
\left|\frac{d E_{w, r}(t)}{d t}\right| \leq C_{r}\left(K, \frac{1}{\epsilon}, M, c_{0}, E_{w, r-1}^{*}\right) E_{w, r}^{*}(t),
$$

provided the (1.5) and a priori assumptions (1.24)-(1.29).

\section{A Appendix}

\section{List of notations:}

- $D_{t}$ : the material derivative

- $\partial_{i}$ : partial derivative with respect to Eulerian coordinate $x_{i}$

- $\mathcal{D}_{t} \in \mathbb{R}^{n}$ : the domain occupied by fluid particles at time $t$ in Eulerian coordinate 
- $\Omega \in \mathbb{R}^{n}$ : the domain occupied by fluid particles in Lagrangian coordinate

- $\partial_{a}=\frac{\partial}{\partial y_{a}}$ : partial derivative with respect to Lagrangian coordinate $y_{a}$

- $\nabla_{a}$ : covariant derivative with respect to $y_{a}$

- $\Pi S$ : projected tensor $S$ on the boundary

- $\bar{\nabla}, \bar{\partial}$ : projected derivative on the boundary

- $N$ : the outward unit normal of the boundary

- $\theta=\bar{\nabla} N$ : the second fundamental form of the boundary

- $\sigma=\operatorname{tr}(\theta)$ : the mean curvature

- $\kappa=\kappa(x)$ : the sound speed

- $L_{w}^{p}(\Omega)$ : The weighted $L^{p}$ space

- $W_{w}^{s, p}(\Omega)$ : The weighted Sobolev space

\section{Mixed norms}

- $\langle\langle\cdot\rangle\rangle_{r}=\sum_{k+s=r}\left\|\nabla^{s} D_{t}^{k} \cdot\right\|_{L^{2}(\partial \Omega)}$

- $\|\cdot\|_{r, 0}=\sum_{s+k=r, k<r}\left\|\nabla^{s} D_{t}^{k} \cdot\right\|_{L^{2}(\Omega)}$

- $\|\cdot\|_{r}=\|\cdot\|_{r, 0}+\left\|\sqrt{e^{\prime}(h)} D_{t}^{r} \cdot\right\|_{L^{2}(\Omega)}$

- $\|\cdot\|_{r, 1,0}=\sum_{k+s=r, k<r-1}\left\|\nabla^{s} D_{t}^{k} \cdot\right\|_{L^{2}(\Omega)}+\left\|\sqrt{e^{\prime}(h)} \nabla D_{t}^{r-1} \cdot\right\|_{L^{2}(\Omega)}$,

- $\|\cdot\|_{r, 1}=\|\cdot\|_{r, 1,0}+\left\|e^{\prime}(h) D_{t}^{r} \cdot\right\|_{L^{2}(\Omega)}$.

Weighted norms

- $\|\left. u\right|_{L_{w}^{p}(\Omega)}=\left(\int_{\Omega}|u(x)|^{p} w(x) d x\right)^{1 / p}$

- $\|u\|_{W_{w}^{s, p}(\Omega)}=\sum_{|\alpha| \leq s}\left\|\nabla^{\alpha} u\right\|_{L_{w}^{p}(\Omega)}$

\section{A.1 The geometry of the boundary, extension of normal to the interior and the geodesic normal coordinate}

The definition of our energy (1.12) relies on extending the normal to the interior, which is done by foliating the domain close to the boundary into the surface that do not self-intersect. We also want to control the time evolution of the boundary, which can be measured by the time derivative of the normal in the Lagrangian coordinate. We conclude the above statements by the following two lemmas, whose proof can be found in [5].

Lemma A.1. let $l_{0}$ be the injective radius (1.10), and let $d(y)=\operatorname{dist}_{g}(y, \partial \Omega)$ be the geodesic distance in the metric $g$ from $y$ to $\partial \Omega$. Then the co-normal $n=\nabla d$ to the set $S_{a}=\partial\{y \in \Omega: d(y)=a\}$ satisfies, when $d(y) \leq \frac{l_{0}}{2}$ that

$$
\begin{array}{r}
|\nabla n| \lesssim|\theta|_{L^{\infty}(\partial \Omega)}, \\
\left|D_{t} n\right| \lesssim\left|D_{t} g\right|_{L^{\infty}(\Omega)},
\end{array}
$$

where we have used the convention that $A \lesssim B$ means $A \leq C B$ for universal constant $C$. 
Lemma A.2. let $l_{0}$ be the injective radius (1.10), and let $d_{0}$ be a fixed number such that $\frac{l_{0}}{16} \leq d_{0} \leq \frac{l_{0}}{2}$. Let $\eta$ be a smooth cut-off function satisfying $0 \leq \eta(d) \leq 1, \eta(d)=1$ when $d \leq \frac{d_{0}}{4}$ and $\eta(d)=0$ when $d>\frac{d_{0}}{2}$. Then the psudo-Riemannian metric $\gamma$ given by

$$
\gamma_{a b}=g_{a b}-\tilde{n}_{a} \tilde{n}_{b},
$$

where $\tilde{n}_{c}=\eta\left(\frac{d}{d_{0}}\right) \nabla_{c} d$ satisfies

$$
\begin{array}{r}
|\nabla \gamma|_{L^{\infty}(\Omega)} \lesssim\left(|\theta|_{L^{\infty}(\partial \Omega)}+\frac{1}{l_{0}}\right) \\
\left|D_{t} \gamma(t, y)\right| \lesssim\left|D_{t} g\right|_{L^{\infty}(\Omega)} .
\end{array}
$$

Remark. The above two lemmas yield that the quantities $\left|D_{t} n\right|$ and $\left|D_{t} \gamma(t, y)\right|$ involved in the $Q$-inner product is controlled by the a priori assumptions (1.24)-(1.29), since $D_{t} g$ behaves like $\nabla v$ by (2.8). Hence, the time derivative on the coefficients of the $Q$-inner product generates only lower-order terms. In addition, by (1.24),$|\nabla n|$ and $|\nabla \gamma|$ are controlled by $K$, which is essential when proving the Christodoulou-Lindblad type elliptic estimates.

The next lemma introduces the partition of unity $\left\{\chi_{i}\right\}$ in a domain with sufficient regular boundary.

Lemma A.3. Suppose that $\Omega \in \mathbb{R}^{n}$ is a domain whose boundary satisfying the condition $|\theta|+\frac{1}{l_{0}} \leq K$. Then there are functions $\chi_{i} \in C_{c}^{\infty}\left(\mathbb{R}^{n}\right), i=1,2, \cdots$, such that

$$
0 \leq \chi_{i} \leq 1, \quad \sum \chi_{i}=1, \quad \sum\left|\partial^{\alpha} \chi_{i}\right| \leq C_{\alpha} K^{|\alpha|}, \quad \operatorname{diam}\left(\operatorname{supp}\left(\chi_{i}\right)\right) \leq K^{-1},
$$

and for each $x \in \mathbb{R}^{n}$, there are at most $16^{n} i$ 's such that $\chi_{i}(x) \neq 0$. Furthermore, either $\operatorname{supp}\left(\chi_{i}\right) \cup \partial \Omega$ is empty or is part of a graph contained in $\partial \Omega$, for which (possibly after a rotation) is given by

$$
x_{n}=f_{i}\left(x^{\prime}\right), \quad\left|\partial f_{i}\right| \leq c_{1}, \quad N\left(x_{i}\right)=e_{n}, \quad \text { for }\left|x^{\prime}-x_{i}^{\prime}\right| \leq l_{0} .
$$

Proof. See [5].

\section{A.2 Sobolev lemmas}

Let us now state some Sobolev lemmas in a domain with boundary, whose proofs are standard and can be found in [5], 11] and [25].

Lemma A.4. (Interior Sobolev inequalities) Suppose $\frac{1}{l_{0}} \leq K$ and $\alpha$ is a $(0, r)$ tensor, then

$$
\begin{aligned}
\|\alpha\|_{L^{\frac{2 n}{n-2 s}(\Omega)}} & \lesssim K \sum_{l=0}^{s}\left\|\nabla^{l} \alpha\right\|_{L^{2}(\Omega)}, \quad 2 s<n, \\
\|\alpha\|_{L^{\infty}(\Omega)} & \lesssim K \sum_{l=0}^{s}\left\|\nabla^{l} \alpha\right\|_{L^{2}(\Omega)}, \quad 2 s>n .
\end{aligned}
$$

These inequalities remains valid in weighted spaces $L_{w}^{p}(\Omega)$ if the weight satisfies $\left|\partial^{r} w\right| \leq C_{r} w /(1+|x|)^{r}$.

Proof. See [5].

Similarly, on the boundary $\partial \Omega$, we have

Lemma A.5. (Boundary Sobolev inequalities)

$$
\begin{aligned}
&\|\alpha\|_{L^{\frac{2(n-1)}{n-1-2 s}(\Omega)}} \lesssim_{K} \sum_{l=0}^{s}\left\|\nabla^{l} \alpha\right\|_{L^{2}(\partial \Omega)}, 2 s<n-1, \\
&\|\alpha\|_{L^{\infty}(\Omega) \lesssim K} \delta\left\|\nabla^{s} \alpha\right\|_{L^{2}(\partial \Omega)}+\delta^{-1} \sum_{l=0}^{s-1}\left\|\nabla^{l} \alpha\right\|_{L^{2}(\partial \Omega)}, \quad 2 s>n-1,
\end{aligned}
$$

for any $\delta>0$. These inequalities remain valid in weighted spaces $L_{w}^{p}(\Omega)$ as well. In addition, for the boundary we can also interpret the norm be given by the inner product $\langle\alpha, \alpha\rangle=\gamma^{I J} \alpha_{I} \alpha_{J}$, and the covariant derivative is then given by $\bar{\nabla}$. 


\section{A.3 Interpolation on spatial derivatives}

We shall first record spatial interpolation inequalities. Most of the results are are standard in $\mathbb{R}^{n}$, but we must control how it depends on the geometry of our evolving domain. The coefficients involved in our inequalities depend on $K$, whose reciprocal is the lower bound for the injective radius $l_{0}$.

Theorem A.6. (Interior interpolation) Let $u$ be a $(0, s)$ tensor, and suppose $\frac{1}{l_{0}} \leq K$, we have

$$
\sum_{j=0}^{l}\left\|\nabla^{j} u\right\|_{L^{\frac{2 r}{k}(\Omega)}} \lesssim\|u\|_{L^{\frac{2(r-l)}{k-l}}(\Omega)}^{1-\frac{l}{r}}\left(\sum_{i=0}^{r}\left\|\nabla^{i} u\right\|_{L^{2}(\Omega)} K^{r-i}\right)^{\frac{l}{r}} .
$$

In particular, if $k=l$,

$$
\sum_{j=0}^{k}\left\|\nabla^{j} u\right\|_{L^{\frac{2 r}{k}(\Omega)}} \lesssim\|u\|_{L^{\infty}(\Omega)}^{1-\frac{k}{r}}\left(\sum_{i=0}^{r}\left\|\nabla^{i} u\right\|_{L^{2}(\Omega)} K^{r-i}\right)^{\frac{k}{r}} .
$$

These inequalities remains valid when $L^{p}(\Omega)$ is replaced by $L_{w}^{p}(\Omega)$ if $w \geq 0$ satisfies $\left|\partial^{r} w\right| \leq C_{r} w /(1+|x|)^{r}$.

Proof. It suffices to prove (A.11) with $s=0$, i.e., when $u$ is a function, since $u$ can be replaced by its magnitude $|u|$. Furthermore, since (A.11) is equivalent to

$$
\sum_{j \leq l}\left\|\nabla^{j} u\right\|_{L^{s}(\Omega)} \leq C(K)\|u\|_{L^{q}(\Omega)}^{1-a}\left(\sum_{i \leq r}\left\|\nabla^{i} u\right\|_{L^{p}(\Omega)}\right)^{a}
$$

where $a=l / r$ and $\frac{r}{s}=\frac{l}{p}+\frac{r-l}{q}$. We can further reduce (A.13) to the case when $r=2$ and $s=1$, because the general cases follow from the logarithmic convexity.

Using the partition of unity $\left\{\chi_{i}\right\}$ defined in Lemma A.3, we write $u=\sum u_{i}$, where $u_{i}=\chi_{i} u$. In a neighbourhood of $\operatorname{supp}\left(\chi_{i}\right)$, we can then write $\Omega$ as a graph after a rotation:

$$
x_{n}=f\left(x^{\prime}\right), \quad|\partial f| \leq C .
$$

We now define the reflection

$$
\tilde{u}_{i}(x)= \begin{cases}u_{i}(x), & \text { when } x \in \Omega \\ u_{i}(\tilde{x}), & \text { when } x \in \Omega^{c}\end{cases}
$$

Here, $\tilde{x}=\left(x^{\prime}, x_{n}-2\left(x_{n}-f\left(x^{\prime}\right)\right)\right.$. Then by the interpolation in $\mathbb{R}^{n}$, we have

$$
\left\|\nabla \tilde{u}_{i}\right\|_{L^{s}\left(\mathbb{R}^{n}\right)}^{2} \leq\left\|\tilde{u}_{i}\right\|_{L^{q}\left(\mathbb{R}^{n}\right)}\left\|\nabla^{2} \tilde{u}_{i}\right\|_{L^{p}\left(\mathbb{R}^{n}\right)} .
$$

But since for every $1 \leq p^{\prime} \leq \infty$ and $\left|\partial \tilde{x}^{i} / \partial x^{j}\right| \leq C$,

$$
\left\|\nabla^{\alpha} \tilde{u}_{i}\right\|_{L^{p^{\prime}\left(\mathbb{R}^{n}\right)}} \leq C\left(\left\|\nabla^{\alpha} u_{i}\right\|_{L^{p^{\prime}}(\Omega)}+\left\|\nabla^{\alpha} \tilde{u}_{i}\right\|_{L^{p^{\prime}}\left(\Omega^{c}\right)}\right) \leq C\left\|\nabla^{\alpha} u_{i}\right\|_{L^{p^{\prime}}(\Omega)},
$$

for $|\alpha| \leq 2$. Furthermore, we have

$$
\begin{array}{r}
\left\|\nabla u_{i}\right\|_{L^{p^{\prime}}(\Omega)} \leq C\left\|\left(\nabla \chi_{i}\right) u\right\|_{L^{p^{\prime}}(\Omega)}+C\left\|\chi_{i} \nabla u\right\|_{L^{p^{\prime}}(\Omega)}, \\
\left\|\nabla^{2} u_{i}\right\|_{L^{p^{\prime}}(\Omega)} \leq C\left\|\left(\nabla^{2} \chi_{i}\right) u\right\|_{L^{p^{\prime}}(\Omega)}+C\left\|\left(\nabla \chi_{i}\right) \nabla u\right\|_{L^{p^{\prime}}(\Omega)}+C\left\|\chi_{i} \nabla^{2} u\right\|_{L^{p^{\prime}}(\Omega)}
\end{array}
$$

and this gives A.13) via Lemma A.3 for $l=1$ and $r=2$. The general case follows by letting $M_{k}=$ $\sum_{i \leq k}\left\|\nabla^{i} u\right\|_{L^{s(k)}}$, and so far we have proven $M_{1} \lesssim M_{0} M_{2}$, and hence we get $M_{k}^{2} \lesssim M_{k-1} M_{k+1}$ follows from this special case. But the logarithmic convexity then gives $M_{k} \lesssim M_{0}^{(r-l) / r} M_{r}^{l / r}$. Finally, the weighted case follow from the non-weighted case since $\left|\partial^{r} w\right| \lesssim|w| /(1+|x|)^{r}$. 


\section{A.4 Interpolation on $\partial \Omega$}

Theorem A.7. (Boundary interpolation) Let $u$ be a $(0, s)$ tensor, then

$$
\left\|\bar{\nabla}^{l} u\right\|_{L^{\frac{2 r}{k}}(\partial \Omega)} \lesssim\|u\|_{L^{\frac{2(r-l)}{k-l}}(\partial \Omega)}^{1-\frac{l}{r}}\left\|\bar{\nabla}^{r} u\right\|_{L^{2}(\partial \Omega)}^{\frac{l}{r}} .
$$

In particular, if $k=l$,

$$
\left\|\bar{\nabla}^{k} u\right\|_{L^{\frac{2 r}{k}(\partial \Omega)}} \lesssim\|u\|_{L^{\infty}(\partial \Omega)}^{1-\frac{k}{r}}\left\|\bar{\nabla}^{r} u\right\|_{L^{2}(\partial \Omega)}^{\frac{k}{r}} .
$$

Furthermore, if $w \geq 0$ satisfies $\left|\partial^{r} w\right| \leq C_{r} w /(1+|x|)^{r}$, then

$$
\left\|\bar{\nabla}^{l} u\right\|_{L^{\frac{2 r}{k} w(\partial \Omega)}} \lesssim\|u\|_{L^{\frac{2(r-l)}{k-l} w_{(\partial \Omega)}}}^{1-\frac{l}{r}}\left(\sum_{i \leq r}\left\|\bar{\nabla}^{i} u\right\|_{L_{w}^{2}(\partial \Omega)}^{\frac{l}{r}}\right) .
$$

Proof. The proof for (A.14) can be found in [5], and (A.16) follows from the same proof and the lower order terms on the RHS is generated when the derivatives fall on the weight function $w$.

\section{A.5 Elliptic estimates in weighted Sobolev spaces}

This section is devoted to set up the elliptic estimates in weighted Sobolev spaces $H_{w}^{s}(\Omega)$ (Definition 7.1) with weight $w(x)=\left(1+|x|^{2}\right)^{\mu}, \mu \geq 2$, where $\Omega \subset \mathbb{R}^{n}, n=2,3$ be a smooth domain, diffeomorphic to the half space $\left\{x \in \mathbb{R}^{n}: x_{n} \leq 0\right\}$. Consider the Dirichlet boundary value problem

$$
\left\{\begin{array}{l}
\Delta u=f, \quad \text { in } \Omega \\
u=0, \quad \text { in } \partial \Omega
\end{array}\right.
$$

then the following $L^{2}$ elliptic estimate holds.

Theorem A.8. (Boccia-Salvato-Transirico [2]) Fix $s \geq 2$ and $p \in(0, \infty)$, then

$$
\|u\|_{W_{w}^{s, p}(\Omega)} \leq C\left(\|f\|_{W_{w}^{s-2, p}(\Omega)}+\|u\|_{L_{w}^{p}(\Omega)}\right)
$$

holds for all $u \in W_{w}^{s, p}(\Omega)$ that solves A.17).

Now we show that the $\|u\|_{L_{w}^{p}(\Omega)}$ on the RHS of (A.18) can in fact be dropped. It is worth to mention here that we have no problem to drop this term if $\Omega$ were bounded, since $\lambda=0$ is not an eigenvalue of $\Delta$ in this case (e.g, chapter 6.2 in Evans [11]). However, it is in general impossible to drop the term $\|u\|_{L^{2}}$ in elliptic estimates when $\Omega$ is unbounded, unless $u$ is sufficiently smooth and decays fast enough at infinity.

Theorem A.9. (Rellich-Kondrachov embedding for weighted spaces) The spaces $H_{0, w}^{1}(\Omega)$ (the space consists of $u \in H_{w}^{1}(\Omega)$ with $\left.\left.u\right|_{\partial \Omega}=0\right)$ are compactly embedding in the spaces $L^{q}(\Omega)$ for any $q<2 n /(n-2)$.

Proof. We follow the proof given by Gilbarg-Trudinger [13] with some modifications. We initially assume $q=1$. Let $\mathcal{A}$ be a bounded subset in $H_{0, w}^{1}(\Omega)$. Without loss of generality we assume that $\mathcal{A} \in C_{c}^{1}(\Omega)$ and that $\|u\|_{H_{w}^{1}(\Omega)} \leq 1$. For fixed $\delta>0$, let $\mathcal{A}_{\delta}:=\left\{u_{\delta}: u \in \mathcal{A}\right\}$, where $u_{\delta}$ is the mollification of $u$, i.e., $u_{\delta}=\eta_{\delta} * u$, where $\eta(x)$ is a smooth bump function supported in the unit ball satisfying $\int \eta(x) d x=1$, and $\eta_{\delta}=\delta^{-n} \eta\left(\delta^{-1} x\right)$.

For each $u \in \mathcal{A}$, we have

$$
\begin{array}{r}
\left\|u_{\delta}(x)\right\|_{L^{\infty}(\Omega)} \leq \delta^{-n}\|\eta\|_{L^{\infty}(\Omega)}\|u\|_{H_{w}^{1}(\Omega)}, \\
\left\|\nabla u_{\delta}(x)\right\|_{L^{\infty}(\Omega)} \leq \delta^{-n-1}\|\nabla \eta\|_{L^{\infty}(\Omega)}\|u\|_{H_{w}^{1}(\Omega)},
\end{array}
$$

and so $\mathcal{A}_{\delta}$ is a bounded, equicontinuous subset of $C_{c}(\Omega)$ and hence precompact in $C_{c}(\Omega)$, and consequently also precompact in $L^{1}(\Omega)$. Nevertheless, we have 


$$
\begin{aligned}
& \left|u(x)-u_{\delta}(x)\right| \leq \int_{|z| \leq 1} \eta(z) \mid u(x)-u(x-\delta z) d z \\
& \quad \leq \int_{|z| \leq 1} \eta(z) \int_{0}^{\delta|z|} \mid \nabla_{r} u\left(x-r \frac{z}{|z|}\right) d r d z
\end{aligned}
$$

and hence

$$
\begin{aligned}
& \int_{\Omega}\left|u(x)-u_{\delta}(x)\right| d x \leq \delta \int_{\Omega}|\nabla u| d x \\
& \quad \leq \delta\left(\int_{\mathbb{R}^{n}} \frac{1}{\left(1+|x|^{2}\right)^{\mu}} d x\right)^{1 / 2}\|u\|_{H_{w}^{1}(\Omega)} .
\end{aligned}
$$

But since $\int_{\mathbb{R}^{n}} \frac{1}{\left(1+|x|^{2}\right)^{\mu}} d x<\infty$ when $n \leq 3$ and so $u_{\delta}$ is uniformly close to $u$ in $L^{1}(\Omega)$. It then follows that $\mathcal{A}$ is precompact in $L^{1}(\Omega)$. Now, for any $q<2 n /(n-2)$, we have

$$
\|u\|_{L^{q}(\Omega)} \lesssim\|u\|_{L^{1}}^{a}\|u\|_{L^{2 n /(n-2)}}^{1-a}
$$

for some $0<a<1$ via interpolation. In addition, we have

$$
\|u\|_{L^{2 n /(n-2)}} \lesssim\|u\|_{H_{w}^{1}}
$$

by Sobolev lemma and the fact that $w(x) \geq 1$. This concludes that a bounded set in $H_{0, w}^{1}(\Omega)$ must be precompact in $L^{q}(\Omega)$.

Remark. The classical Rellich-Kondrachov embedding theorem yields that $H^{1}(\Omega)$ is compactly embedding in the spaces $L^{q}(\Omega)$ when $\Omega$ is bounded.

Theorem A.10. (Improved elliptic estimates) Let $u \in H_{w}^{s}(\Omega) \cap H_{0, w}^{1}(\Omega)$ be a function that solves (A.17), and if $f \in H_{w}^{s-2}(\Omega)$ then

$$
\|u\|_{H_{w}^{s}(\Omega)} \leq C\|f\|_{H_{w}^{s-2}(\Omega)} .
$$

Proof. It suffices to prove (A.19) when $s=2$. If (A.19) is not true, then there exists a sequence $\left\{u_{m}\right\} \subset$ $H_{w}^{2}(\Omega) \cap H_{0, w}^{1}(\Omega)$ satisfying

$$
\left\|u_{m}\right\|_{L_{w}^{2}(\Omega)}=1, \quad\left\|u_{m}\right\|_{L^{2}(\Omega)} \leq 1, \quad\left\|\Delta u_{m}\right\|_{L_{w}^{2}(\Omega)} \rightarrow 0 .
$$

By virtue of the apriori estimate (A.18), Theorem A.9 and the weakly compactness of bounded subsets in $H_{w}^{2}(\Omega)$, there exists a subsequence, relabelled as $\left\{u_{m}\right\}$, converging weakly to a function $u \in H_{w}^{2}(\Omega) \cap H_{0, w}^{1}(\Omega)$ satisfying $\|u\|_{L_{w}^{2}(\Omega)}=1$. However, for any $\phi \in L_{w}^{2}(\Omega)$, we must have

$$
\int_{\Omega} \phi(\Delta u) w=0
$$

Hence, $\Delta u=0$ and so $u=0$ by the uniqueness assertion (e.g. G-T [13], Theorem 8.9 or maximum principle since $u$ decays to 0 at $\infty)$. But this implies $\|u\|_{L_{w}^{2}}=0$, a contradiction.

\section{A.6 Gagliardo-Nirenberg interpolation inequality}

Theorem A.11. Let $u$ be a $(0, r)$ tensor defined on $\partial \Omega \in \mathbb{R}^{2}$ and suppose $\frac{1}{l_{0}} \leq K$, we have

$$
\|u\|_{L^{4}(\partial \Omega)}^{2} \lesssim K|| u\left\|_{L^{2}(\partial \Omega)}\right\| u \|_{H^{1}(\partial \Omega)},
$$

where $H^{1}(\partial \Omega)$ is defined via tangential derivative $\bar{\nabla}$. Furthermore, (A.20) remains valid in the case of weighted Sobolev spaces. 
Proof. It suffices for us to work in the local coordinate charts $\left\{U_{i}\right\}$ of $\partial \Omega$. We consider the corresponding partition of unity $\left\{\chi_{i}\right\}$, where each $\chi_{i}$ is supported in $U_{i}$ and vanishing on the boundary of $U_{i}$. As proved in Lemma A.3. $\chi_{i}$ can be chosen to satisify

$$
\sum_{i}\left|\bar{\nabla} \chi_{i}\right| \leq C(K)
$$

Now by the result of Constantin and Seregin [6], we have

$$
\left\|u_{i}\right\|_{L^{4}\left(U_{i}\right)}^{2} \lesssim\left\|u_{i}\right\|_{L^{2}\left(U_{i}\right)}|| \bar{\nabla} u_{i} \|_{L^{2}\left(U_{i}\right)}
$$

where $u_{i}=\chi_{i} u$. But since

$$
\left\|\bar{\nabla} u_{i}\right\|_{L^{2}\left(U_{i}\right)}=\left\|\bar{\nabla}\left(\chi_{i} u\right)\right\|_{L^{2}\left(U_{i}\right)} \leq\left|\bar{\nabla} \chi_{i}\right|_{L^{\infty}}\|u\|_{L^{2}\left(U_{i}\right)}+\left\|\chi_{i} \bar{\nabla} u\right\|_{L^{2}\left(U_{i}\right)} .
$$

Hence, (A.20) follows by summing up (A.11). This proof remains valid with $L^{p}$ being replaced by $L_{w}^{p}$, where $w$ is defined in Section A.5

\section{A.7 The trace theorem}

Theorem A.12. (The trace theorem) Let $\alpha$ be a $(0, r)$ tensor, and assume that $|\theta|_{L^{\infty}(\partial \Omega)}+\frac{1}{l_{0}} \leq K$, then

$$
\|\alpha\|_{L^{2}(\partial \Omega)} \lesssim K \sum_{j \leq 1}\left\|\nabla^{j} \alpha\right\|_{L^{2}(\Omega)}
$$

Furthermore,

$$
\|\alpha\|_{L_{w}^{2}(\partial \Omega)} \lesssim K \sum_{j \leq 1}\left\|\nabla^{j} \alpha\right\|_{L_{w}^{2}(\Omega)}
$$

Here, $w$ is defined in Section A.5

Proof. It suffices to show (A.22) only, since the proof for A.21) is almost identical. Let $N$ be the extension of the normal in the interior of $\Omega$ given by the geodesic normal coordinate (i.e., Lemma A.1). Then

$$
\int_{\partial \Omega}|\alpha|^{2} w d \mu_{\gamma}=\int_{\Omega} \nabla_{k}\left(N^{k}|\alpha|^{2} w\right) d \mu_{g} .
$$

But since $|\nabla N| \leq K$ and $|\nabla w| \leq C w,($ A.22) follows.

Acknowegement I would like to express my deepest thanks to my advisor Hans Lindblad for many useful suggestions and comments. I would like to thank Marcelo Disconzi, Theo Drivas, Dan Ginsberg, Chris Kauffman, Yannick Sire, Qingtang Su, Shengwen Wang, Yi Wang, Yakun Xi and Hang Xu for many long and insightful discussions. In addition, I thank the anonymous referee for careful reading and helpful comments.

\section{References}

[1] Alazard, T. Incompressible limit of the nonisentropic Euler equations with the solid wall boundary conditions. Adv. Differential Equations, 10(1): 19-44, 2005.

[2] Boccia, S.; Salvato, M.; Transirico, M. A priori bounds for elliptic operators in weighted Sobolev spaces. J. Math. Inequal. 6, no. 2, 307-318, 2012.

[3] Cheng, B. Low-Mach-number Euler equations with solid-wall boundary condition and general initial data. arXiv:1006.1148, 2010. 
[4] Cheng, B. Improved accuracy of incompressible approximation of compressible Euler equations. SIAM J. Math. Anal., 46(6), 3838-3864, 2014.

[5] Christodoulou, D.; Lindblad, H. On the motion of the free surface of a liquid. Comm. Pure Appl. Math., 53(12): 1536-1602, 2000.

[6] Constantin, P.; Seregin, G. Hölder continuity of solutions of 2D Navier-Stokes equations with singular forcing. Nonlinear partial differential equations and related topics, 87-95. Amer. Math. Soc. Transl. Ser. 2, 229, Adv. Math. Sci., 64, Amer. Math. Soc., Providence, RI, 2010.

[7] Disconzi, M. M.; Ebin, D. G. Motion of slightly compressible fluids in a bounded domain, II. Commun. Contemp. Mathematics., 19(04): 1650054, 2017.

[8] Ebin, D. G. The motion of slightly compressible fluids viewed as a motion with strong constraining force. Ann. of Math., 141-200, 1977.

[9] Ebin, D. G. Motion of slightly compressible fluids in a bounded domain. I. Comm. Pure Appl. Math. 35(4): 451-485, 1982.

[10] Ebin, D. G. The equations of motion of a perfect fluid with free boundary are not well posed. Communications in Partial Differential Equations, 12(10): 1175-1201, 1987.

[11] Evans, L.C. Partial differential equations. Second edition. Graduate Studies in Mathematics, 19. American Mathematical Society, Providence, RI, 2010.

[12] Germain, P.; Masmoudi, N.; Shatah, J. Global solutions for the gravity water waves equation in dimension 3. Ann. of Math. (2) 175, no. 2, 691-754, 2012.

[13] Gilbarg, D.; Trudinger, N.S. Elliptic partial differential equations of second order. Reprint of the 1998 edition. Classics in Mathematics. Springer-Verlag, Berlin, 2001.

[14] Hunter, J.; Ifrim, M.; Tataru, D. Two dimensional water waves in holomorphic coordinates. Comm. Math. Phys. 346, no. 2, 483-552, 2016.

[15] Ifrim, M.; Tataru, D. Two dimensional water waves in holomorphic coordinates II: global solutions. Bull. Soc. Math. France 144, no. 2, 369-394, 2016.

[16] Ionescu, A.D.; Pusateri, F. Global solutions for the gravity water waves system in 2d. Invent. math. 199, no. 3, 653-804, 2011.

[17] Klainerman, S.; Majda, A. Singular limits of quasilinear hyperbolic systems with large parameters and the incompressible limit of compressible fluids. Comm. Pure Appl Math., 34(4): 481-524,1981.

[18] Klainerman, S.; Majda, A. Compressible and incompressible fluids. Comm. Pure Appl Math., 35(5): 629-651, 1982.

[19] Lindblad, H. Well posedness for the motion of a compressible liquid with free surface boundary. Comm. Math. Phys. 260 , no. 2, 319-392, 2005.

[20] Lindblad, H.; Luo, C. A priori estimates for the compressible Euler equations for a liquid with free surface boundary and the incompressible limit. Comm. Pure Appl. Math., 2018.

[21] Majda, A. Compressible fluid flow and systems of conservation laws in several space variables. Vol. 53. Springer Science \& Business Media, 2012.

[22] Métivier, G.; Schochet, S. The incompressible limit of the non-isentropic Euler equations. Arch. Ration. Mech. Anal., 158(1): 61-90, 2001.

[23] Schochet, S. The compressible Euler equations in a bounded domain: existence of solutions and the incompressible limit. Comm. Math. Phys., 104(1): 49-75, 1986. 
[24] Trakhinin, Y. Local existence for the free boundary problem for nonrelativistic and relativistic compressible Euler equations with a vacuum boundary condition. Comm Pure Appl. Math., 62(11): 1551-1594, 2009.

[25] Turesson, B.O. Nonlinear potential theory and weighted Sobolev spaces. Lecture Notes in Mathematics, 1736. Springer-Verlag, Berlin, 2000.

[26] Wu, S. Well-posedness in Sobolev spaces of the full water wave problem in 2-D. Invent. Math. 130, no. 1, 39-72, 1997.

[27] Wu, S. Well-posedness in Sobolev spaces of the full water wave problem in 3-D. J. Amer. Math. Soc. 12, no. 2, 445-495, 1999.

[28] Wu, S. Almost global wellposedness of the 2-D full water wave problem. Invent. Math. 177, no. 1, 45-135, 2009.

[29] Wu, S. Global wellposedness of the 3-D full water wave problem. Invent. Math. 184, no. 1, 125-220, 2011.

[30] Zhang, P.; Zhang, Z. On the free boundary problem of threedimensional incompressible Euler equations. Comm. Pure Appl. Math. 61, no. 7, 877-940, 2008. 